\title{
Search for long-lived particles that decay into final states containing two electrons or two muons in proton-proton collisions at $\sqrt{s}=8 \mathrm{TeV}$
}

\author{
V. Khachatryan et al. \\ (CMS Collaboration)
}

(Received 25 November 2014; published 18 March 2015)

\begin{abstract}
A search is performed for long-lived particles that decay into final states that include a pair of electrons or a pair of muons. The experimental signature is a distinctive topology consisting of a pair of charged leptons originating from a displaced secondary vertex. Events corresponding to an integrated luminosity of $19.6(20.5) \mathrm{fb}^{-1}$ in the electron (muon) channel were collected with the CMS detector at the CERN LHC in proton-proton collisions at $\sqrt{s}=8 \mathrm{TeV}$. No significant excess is observed above standard model expectations. Upper limits on the product of the cross section and branching fraction of such a signal are presented as a function of the long-lived particle's mean proper decay length. The limits are presented in an approximately model-independent way, allowing them to be applied to a wide class of models yielding the above topology. Over much of the investigated parameter space, the limits obtained are the most stringent to date. In the specific case of a model in which a Higgs boson in the mass range $125-1000 \mathrm{GeV} / c^{2}$ decays into a pair of long-lived neutral bosons in the mass range $20-350 \mathrm{GeV} / c^{2}$, each of which can then decay to dileptons, the upper limits obtained are typically in the range $0.2-10 \mathrm{fb}$ for mean proper decay lengths of the long-lived particles in the range $0.01-100 \mathrm{~cm}$. In the case of the lowest Higgs mass considered $\left(125 \mathrm{GeV} / \mathrm{c}^{2}\right)$, the limits are in the range 2-50 fb. These limits are sensitive to Higgs boson branching fractions as low as $10^{-4}$.
\end{abstract}

DOI: $10.1103 /$ PhysRevD.91.052012

PACS numbers: 14.80.Ec, 12.60.-i, 13.85.Rm

\section{INTRODUCTION}

Long-lived particles, which could manifest themselves through their delayed decays to leptons, are predicted in many extensions of the standard model (SM). For example, such particles could occur in supersymmetric (SUSY) scenarios such as "split SUSY" [1] or SUSY with very weak R-parity violation [2], "hidden valley" models [3], and the "minimal $B-L$ extension of the standard model [4]."

In this paper we present an inclusive search for massive, long-lived exotic particles that decay to final states that include a pair of charged leptons using proton-proton $(p p)$ collision data collected at $\sqrt{s}=8 \mathrm{TeV}$ during 2012 with the compact muon solenoid (CMS) detector at the CERN LHC. Specifically, we search for events containing a pair of electrons or muons (dileptons) originating from a common secondary vertex within the volume of the CMS tracker, and with a significant transverse displacement from the event primary vertex. This topological signature has the potential to provide clear evidence for physics beyond

* Full author list given at the end of the article.

Published by the American Physical Society under the terms of the Creative Commons Attribution 3.0 License. Further distribution of this work must maintain attribution to the author(s) and the published articles title, journal citation, and DOI. the SM. Furthermore, it is almost free of background from SM processes.

The search results are formally obtained within the context of two specific models; however, they are presented in an approximately model-independent way, allowing them to be applied to a wide range of models in which long-lived particles decay to final states that include dileptons. In the first model, the long-lived particle is a spinless boson $X$, which has a nonzero branching fraction to dileptons. The $X$ is pair-produced in the decay of a nonSM Higgs boson, $H \rightarrow X X, X \rightarrow \ell^{+} \ell^{-}$[5], where the Higgs boson is produced through gluon-gluon fusion and $\ell$ represents either an electron or a muon. In the second model, the long-lived particle is a neutralino $\tilde{\chi}^{0}$ which can decay via R-parity violating couplings into a neutrino and two charged leptons $[2,6]$. The neutralino is produced in events containing a pair of squarks, where a squark can decay via the process $\tilde{q} \rightarrow q \tilde{\chi}^{0}, \tilde{\chi}^{0} \rightarrow \ell^{+} \ell^{-} \nu$. Both models predict up to two displaced dilepton vertices per event in the CMS tracker volume, of which we only require one to be found. In this paper, we will use "LL particle" to refer to any long-lived particle, such as the $X$ or $\tilde{\chi}^{0}$ particle considered in our signal models.

The search presented here is an update of a previous CMS analysis that used a smaller data sample collected at $\sqrt{s}=7 \mathrm{TeV}$ [7] in 2011. Improvements to the previous search include the higher integrated luminosity collected in 2012, which increases the sensitivity of the search, and an 
improved analysis strategy, which substantially broadens the range of signal models to which the analysis is sensitive. The analysis complements two recent CMS publications: one searching for events that contain one electron and one muon from LL particle decays [8], and another that searches for LL particles decaying to dijets [9].

The D0 Collaboration has published the results of a search for leptons from nonprompt decays in its tracker volume [10,11], performed at $\sqrt{s}=1.96 \mathrm{TeV}$ at the Fermilab Tevatron. The ATLAS Collaboration has also performed related searches for long-lived particles using different decay channels [12,13], or lower-mass LL particles [14], compared to those considered in this paper.

\section{CMS DETECTOR}

The central feature of the CMS apparatus is a superconducting solenoid of $6 \mathrm{~m}$ internal diameter providing an axial field of 3.8 T. Within the field volume are a silicon pixel and strip tracker, a lead tungstate crystal electromagnetic calorimeter (ECAL), and a brass and scintillator hadron calorimeter. Muons are identified in gas-ionization detectors embedded in the steel flux-return yoke of the solenoid. A detailed description of the complete CMS detector, together with a definition of the coordinate system used and the relevant kinematic variables, can be found in Ref. [15].

The silicon tracker is composed of pixel detectors (three barrel layers, and two forward disks at both ends of the detector) surrounded by strip detectors (ten barrel layers, and three inner disks and nine forward disks at both ends of the detector). The tracker covers the pseudorapidity range $|\eta|<2.5$. The pixel tracker and a subset of the strip tracker layers provide three-dimensional measurements of hit positions. The other strip tracker layers measure hit position only in $(r, \phi)$ in the barrel, or $(z, \phi)$ in the end cap. Taking advantage of the strong magnetic field and the high granularity of the silicon tracker, promptly produced charged particles with transverse momentum $p_{\mathrm{T}}=100 \mathrm{GeV} / c$ are reconstructed with a resolution of $\approx 1.5 \%$ in $p_{\mathrm{T}}$ and of $\approx 15 \mu \mathrm{m}$ in transverse impact parameter $d_{0}$. The track reconstruction algorithms [16] are able to reconstruct displaced tracks with transverse impact parameters up to $\approx 25 \mathrm{~cm}$ produced by particles decaying up to $\approx 50 \mathrm{~cm}$ from the beam line. The performance of the track reconstruction algorithms has been studied with simulated events [16] and data [17]. The silicon tracker is also used to reconstruct the primary vertex position with a precision of $10-12 \mu \mathrm{m}$ in each dimension.

The ECAL consists of nearly 76000 lead tungstate crystals, which provide coverage for $|\eta|<3$. Its relative energy resolution improves with increasing energy. For energy deposits in the ECAL produced by electrons or photons of $E_{\mathrm{T}} \approx 60 \mathrm{GeV}$, where $E_{\mathrm{T}}=E \sin (\theta)$, the resolution varies between $1.1 \%$ and $5 \%$ depending on their pseudorapidity [18]. Muons are measured in the range $|\eta|<2.4$ using detection planes based on three technologies: drift tubes in the barrel region, cathode strip chambers in the end caps, and resistive-plate chambers in the barrel and end caps.

The first level of the CMS trigger system, composed of custom hardware processors, selects events of interest using information from the calorimeters and the muon detectors. A high-level trigger processor farm then employs the full event information to further decrease the event rate.

\section{DATA AND SIMULATED SAMPLES}

Data from $p p$ collisions at $\sqrt{s}=8 \mathrm{TeV}$, corresponding to an integrated luminosity of $19.6 \pm 0.5$ (20.5 \pm $0.5) \mathrm{fb}^{-1}$, are used for the search in the electron (muon) channel. The lower effective luminosity in the electron channel is due to different data quality requirements for the relevant subdetectors compared to those in the muon channel.

The electron channel data are collected with a high-level trigger [19] that requires two clustered energy deposits in the ECAL. The leading (subleading) energy deposit is required to have transverse energy $E_{\mathrm{T}}>36(22) \mathrm{GeV}$, and both clusters are required to pass loose requirements on their compatibility with a photon/electron hypothesis. The muon channel trigger requires two muons, each reconstructed in the muon detectors without using any primary vertex constraint and having $p_{\mathrm{T}}>23 \mathrm{GeV} / c$. To suppress muons from cosmic rays, the three-dimensional opening angle between the two muons must be less than 2.5 radians. Tracker information is not used in either trigger, as the track reconstruction algorithm used in the high-level trigger (as opposed to the standard offline track reconstruction) is not designed for finding displaced tracks.

For the $H \rightarrow X X$ model, simulated signal samples are generated using PYTHIA v6.426 [20] to simulate $H$ production through gluon-gluon fusion $(g g \rightarrow H)$. Subsequently, the $H$ is forced to decay into $X X$, with the $X$ bosons each decaying to dileptons $\left(X \rightarrow \ell^{+} \ell^{-}\right)$. Several samples are generated with different combinations of the mass of the $H\left(m_{H}=125,200,400,1000 \mathrm{GeV} / c^{2}\right)$ and the mass of the $X$ boson $\left(m_{X}=20,50,150\right.$, $350 \mathrm{GeV} / c^{2}$ ). The Higgs boson resonance is assumed to be narrow for the purposes of simulation, but the impact of this assumption on the analysis is negligible. Furthermore, each sample is produced with three different $X$ boson lifetimes corresponding to mean transverse decay lengths of approximately 2,20 , and $200 \mathrm{~cm}$ in the laboratory frame. For the $\tilde{\chi}^{0} \rightarrow \ell^{+} \ell^{-} \nu$ model, PYTHIA is used to simulate squark pair production and subsequent decay to $\tilde{\chi}^{0}$, using four combinations of squark and neutralino masses $\left(m_{\tilde{q}}, m_{\tilde{\chi}^{0}}\right)=(1500,494),(1000,148),(350,148)$, and $(120,48) \mathrm{GeV} / c^{2}$. The R-parity violating couplings $\lambda_{122}$ and $\lambda_{121}$ are set to nonzero values to enable the decay of the $\tilde{\chi}^{0}$ into two charged leptons and a neutrino. The values of 
$\lambda_{122}$ and $\lambda_{121}$ are chosen to give a mean transverse decay length of approximately $20 \mathrm{~cm}$. The chosen masses explore the range to which CMS is currently sensitive.

Several simulated background samples are also generated with PYTHIA. The dominant background is DrellYan production of dileptons: prompt $e^{+} e^{-}$or $\mu^{+} \mu^{-}$pairs can be misidentified as displaced from the primary vertex due to detector resolution effects, and the production and decay of $\tau^{+} \tau^{-}$pairs can produce genuinely displaced leptons, although the probability that both $\tau$ leptons decay leptonically is small. Other simulated backgrounds are from $t \bar{t}, W / Z$ boson pair production (dibosons) with leptonic decays, and QCD multijet events. The last includes a potential background source from semileptonic decays of $b / c$-flavor hadrons. In all samples, the response of the detector is simulated using GEANT4 [21], and all the events are processed through the trigger emulation and event reconstruction chains of the CMS experiment.

\section{EVENT RECONSTRUCTION AND SELECTION}

To select $p p$ collisions, events are required to contain a primary vertex with at least four associated tracks and a position displaced from the nominal interaction point by no more than $2 \mathrm{~cm}$ in the direction transverse to the beam, and no more than $24 \mathrm{~cm}$ in the direction along the beam. Furthermore, to reject events produced by the interaction of beam-related protons with the LHC collimators, for events with at least ten tracks, the fraction of tracks classified as "high purity," as defined in Ref. [16], must exceed $25 \%$. When more than one primary vertex is reconstructed in an event, we select the one with the largest sum of the $p_{T}^{2}$ of the tracks associated to it.

In order to maximize the efficiency for reconstructing leptons from highly displaced vertices, we use lepton identification algorithms that are less stringent than the standard CMS algorithms, which are not needed to suppress the very low backgrounds in this analysis. Leptons are identified using tracks reconstructed in the tracker that are classified as high purity, and have pseudorapidity $|\eta|<2$. The latter requirement is imposed because the efficiency for finding tracks from displaced secondary vertices decreases at large $|\eta|$.

A track is identified as originating from an electron if its direction is consistent within a cone of size $\Delta R=$ $\sqrt{(\Delta \eta)^{2}+(\Delta \phi)^{2}}<0.1$ with an energy deposit in the ECAL that is reconstructed as a photon. Here, $\Delta \eta$ and $\Delta \phi$ are the differences between the track and the energy deposit in the ECAL in $\eta$ and $\phi$, respectively. The energy of the electron is taken from the energy deposit in the ECAL, since it is less affected by bremsstrahlung loss than the measurement of the track $p_{\mathrm{T}}$. Additional quality requirements are placed on the ECAL energy deposit to reject background from hadronic sources.
A track is identified as originating from a muon if it matches a muon candidate found within $\Delta R<0.1$. Here, $\Delta \eta$ and $\Delta \phi$ are the differences in direction between the track and the muon found by the trigger in $\eta$ and $\phi$, respectively.

The LL particle candidates are formed from pairs of charged-lepton candidates. In the muon channel, the two tracks must each have $p_{\mathrm{T}}>26 \mathrm{GeV} / c$ and be oppositely charged. In the electron channel, the higher (lower) $E_{\mathrm{T}}$ electron must satisfy $E_{\mathrm{T}}>40 \mathrm{GeV}(25 \mathrm{GeV})$. These thresholds are set slightly higher than the corresponding trigger requirements to ensure that the selected events have high trigger efficiency. In the dielectron channel, the two tracks must also satisfy $p_{\mathrm{T}}>36 \mathrm{GeV} / c(21 \mathrm{GeV} / c)$ if associated to the higher (lower) $E_{\mathrm{T}}$ electron. This $p_{\mathrm{T}}$ requirement, which is slightly lower than the corresponding $E_{\mathrm{T}}$ requirement placed on the ECAL energy deposit, suppresses electrons that emit large amounts of bremsstrahlung, and which thus tend to have poor impact parameter resolution. No charge requirement is applied to electrons, as the probability of mismeasuring the charge is non-negligible for high- $p_{\mathrm{T}}$ electrons.

To reject promptly produced particles, the tracks are required to have a transverse impact parameter significance with respect to the primary vertex of $\left|d_{0}\right| / \sigma_{d}>12$, where $\sigma_{d}$ is the uncertainty on $\left|d_{0}\right|$. This value is chosen to give an expected background significantly below one event, which gives the best signal sensitivity for the vast majority of the LL particle lifetimes considered in this paper. Both lepton candidates are required to be isolated, to reject background from jets. Specifically, a hollow isolation cone is constructed around each candidate, with a radius $0.04<\Delta R<$ 0.3 for electrons and $0.03<\Delta R<0.3$ for muons. Within this isolation cone, the ratio of the scalar $\sum p_{\mathrm{T}}$ of all tracks with $p_{\mathrm{T}}>1 \mathrm{GeV} / c$, excluding the other lepton candidate, to the $p_{\mathrm{T}}$ of the lepton, must be less than 0.1 .

The two tracks are fitted to a common vertex, which is required to have $\chi^{2} /$ dof $<10(5)$ in the electron (muon) channel. To ensure that the candidate tracks were produced at this vertex, we require that the number of hits, between the center of CMS and the vertex position, that is assigned to the tracks is no more than 1 , and that the number of missing hits on the tracks between the vertex position and the outer envelope of the tracker is no more than 3 (4) in the electron (muon) channel, where in both cases the numbers are summed over both tracks. A missing hit is defined as occurring when a track passes through an active sensor without being assigned a reconstructed hit. To eliminate background from $J / \psi$ and $\Upsilon$ decays, and from $\gamma$ conversions, LL particle candidates are required to have a dilepton invariant mass larger than $15 \mathrm{GeV} / c^{2}$.

Cosmic ray muons may be reconstructed as back-to-back tracks. To reject them, the three-dimensional opening angle between the two muons must be less than 2.48 radians. This requirement is slightly tighter than the requirement in the 
trigger. Background from misidentified leptons is reduced by requiring that the two lepton candidates are not both matched to the same trigger object or offline photon. Owing to the difficulty of modeling the low trigger efficiency for closely spaced muon pairs, the two muons are required to be separated by $\Delta R>0.2$.

Finally, the signed difference in azimuthal angles, $\Delta \Phi$, between the dilepton momentum vector, $\bar{p}_{\ell \ell}$, and the vector from the primary vertex to the dilepton vertex, $\bar{v}_{\ell \ell}$, is required to satisfy $|\Delta \Phi|<\pi / 2$, where $\Delta \Phi$ is measured in the range $0<\Delta \Phi<\pi$. Dilepton candidates satisfying all other selection requirements, but with $|\Delta \Phi|>\pi / 2$, are used to define a control region, as detailed in Sec. V.

Events containing at least one LL particle candidate that passes all selection requirements are accepted. Where more than one candidate is found in an event, the one with largest $\left|d_{0}\right| / \sigma_{d}$ is chosen. The $\left|d_{0}\right| / \sigma_{d}$ of a candidate is defined as the minimum of the two $\left|d_{0}\right| / \sigma_{d}$ values of the leptons that comprise it.

The overall signal efficiency is defined as the fraction of events in which at least one dilepton candidate passes all selection criteria. It is determined from the simulated signal samples, separately for the electron and muon channels, and independently for two different classes of events: first for events in which only one LL particle ( $X$ or $\left.\tilde{\chi}^{0}\right)$ decays to the chosen lepton species, defining efficiency $\epsilon_{1}$, and second for events in which both LL particles decay to the chosen lepton species, defining efficiency $\epsilon_{2}$. The efficiencies are estimated for LL particle lifetimes corresponding to mean transverse decay lengths in the range of $200 \mu \mathrm{m}-200 \mathrm{~m}$, by reweighting the simulated signal events. The maximum value of $\epsilon_{1}$, which is attained for $H \rightarrow X X$ with $m_{H}=1000 \mathrm{GeV} / c^{2}, m_{X}=150 \mathrm{GeV} / c^{2}$, and $c \tau=1 \mathrm{~cm}$, is approximately $36 \%(46 \%)$ in the electron (muon) channel, but it becomes significantly smaller at lower $H$ masses or at longer and shorter lifetimes. For example, if $c \tau$ is increased to $20 \mathrm{~cm}$ for this set of masses, then $\epsilon_{1}$ drops to $14 \%$ (20\%) in the electron (muon) channel. The efficiencies in the muon channel are generally higher because of the lower $p_{\mathrm{T}}$ thresholds compared to the corresponding thresholds in the electron channel.

In order to reduce the model dependence of our results, it is useful to define a set of acceptance criteria that specifies the LL particles decaying to dilepton final states that can be reconstructed in the CMS detector. Specifically, the generated transverse decay length of the LL particle should be no more than $50 \mathrm{~cm}$, and the generated electrons (muons) should satisfy the same $E_{\mathrm{T}}\left(p_{\mathrm{T}}\right)$ and $\eta$ requirements that are applied to the reconstructed electrons (muons), which are listed earlier in this section. The acceptance $A$ is defined as the fraction of LL particle decays that pass the acceptance criteria. Reevaluating the signal efficiency $\epsilon_{1}$, using only LL particle decays within the acceptance, yields $\epsilon_{1} / A$, which is larger than $\epsilon_{1}$. For example, for $m_{H}=1000 \mathrm{GeV} / c^{2}, m_{X}=150 \mathrm{GeV} / c^{2}$, and $c \tau=1 \mathrm{~cm}$, the value of $\epsilon_{1} / A$ is approximately $44 \%(58 \%)$ in the electron (muon) channel. More importantly, the efficiency defined in this way shows much less dependence on the choice of signal model; e.g. for this same choice of masses, but with $c \tau=20 \mathrm{~cm}$, it falls only to $28 \%$ (40\%) in the electron (muon) channel.

\section{BACKGROUND ESTIMATION AND ASSOCIATED SYSTEMATIC UNCERTAINTIES}

To estimate the background, we consider the quantities $\bar{v}_{\ell \ell}, \bar{p}_{\ell \ell}$ and $\Delta \Phi$ defined in Sec. IV. For signal events, $\bar{v}_{\ell \ell}$ corresponds to the flight direction of the LL particle, and assuming that the dilepton system produced when the LL particle decays is usually boosted with respect to its flight direction, the direction of $\bar{p}_{\ell \ell}$ is correlated with that of $\bar{v}_{\ell \ell}$. In contrast, for background events, $\bar{v}_{\ell \ell}$ does not correspond to the flight direction of any long-lived particle, so its angular distribution with respect to $\bar{p}_{\ell \ell}$ should not show any forward-backward asymmetry. For example, in the case of Drell-Yan production of $\ell^{+} \ell^{-}, \bar{v}_{\ell \ell}$ is determined only by effects such as detector resolution or primary vertex misassignment. Although in the case of Drell-Yan production of $\tau^{+} \tau^{-}$, leptonic products of the $\tau$-lepton decays may have significant values of $\left|d_{0}\right| / \sigma_{d}$ because of the nonzero lifetime of the $\tau$ lepton, a vertex reconstructed from two such leptons would not correspond to a genuine particle decay vertex. Processes such as nonprompt $J / \psi$ decay or $\gamma$ conversions, which can give rise to genuine displaced dilepton vertices, are eliminated by the requirement on the minimum dilepton mass. Cosmic ray background is reduced to negligible levels via the dimuon opening angle requirement that rejects back-to-back muons.

Therefore if we define a signal region with $|\Delta \Phi|<\pi / 2$ and a control region with $|\Delta \Phi|>\pi / 2$, we expect that signal events will populate the former region, while background events will be equally distributed between the two. Consequently, we can use the distribution of events in the control region to derive a data-driven estimate of the background expected in the signal region.

Figure 1 shows the $\left|d_{0}\right| / \sigma_{d}$ distribution of the simulated events in the signal and control regions. Each of the simulated backgrounds is statistically consistent with being symmetrically divided between the two regions. The expected background is predominantly Drell-Yan dilepton production, with some contribution from QCD multijets. Any discrepancies between data and simulation are unimportant since the analysis uses a data-driven background estimate. They may arise because of imperfect modeling in the simulation or because of the large statistical uncertainty in the simulated QCD multijet background. The multijet background near $\left|d_{0}\right| / \sigma_{d}=6$ in the top, right-hand plot corresponds to a single simulated event. We observe that more than $97 \%$ (95\%) of simulated signal events fall into the signal region for the $X \rightarrow \ell^{+} \ell^{-}\left(\tilde{\chi}^{0} \rightarrow \ell^{+} \ell^{-} \nu\right)$ model for all the samples considered. 

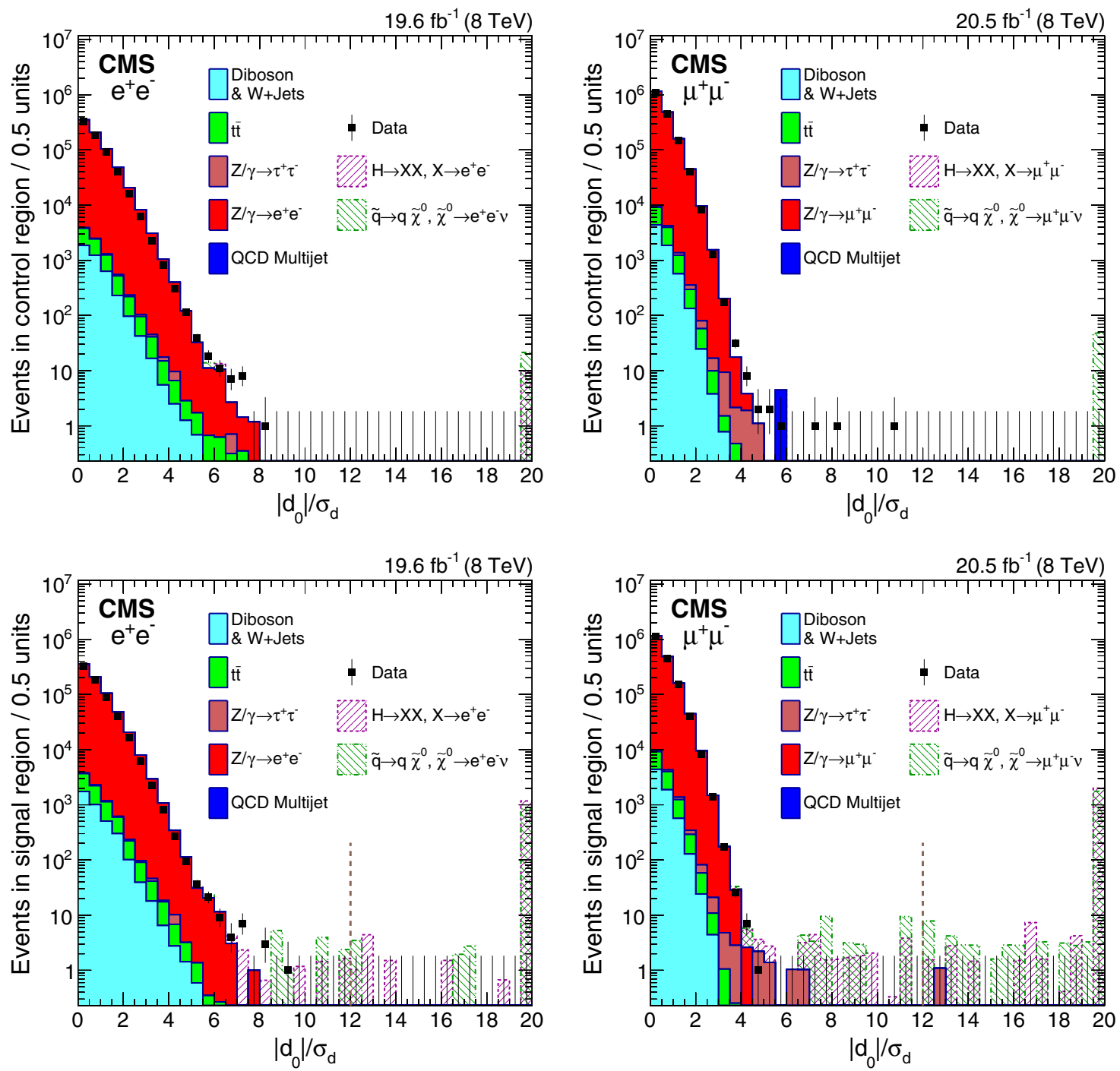

FIG. 1 (color online). The $\left|d_{0}\right| / \sigma_{d}$ distribution for the electron (left) and muon (right) channels, shown in the top row for events in the control region $(|\Delta \Phi|>\pi / 2)$ and in the bottom row for events in the signal region $(|\Delta \Phi|<\pi / 2)$. Of the two leptons forming a candidate, the distribution of the one with the smallest $\left|d_{0}\right| / \sigma_{d}$ is plotted. The solid points indicate the data, the shaded histograms are the simulated background, and the hashed histograms show the simulated signal. The histogram corresponding to the $H \rightarrow X X$ model is shown for $m_{H}=1000 \mathrm{GeV} / c^{2}$ and $m_{X}=350 \mathrm{GeV} / c^{2}$. The histogram corresponding to the $\tilde{\chi}^{0} \rightarrow \ell^{+} \ell^{-} \nu$ model is shown for $m_{\tilde{q}}=350 \mathrm{GeV} / c^{2}$ and $m_{\tilde{\chi}^{0}}=140 \mathrm{GeV} / c^{2}$. The background histograms are stacked, and each simulated signal sample is independently stacked on top of the total simulated background. The $d_{0}$ corrections for residual tracker misalignment, discussed in the text, have been applied. The vertical dashed line shows the selection requirement $\left|d_{0}\right| / \sigma_{d}>12$. Any entries beyond the right-hand side of a histogram are shown in the last visible bin of the histogram.

Besides using simulated events, we validate this method by comparing the $\left|d_{0}\right| / \sigma_{d}$ distribution in the signal region with the one in the control region using data at $\left|d_{0}\right| / \sigma_{d}$ values for which the sample is background dominated. Figure 2 shows the tail-cumulative distributions, which are defined as integrals from the plotted value to infinity, of $\left|d_{0}\right| / \sigma_{d}$ in the signal and control regions. However, the region with $\left|d_{0}\right| / \sigma_{d}>6$ (4.5) in the electron (muon) channel is excluded from the integral, to ensure that the signal region is background dominated. No statistically significant difference between the two regions is seen.
We observe zero events in data with $\left|d_{0}\right| / \sigma_{d}>12$ in the control region, and this determines the probability distribution of the expected background level, as discussed in Sec. VII. The systematic uncertainty in this estimate is defined below.

Residual misalignment of the tracker is the only effect that can cause the expected background to differ significantly in the signal and control regions. This effect is largely removed by applying corrections, described below, to the conventionally signed [16] transverse and longitudinal $\left(z_{0}\right)$ impact parameters of all tracks. The mean offset 

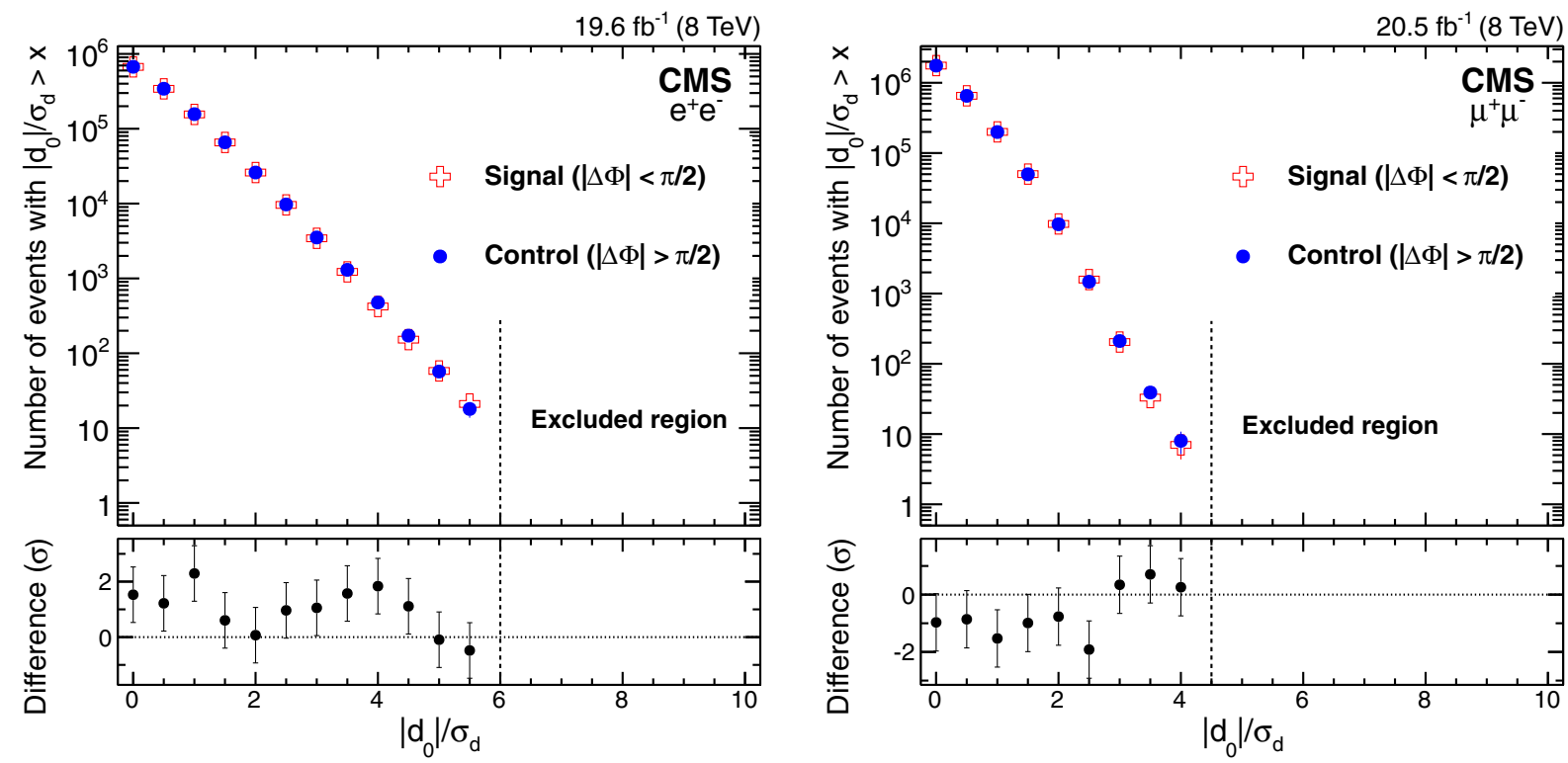

FIG. 2 (color online). Comparison of the tail-cumulative distributions of $\left|d_{0}\right| / \sigma_{d}$ for data in the signal region $(|\Delta \Phi|<\pi / 2)$ and the control region $(|\Delta \Phi|>\pi / 2)$ for the electron channel (left) and the muon channel (right). The $d_{0}$ corrections for residual tracker misalignment, discussed in the text, have been applied. Of the two leptons forming a candidate, the distribution of the one with the smallest $\left|d_{0}\right| / \sigma_{d}$ is plotted. The bottom panels show the statistical significance of the difference between the distributions in the signal and control regions.

from zero of the signed $d_{0}$ and $z_{0}$ of prompt muon tracks (i.e. $\left|d_{0}\right|$ and $\left|z_{0}\right|$ below $\left.500 \mu \mathrm{m}\right)$ is measured as a function of the track $\eta$ and $\phi$, and also as a function of run period. This bias, which arises from residual misalignment and is always less than $5 \mu \mathrm{m}$, is then subtracted from the measured impact parameters of individual tracks. To verify that this method is reliable, we first apply it to a data sample reconstructed with a preliminary alignment calibration, much inferior to the final alignment calibration used for the latest CMS data sets. In this sample, we observe a significant asymmetry between the control and signal regions, most of which disappears when the impact parameter corrections are applied.

Two approaches, described below, are used to assess the effect of any remaining systematic uncertainty in the background estimate due to misalignment. The first makes a direct measurement of the background asymmetry in the $\left|d_{0}\right| / \sigma_{d}$ distribution. The second checks how much, if at all, the LL particle search results change if the impact parameter corrections are removed.

The first approach measures the systematic uncertainty remaining after the impact parameter corrections have been applied, by comparing the $\left|d_{0}\right| / \sigma_{d}$ distributions in the two regions with $\Delta \Phi<0$ and $\Delta \Phi>0$. Both signal and background are expected to be equally divided between these two regions, so any significant asymmetry between them can only arise through systematic effects. We measure the size of this asymmetry by comparing the ratio of the number of events in the tail-cumulative distribution of $\left|d_{0}\right| / \sigma_{d}$ in the region $\Delta \Phi<0$ with that in the region $\Delta \Phi>0$. Points at $\left|d_{0}\right| / \sigma_{d}$ values with very few events, such that the relative statistical uncertainty in this ratio is greater than $30 \%$, are excluded since they would not provide a precise estimate of the systematic uncertainty. The maximum difference of the ratio from unity for all remaining points is then taken to be the systematic uncertainty. Using this procedure, we obtain a systematic uncertainty of 11 and $21 \%$ in the electron and muon channels, respectively, in the estimated amount of background.

The second approach addresses a potential issue with the first method, namely that it measures the systematic uncertainty in the background normalization at lower values of $\left|d_{0}\right| / \sigma_{d}$ than are used in our standard selection. In the data, the bias on the track $d_{0}$ due to misalignment is less than $5 \mu \mathrm{m}$, whereas our $\left|d_{0}\right| / \sigma_{d}>12$ requirement typically corresponds to a selection on $\left|d_{0}\right|$ of approximately $180 \mu \mathrm{m}$. This suggests that misalignment should not be a significant effect at large $\left|d_{0}\right| / \sigma_{d}$. Nonetheless, to allow for the possibility that it might be, we employ the second approach; namely, when computing our final limits, we do so twice, once with the impact parameter corrections applied, and once without them, and then take the worse limits as our final result. This should be conservative, given that as stated above, the impact parameter corrections remove the majority of any asymmetry caused by misalignment. In practice, the misalignment is so small that these two sets of limits are identical.

\section{SYSTEMATIC UNCERTAINTIES AFFECTING THE SIGNAL}

The systematic effects influencing the signal efficiency arise from uncertainties in the efficiency of reconstructing 
TABLE I. Systematic uncertainties affecting the signal efficiency over the two signal models and all mass values considered. In all cases, the uncertainty specified is a relative uncertainty. The next-to-leading-order (NLO) uncertainty is significant only for the $H \rightarrow X X$ model with $m_{H}=125 \mathrm{GeV} / c^{2}$. The relative uncertainty in the integrated luminosity is $2.6 \%$.

\begin{tabular}{lc}
\hline \hline Source & Uncertainty \\
\hline Pileup modeling & $2 \%$ \\
Parton distribution functions & $<1 \%$ \\
Renormalization and factorization scales & $<0.5 \%$ \\
Track reconstruction efficiency from & $6.1 \%$ \\
$\quad$ cosmic ray muons & \\
Track reconstruction efficiency in high & $3.5 \%$ \\
$\quad$ hit occupancy environment & \\
Track reconstruction efficiency loss & $5.8 \%$ \\
$\quad$ due to bremsstrahlung $(e$ only) & $1.7 \%(e), 6.2 \%(\mu)$ \\
Trigger efficiency & $5-7 \%$ \\
NLO effects (only for the & \\
$m_{H}=125 \mathrm{GeV} / c^{2}$ case) & \\
\hline \hline
\end{tabular}

tracks from displaced vertices, the trigger efficiency, the modeling of pileup (i.e. additional $p p$ collisions in the same bunch crossing), the parton distribution function (PDF) sets, the renormalization and factorization scales used in generating simulated events, and the effect of higher-order QCD corrections.

Table I summarizes the non-negligible sources of systematic uncertainty affecting the signal efficiency. These

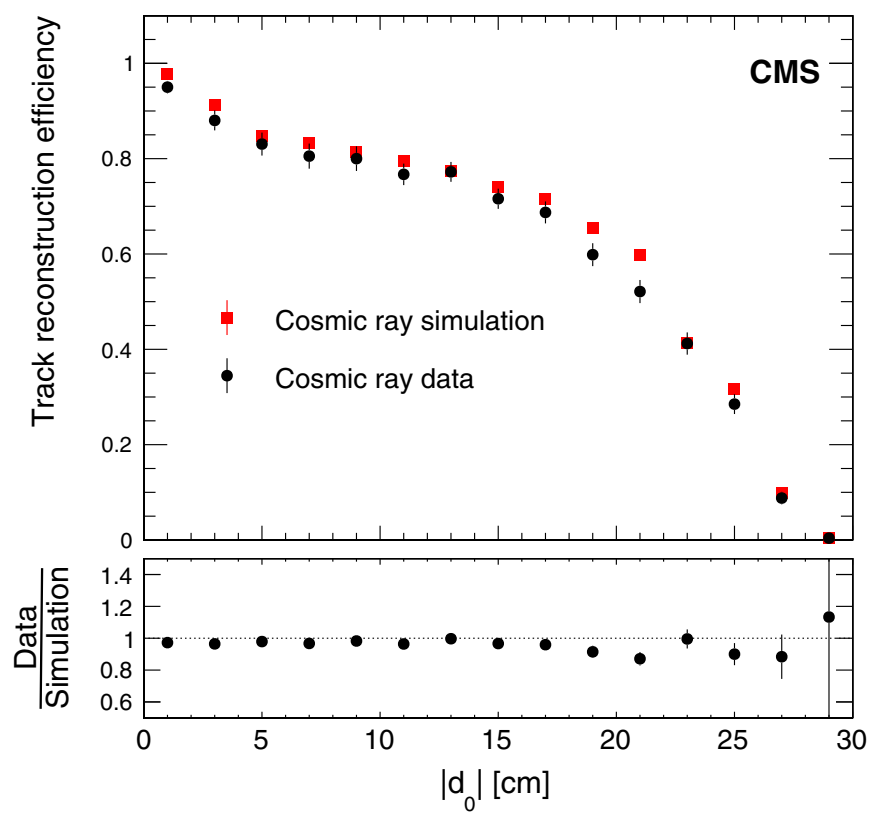

are discussed in more detail below. The most important sources are those related to the track reconstruction efficiency. The relative uncertainty in the measurement of the integrated luminosity is $2.6 \%$ [22].

Varying the modeling of the pileup within its estimated uncertainties yields a relative change in the signal selection efficiency of less than $2 \%$, irrespective of the mass point chosen. The relative uncertainty due to the choice of PDF set is studied using the PDF4LHC prescription [23] and is less than $1 \%$ for all mass points. The dependence of the acceptance on the choice of the renormalization and factorization scales, which are chosen to be equal, is found to be well below $0.5 \%$ when they are varied by a factor of 0.5 or 2 . These uncertainties are applied in the cross section limit calculation.

\section{A. Track finding efficiency}

Three methods are used to assess if the efficiency to reconstruct displaced tracks is correctly modeled by the simulation. The first method consists of a direct measurement of the efficiency to reconstruct isolated, displaced tracks, using cosmic ray muons. Events are selected from dedicated running periods with no beam present, and the cosmic ray muons are reconstructed by combining the hits in the muon detectors from opposite halves of the CMS detector. The efficiency to reconstruct, in the tracker, a track associated with a cosmic ray muon, as a function of the transverse and longitudinal impact parameters, is shown in Fig. 3. The systematic uncertainty on the dilepton

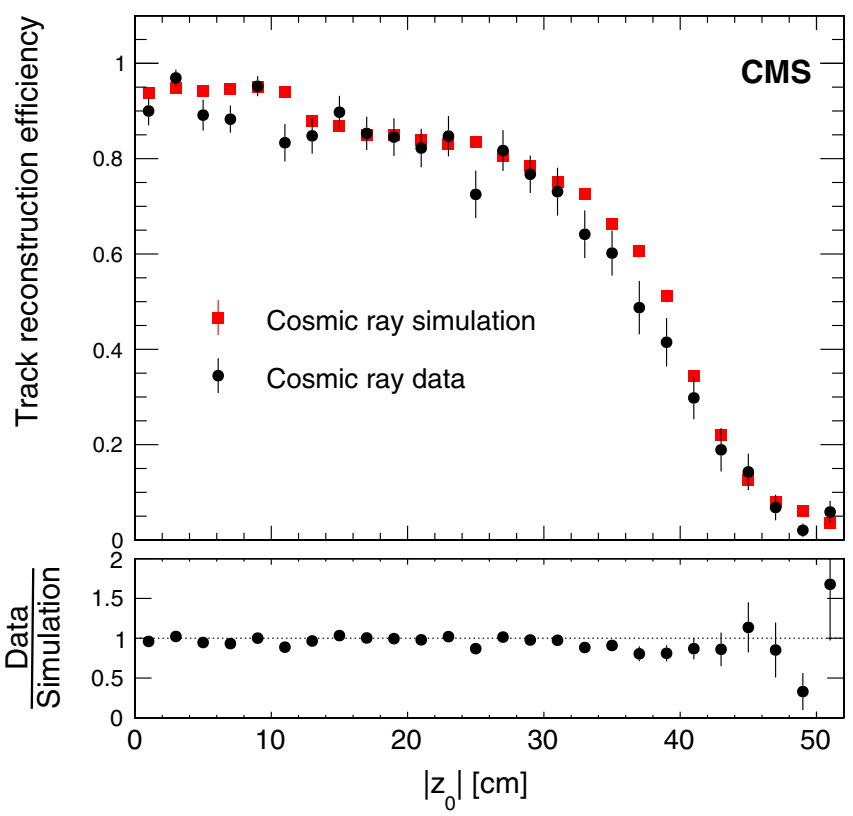

FIG. 3 (color online). Efficiency to find a track in the tracker, measured using cosmic ray muons reconstructed in the muon detectors, as a function of the transverse (left) and longitudinal (right) impact parameters (relative to the nominal interaction point of CMS). The efficiency is plotted in bins of $2 \mathrm{~cm}$ width. For the left plot, the longitudinal impact parameter $\left|z_{0}\right|$ is required to be less than $10 \mathrm{~cm}$, and for the right plot, the transverse impact parameter $\left|d_{0}\right|$ must be less than $4 \mathrm{~cm}$. The bottom panels show the ratio of the efficiency in data to that in simulation. The uncertainties in the simulation are smaller than the size of the markers and are not visible. 
efficiency is estimated as follows. We use the measured track reconstruction efficiency to estimate the efficiency to reconstruct a pair of leptons of given impact parameters. We then weight this efficiency according to the impact parameter distributions of the dileptons in the simulated signal Monte Carlo samples. The ratio of the estimated efficiency per dilepton candidate in data to simulation differs from unity by no more than $6.1 \%$ for any of the samples considered, so this value is taken as the systematic uncertainty.

A second method is used to study how the presence of a high density of tracker hits around displaced leptons degrades the track reconstruction performance. This method takes cosmic ray muon data, where each muon is reconstructed in the muon detectors and is successfully associated to a track reconstructed in the tracker. It embeds each of these tracks and its associated hits into a high-occupancy $p p$ collision data event, and measures the fraction of these embedded tracks that can still be successfully reconstructed in this environment as a function of their impact parameters. The results are compared with those obtained by embedding tracks from simulated cosmic events in simulated $p p$ collisions. The same procedure described at the end of the preceding paragraph is applied, and leads us to conclude that the efficiency per candidate has an additional systematic uncertainty, related to the track reconstruction efficiency in a high hit density environment, of 3.5\%.

A third method [9] uses charged pions from $K_{S}^{0}$ decay to establish that the track reconstruction efficiency is simulated with a relative systematic uncertainty of 5\%. Since this method is mainly sensitive to the track reconstruction efficiency of low- $p_{\mathrm{T}}$ hadrons in jets, it is used only to provide additional reassurance that the displaced track reconstruction efficiency is well modeled.

These methods do not explicitly measure the track reconstruction efficiency for electrons, where an additional systematic uncertainty must be considered. For the leptons from LL particle decay in the simulated signal samples, the track reconstruction efficiency for the electrons is about $78 \%$ that of the muons, where the difference arises from the emission of bremsstrahlung. This difference does not show significant variation with respect to the transverse decay length of the LL particle. The material budget of the tracker is modeled in simulation to an accuracy of $<10 \%$ [24]. Since the amount of bremsstrahlung should be proportional to the amount of material in the tracker, this implies a corresponding relative uncertainty in the difference between the track reconstruction efficiencies for electrons and muons. This leads to a bremsstrahlung-related relative uncertainty in the tracking efficiency for electrons of $0.22 \times 10 \% /(1-0.22)=2.9 \%$, where the denominator arises because this uncertainty is measured relative to the tracking efficiency for electrons, not muons. The corresponding systematic uncertainty for the dielectron candidates, which have two tracks, is twice as large, namely 5.8\%.

\section{B. Trigger efficiency}

The trigger efficiency is measured using the "tag-andprobe" method [25]. In the muon channel, $Z$ boson decays to dimuons are reconstructed in data collected with single-muon triggers. They are then used to measure the efficiency for a muon to pass the selection criteria of one leg of the dimuon trigger used in this analysis. The dimuon trigger efficiency is then obtained as the square of this single-muon efficiency, which assumes that there is no correlation in efficiency between the two leptons. This is generally a good assumption except for dimuons separated by $\Delta R<0.2$, which are excluded because the trigger is inefficient for closely spaced dimuons. In the electron channel, the method is similar, but since the two legs of the trigger for this channel have different $E_{\mathrm{T}}$ thresholds, the efficiency of each leg is measured separately. In data, the trigger efficiency is essentially $100 \%$ for electrons satisfying the analysis selection. Under the same conditions, the efficiency for muons with a $p_{\mathrm{T}}$ of about $26 \mathrm{GeV} / c$ is above $70 \%$ and it reaches a plateau of approximately $85 \%$ for $p_{\mathrm{T}}>40 \mathrm{GeV} / c$.

The systematic uncertainty associated with the trigger efficiency is evaluated by taking the difference between the efficiency estimates from data and simulation, which yields a total relative uncertainty of $1.7 \%$ for the electron channel and $6.2 \%$ for the muon channel. To ensure that the trigger efficiencies obtained from the sample of $Z$ bosons, in which the leptons are prompt, are also valid for leptons from LL particle decay, we examine the trigger efficiency in simulated signal events as a function of the lifetime of the LL particles. For LL particles passing the acceptance criteria defined in Sec. IV, no statistically significant dependence of the trigger efficiency on their lifetime is seen. Therefore, systematic uncertainties related to this source may be neglected in comparison to the systematic uncertainties on the trigger efficiency quoted above.

\section{Effect of higher-order QCD corrections}

For the $H \rightarrow X X$ sample with $m_{H}=125 \mathrm{GeV} / c^{2}$, the leptons from the $X$ boson decay have a combined efficiency of only a few percent for passing the lepton $p_{\mathrm{T}}$ requirements. For this reason the signal efficiency at this mass is sensitive to the modeling of the Higgs boson $p_{\mathrm{T}}$ spectrum, which may in turn be influenced by higher-order QCD corrections. To evaluate this effect, we reweight the leading-order Higgs boson $p_{\mathrm{T}}$ spectrum from our signal sample to match the corresponding Higgs boson $p_{\mathrm{T}}$ spectrum evaluated at NLO [26-28]. For $m_{H}=125 \mathrm{GeV} / c^{2}$ and $m_{X}=$ $20(50) \mathrm{GeV} / c^{2}$ the signal efficiency changes by $5 \%$ (7\%). This change is taken as an additional systematic uncertainty in the efficiency for the case $m_{H}=$ $125 \mathrm{GeV} / c^{2}$. For the larger $H$ masses that we consider, and also for the neutralino channel, where a similar study was performed, the corresponding systematic uncertainty is below $0.5 \%$, and hence neglected. 

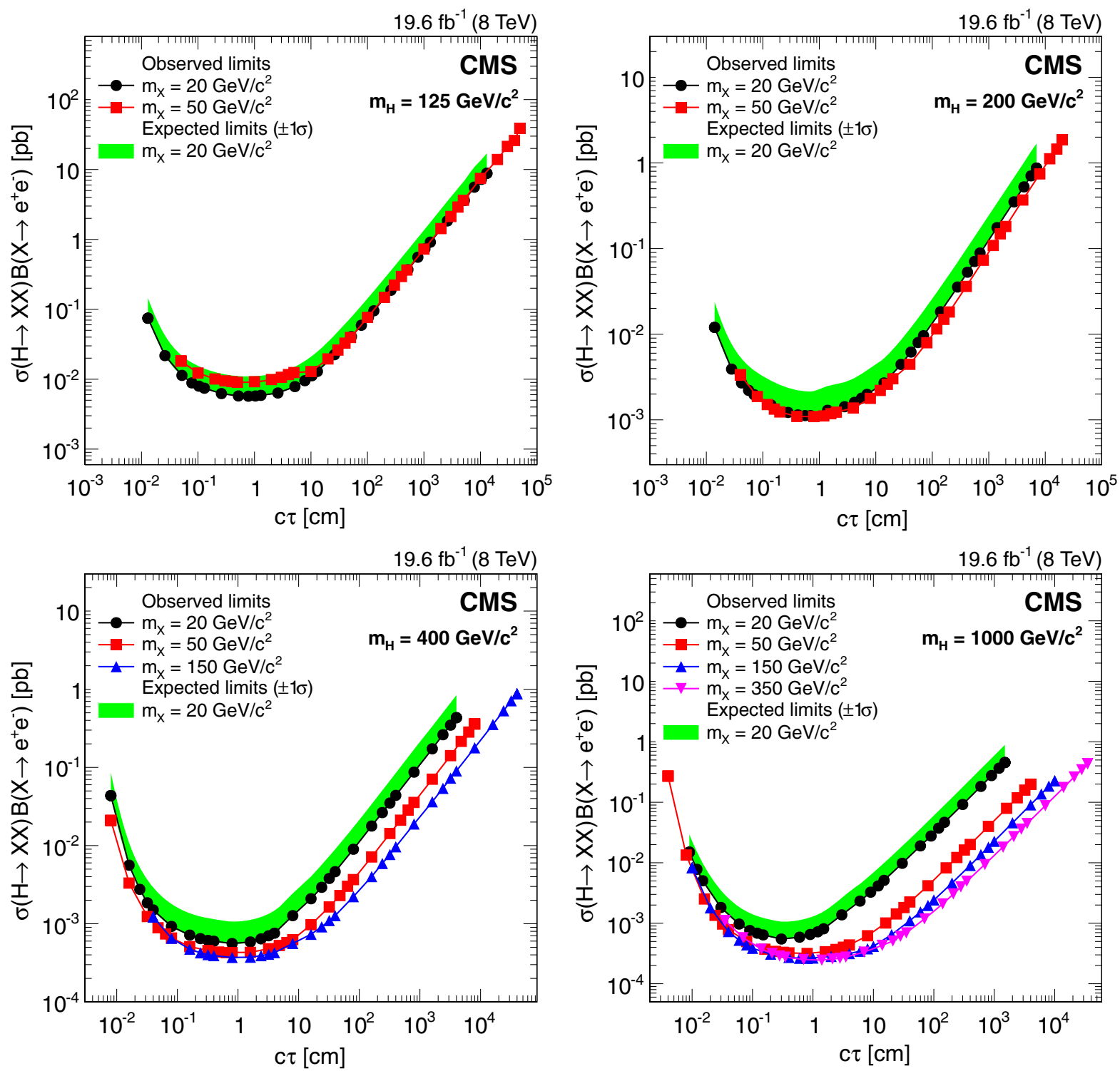

FIG. 4 (color online). The 95\% C.L. upper limits on $\sigma(H \rightarrow X X) \mathcal{B}\left(X \rightarrow e^{+} e^{-}\right)$, as a function of the mean proper decay length of the $X$ boson, for Higgs boson masses of 125 (top left), 200 (top right), 400 (bottom left), and $1000 \mathrm{GeV} / c^{2}$ (bottom right). In each plot, results are shown for several $X$ boson mass hypotheses. The shaded band shows the $\pm 1 \sigma$ range of variation of the expected 95\% C.L. limits for the case of a $20 \mathrm{GeV} / c^{2} X$ boson mass. Corresponding bands for the other $X$ boson masses, omitted for clarity of presentation, show similar agreement with the respective observed limits.

\section{RESULTS}

Events from background sources are equally likely to populate the signal and control regions, whereas any events arising from LL particles will populate almost exclusively the signal region. In consequence, the presence of a signal in the data would reveal itself as a statistically significant excess of events in the signal region compared to the control region. After all selection requirements are applied, no events are found in the signal or control regions in either the electron or muon channel. There is thus no statistically significant excess. The $\left|d_{0}\right| / \sigma_{d}$ distributions of events in the signal and control regions were shown in Fig. 1.
We set 95\% confidence level (C.L.) upper limits on the signal processes using the Bayesian method described in Ref. [29]. The limits are determined from a comparison of the number of events observed in the signal region with the number expected in the signal plus background hypothesis.

The limit calculation takes into account the systematic uncertainties in the signal yield, described in Sec. VI, by introducing nuisance parameters for each of the uncertainties that are marginalized through an integration over their log-normal prior distributions. The expected number of background events $\mu_{B}$ in the control region, and hence also in the signal region, is an additional nuisance parameter. It 

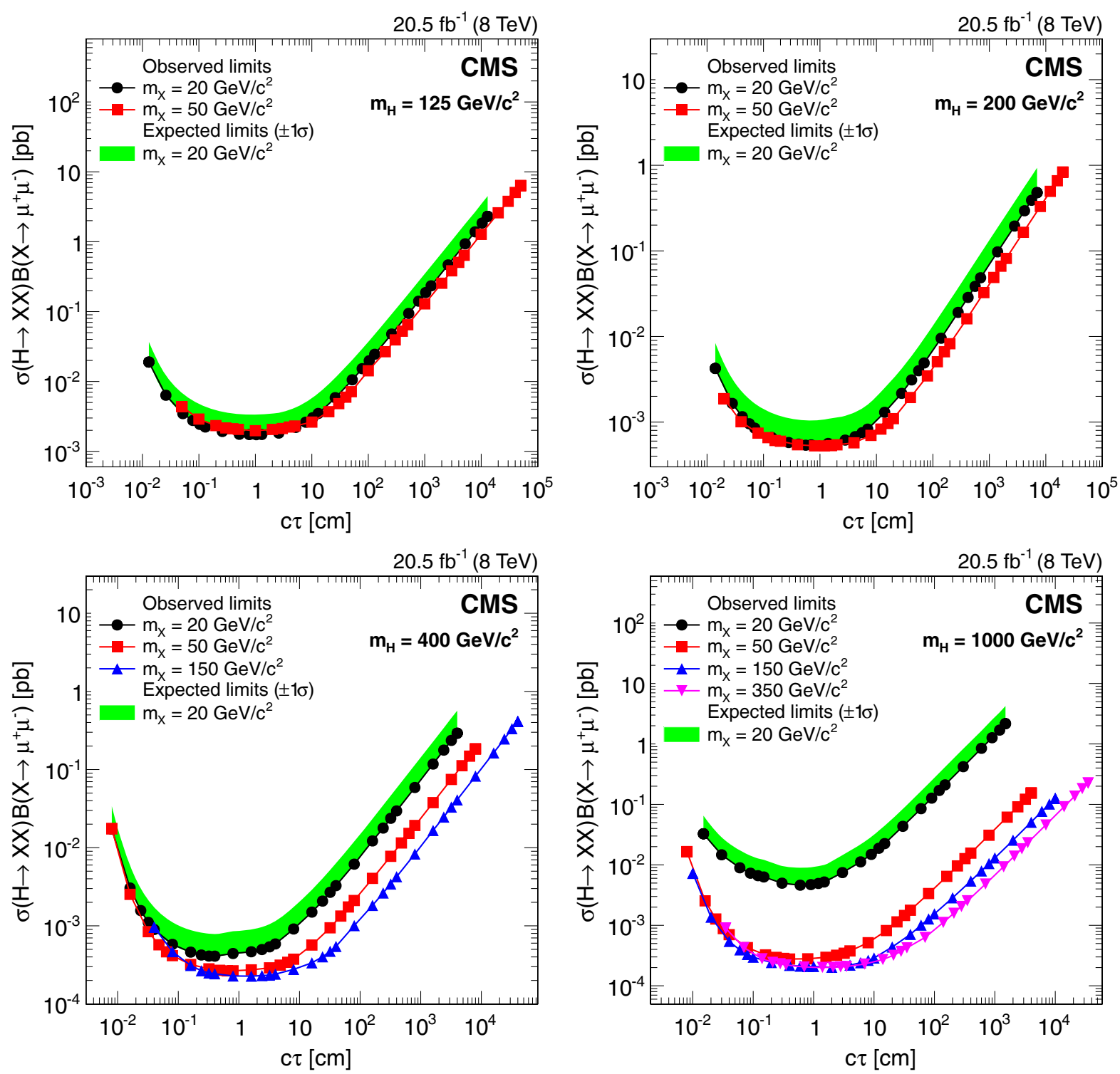

FIG. 5 (color online). The 95\% C.L. upper limits on $\sigma(H \rightarrow X X) \mathcal{B}\left(X \rightarrow \mu^{+} \mu^{-}\right)$, as a function of the mean proper decay length of the $X$ boson, for Higgs boson masses of 125 (top left), 200 (top right), 400 (bottom left), and $1000 \mathrm{GeV} / c^{2}$ (bottom right). In each plot, results are shown for several $X$ boson mass hypotheses. The shaded band shows the $\pm 1 \sigma$ range of variation of the expected 95\% C.L. limits for the case of a $20 \mathrm{GeV} / c^{2} X$ boson mass. Corresponding bands for the other $X$ boson masses, omitted for clarity of presentation, show similar agreement with the respective observed limits.

is constrained by the observed number of events $N_{C}$ in the control region. Its probability distribution $p\left(\mu_{B} \mid N_{C}\right)$ is given by

$$
p\left(\mu_{B} \mid N_{C}\right)=\frac{\mu_{B}^{N_{C}}}{N_{C} !} \exp \left(-\mu_{B}\right)
$$

as can be shown using Bayesian methodology assuming a flat prior in $\mu_{B}$ [29]. The expected background in the signal region may differ from that in the control region, as a result of tracker misalignment. This is taken into account as described in Sec. V, by including an appropriate systematic uncertainty, and by evaluating the limits twice, once with and once without correcting the track impact parameters for tracker misalignment, and taking the worse of these two sets of limits as the result.

If a genuine signal were present, it would give rise to an excess of events in the signal region with an expected number of

$$
\mu_{S}=\mathcal{L} \sigma\left[2 \mathcal{B}(1-\mathcal{B}) \epsilon_{1}+\epsilon_{2} \mathcal{B}^{2}\right](1-f),
$$

where $\mathcal{L}$ is the integrated luminosity, $\epsilon_{(1,2)}$ are the signal efficiencies defined in Sec. IV, $\sigma$ is the production cross section of $H \rightarrow X X$ (or $\left.\tilde{q} \tilde{q}^{*}+\tilde{q} \tilde{q}\right)$ and $\mathcal{B}$ is the branching 

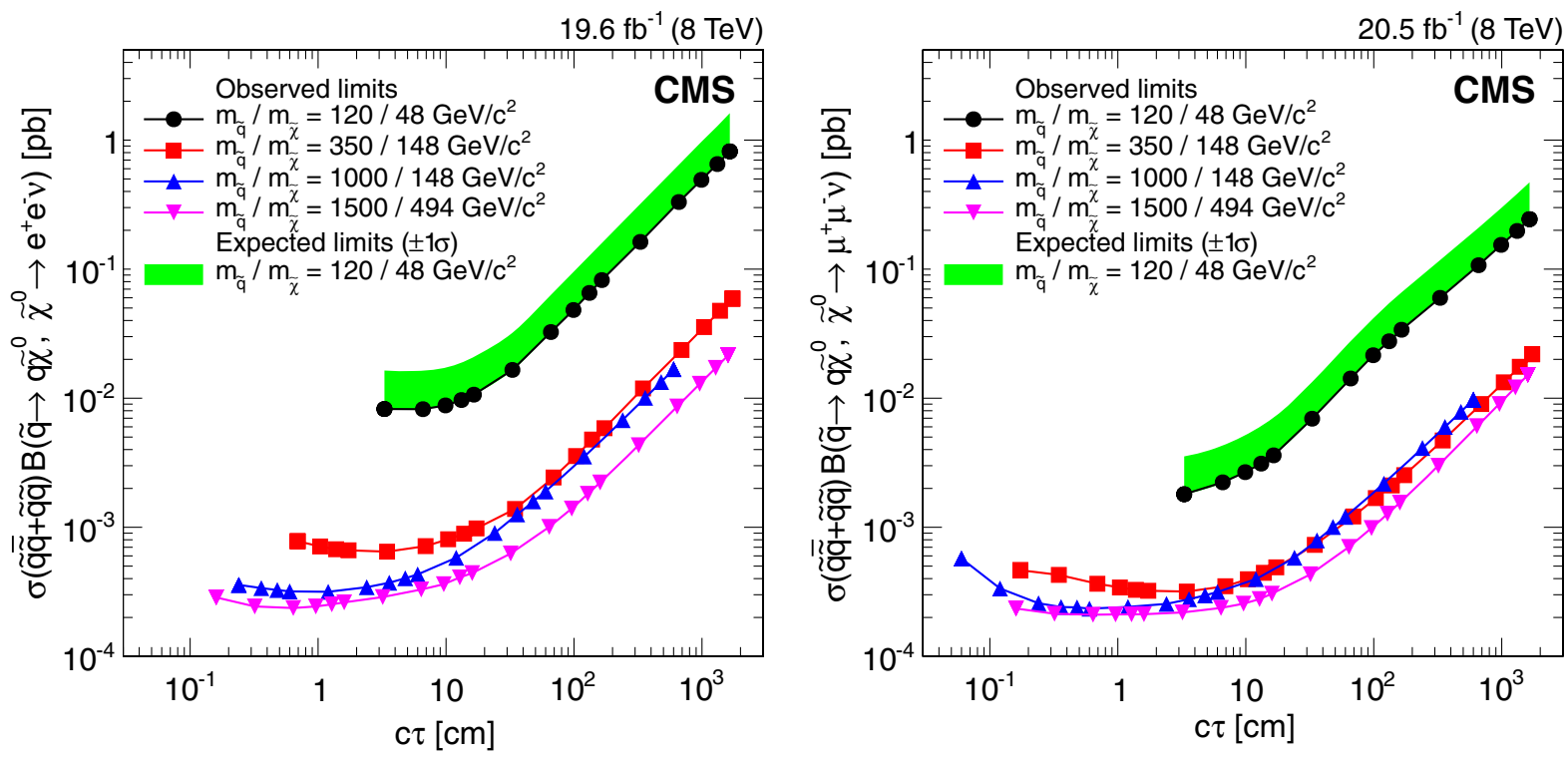

FIG. 6 (color online). The 95\% C.L. upper limits on $\sigma\left(\tilde{q} \tilde{q}^{*}+\tilde{q} \tilde{q}\right) \mathcal{B}\left(\tilde{q} \rightarrow q \tilde{\chi}^{0}, \tilde{\chi}^{0} \rightarrow \ell^{+} \ell^{-} \nu\right)$ for the electron (left), and muon (right) channels, as a function of the mean proper decay length of the neutralino. The shaded band shows the $\pm 1 \sigma$ range of variation of the expected $95 \%$ C.L. limits for the case of a $120 \mathrm{GeV} / c^{2}$ squark and a $48 \mathrm{GeV} / c^{2}$ neutralino mass. Corresponding bands for the other squark and neutralino masses, omitted for clarity of presentation, show similar agreement with the respective observed limits.

fraction for the decay $X \rightarrow \ell^{+} \ell^{-}$(or $\tilde{q} \rightarrow q \tilde{\chi}^{0}$, $\left.\tilde{\chi}^{0} \rightarrow \ell^{+} \ell^{-} \nu\right)$. The parameter $f$ is the mean number of signal events expected to fall in the control region for each signal event in the signal region. This fraction is very small, being less than $3 \%$ for all the $X \rightarrow \ell^{+} \ell^{-}$samples and less than $5 \%$ for all the $\tilde{\chi}^{0} \rightarrow \ell^{+} \ell^{-} \nu$ samples considered here. Its effect is to reduce slightly the effective signal efficiency, by causing some of the signal to be misinterpreted as background. One expects $\epsilon_{2} \geq 1-\left(1-\epsilon_{1}\right)^{2}$, where the two terms are equal if the efficiency to select each of the two LL particles in an event is independent of the other, or the first term is larger if the presence of one LL particle increases the efficiency to select the other (as can happen if one lepton from each causes the event to trigger). Assuming $\epsilon_{2}=1-\left(1-\epsilon_{1}\right)^{2}$, which is conservative since it minimizes the value of $\mu_{S}$, transforms Eq. (1) into

$$
\mu_{S}=2 \mathcal{L} \sigma \mathcal{B} \epsilon_{1}\left[1-\frac{1}{2} \mathcal{B} \epsilon_{1}\right](1-f) .
$$

Since $\mu_{S}$ in Eq. (2) depends not only on $\sigma \mathcal{B}$, but also on $\mathcal{B}$, the upper limits on $\sigma \mathcal{B}$ depend on the assumed value of $\mathcal{B}$, scaling approximately as the expression $1 /\left[1-\frac{1}{2} \mathcal{B} \epsilon_{1}\right]$. The upper limits are thus best for low values of $\mathcal{B}$, though the dependence of the limits on $\mathcal{B}$ is weak, particularly if $\epsilon_{1}$ is small. We set the value of $\mathcal{B}$ equal to unity in the expression in square brackets, so as to obtain conservative limits that are valid for any value of $\mathcal{B}$.

For each combination of the $H$ and $X$ boson masses that is modeled, and for a range of mean proper decay lengths $c \tau$ of the $X$ boson, 95\% C.L. upper limits on
$\sigma(H \rightarrow X X) \mathcal{B}\left(X \rightarrow \ell^{+} \ell^{-}\right)$are calculated. The observed limits for the electron and muon channels are shown in Figs. 4 and 5, respectively. The less stringent limits for the muon channel in the $m_{H}=1000 \mathrm{GeV} / c^{2}, m_{X}=$ $20 \mathrm{GeV} / c^{2}$ case are caused by low trigger efficiency for nearby muons, and the consequent $\Delta R$ requirement. The corresponding limits on $\sigma\left(\tilde{q} \tilde{q}^{*}+\tilde{q} \tilde{q}\right) \mathcal{B}\left(\tilde{q} \rightarrow q \tilde{\chi}^{0}\right.$, $\left.\tilde{\chi}^{0} \rightarrow \ell^{+} \ell^{-} \nu\right)$ are shown in Fig. 6. The shaded band in each of these plots shows the $\pm 1 \sigma$ range of variation of the expected $95 \%$ C.L. limits, illustrated for one choice of masses. All the observed limits are consistent with the corresponding expected ones.

At $\sqrt{s}=8 \mathrm{TeV}$, the theoretical cross sections for SM Higgs boson production through the dominant gluon-gluon fusion mechanism are 19.3, 7.1, 2.9, and $0.03 \mathrm{pb}$ for Higgs boson masses of $125,200,400$, and $1000 \mathrm{GeV} / c^{2}$, respectively [30]. The theoretical cross sections for $\tilde{q} \tilde{q}^{*}+$ $\tilde{q} \tilde{q}$ production are $2590,10,0.014$, and 0.00067 pb for $\tilde{q}$ masses of $120,350,1000$, and $1500 \mathrm{GeV} / c^{2}$, as evaluated with the PROSPINO generator [31] assuming a gluino mass of $5 \mathrm{TeV} / c^{2}$. The observed limits on $\sigma \mathcal{B}$ are usually well below these theoretical cross sections, implying that nontrivial bounds are being placed on the decay modes involving LL particles, probing, for example, branching fractions as low as $10^{-4}$ and $10^{-6}$ in the Higgs and supersymmetric models, respectively.

We also compute upper limits on the cross section times branching fraction within the acceptance $A$, where the latter is defined in the last paragraph of Sec. IV. Figures 7-8 show for the electron and muon channels, respectively, these limits on $\sigma(H \rightarrow X X) \mathcal{B}\left(X \rightarrow \ell^{+} \ell^{-}\right) A\left(X \rightarrow \ell^{+} \ell^{-}\right)$. 

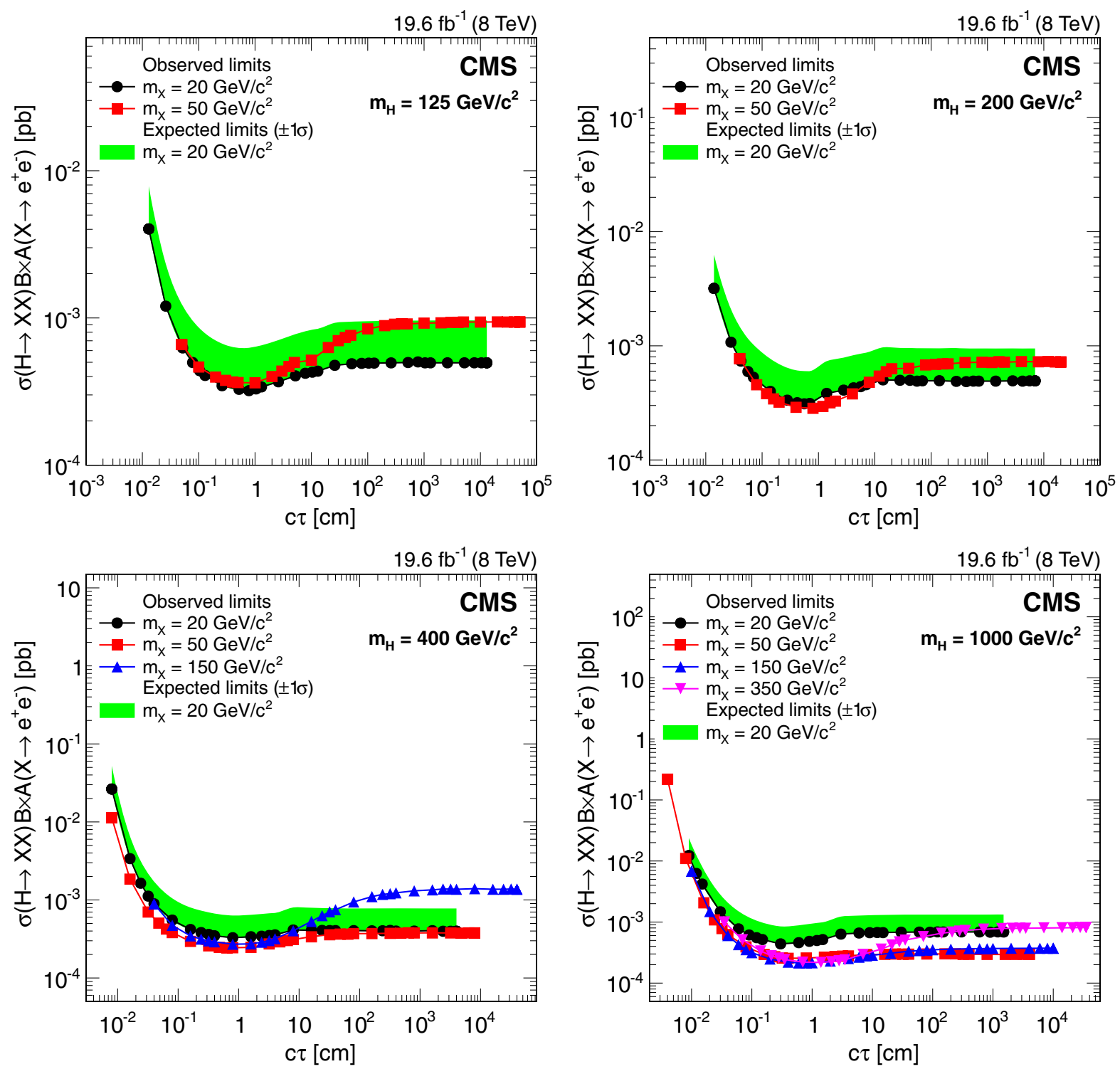

FIG. 7 (color online). The 95\% C.L. upper limits on $\sigma(H \rightarrow X X) \mathcal{B}\left(X \rightarrow e^{+} e^{-}\right) A\left(X \rightarrow e^{+} e^{-}\right)$, as a function of the mean proper decay length of the $X$ boson, for Higgs boson masses of 125 (top left), 200 (top right), 400 (bottom left), and $1000 \mathrm{GeV} / c^{2}$ (bottom right). In each plot, results are shown for several $X$ boson mass hypotheses. The shaded band shows the $\pm 1 \sigma$ range of variation of the expected $95 \%$ C.L. limits for the case of a $20 \mathrm{GeV} / c^{2} X$ boson mass. Corresponding bands for the other $X$ boson masses, omitted for clarity of presentation, show similar agreement with the respective observed limits.

Figure 9 shows the corresponding limits on $\sigma\left(\tilde{q} \tilde{q}^{*}+\right.$ $\tilde{q} \tilde{q}) \mathcal{B}\left(\tilde{q} \rightarrow q \tilde{\chi}^{0}, \tilde{\chi}^{0} \rightarrow \ell^{+} \ell^{-} \nu\right) A\left(\tilde{q} \rightarrow q \tilde{\chi}^{0}, \tilde{\chi}^{0} \rightarrow \ell^{+} \ell^{-} \nu\right)$. These limits restricted to the acceptance region show substantially less dependence on the Higgs boson and $X$ boson masses and on the mean proper decay length $c \tau$ of the $X$ boson. They are also less model dependent, as can be seen by the fact that the limits on $\sigma \mathcal{B} A$ are similar for $X \rightarrow$ $\ell^{+} \ell^{-}$and $\tilde{\chi}^{0} \rightarrow \ell^{+} \ell^{-} \nu$. The residual dependence of the limits on $c \tau$ is due to the $\left|d_{0}\right| / \sigma_{d}>12$ requirement at small values of $c \tau$; whereas at larger values of $c \tau$, it is caused by the fact that, even within the defined acceptance region, the tracking efficiency falls for leptons produced far from the beam line with very large impact parameters.
Although the limits described above are determined in the context of two specific models, the analysis is sensitive to any process in which an LL particle is produced and subsequently decays to a final state that includes dileptons. To place approximate limits on this more general class of models, one should use the limits within the acceptance region (i.e. on $\sigma \mathcal{B A}$ ), because of their smaller model dependence. In most signal models in which each event contains two identical LL particles that decay in this way, the limits on $\sigma \mathcal{B} A$ shown in Figs. 7-9 should remain approximately valid. (The variation among the limit curves shown in these plots for different signal models and particle masses gives an indication of the accuracy of this 

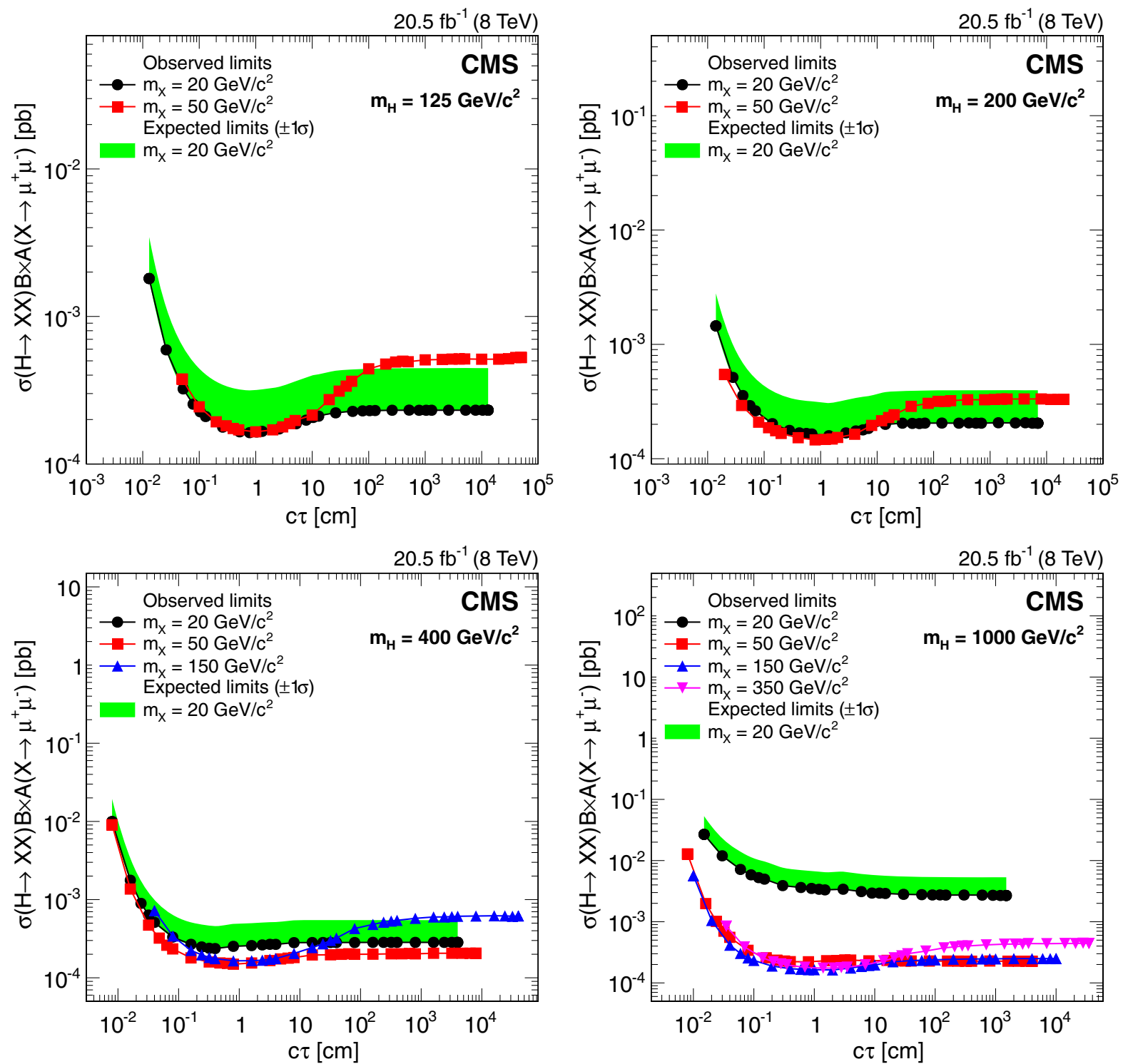

FIG. 8 (color online). The 95\% C.L. upper limits on $\sigma(H \rightarrow X X) \mathcal{B}\left(X \rightarrow \mu^{+} \mu^{-}\right) A\left(X \rightarrow \mu^{+} \mu^{-}\right)$, as a function of the mean proper decay length of the $X$ boson, for Higgs boson masses of 125 (top left), 200 (top right), 400 (bottom left), and $1000 \mathrm{GeV} / c^{2}$ (bottom right). In each plot, results are shown for several $X$ boson mass hypotheses. The shaded band shows the $\pm 1 \sigma$ range of variation of the expected $95 \%$ C.L. limits for the case of a $20 \mathrm{GeV} / c^{2} X$ boson mass. Corresponding bands for the other $X$ boson masses, omitted for clarity of presentation, show similar agreement with the respective observed limits.

statement.) Exceptions could arise for models that give poor efficiency within the acceptance criteria, e.g. for models in which the leptons are not isolated; have impact parameters with significance below $\left|d_{0}\right| / \sigma_{d}<12$, corresponding to $\left|d_{0}\right| \lesssim 180 \mu \mathrm{m}$; are almost collinear with each other (with the dilepton mass below $15 \mathrm{GeV} / c^{2}$, or for the muon channel $\Delta R<0.2$ ); or do not usually satisfy the $|\Delta \Phi|<\pi / 2$ criterion, such that the parameter $f$ becomes large (e.g. if the LL particle is slow moving and decays to many particles).

In models where each event contains only one LL particle that can decay inclusively to dileptons, the expected number of selected signal events for given $\sigma \mathcal{B}$ will be up to a factor of two lower, and so the limits on $\sigma \mathcal{B A}$ will be up to a factor of two worse than those shown in Figs. 7-9.

The acceptance $A$ for any given model can be determined with a generator-level simulation, allowing limits on $\sigma \mathcal{B A}$ to be converted to limits on $\sigma \mathcal{B}$. The following example illustrates this. The limits on $\sigma(H \rightarrow X X) \mathcal{B}\left(X \rightarrow \ell^{+} \ell^{-}\right)$ quoted above are for $H$ bosons produced through gluongluon fusion. If the $H$ bosons were instead produced by the sum of all SM production mechanisms, their momentum spectra would be slightly harder. For $m_{H}=125 \mathrm{GeV} / c^{2}$, the acceptance would then be larger by a factor of approximately $1.18(1.12)$ for $m_{X}=20(50) \mathrm{GeV} / c^{2}$, with 

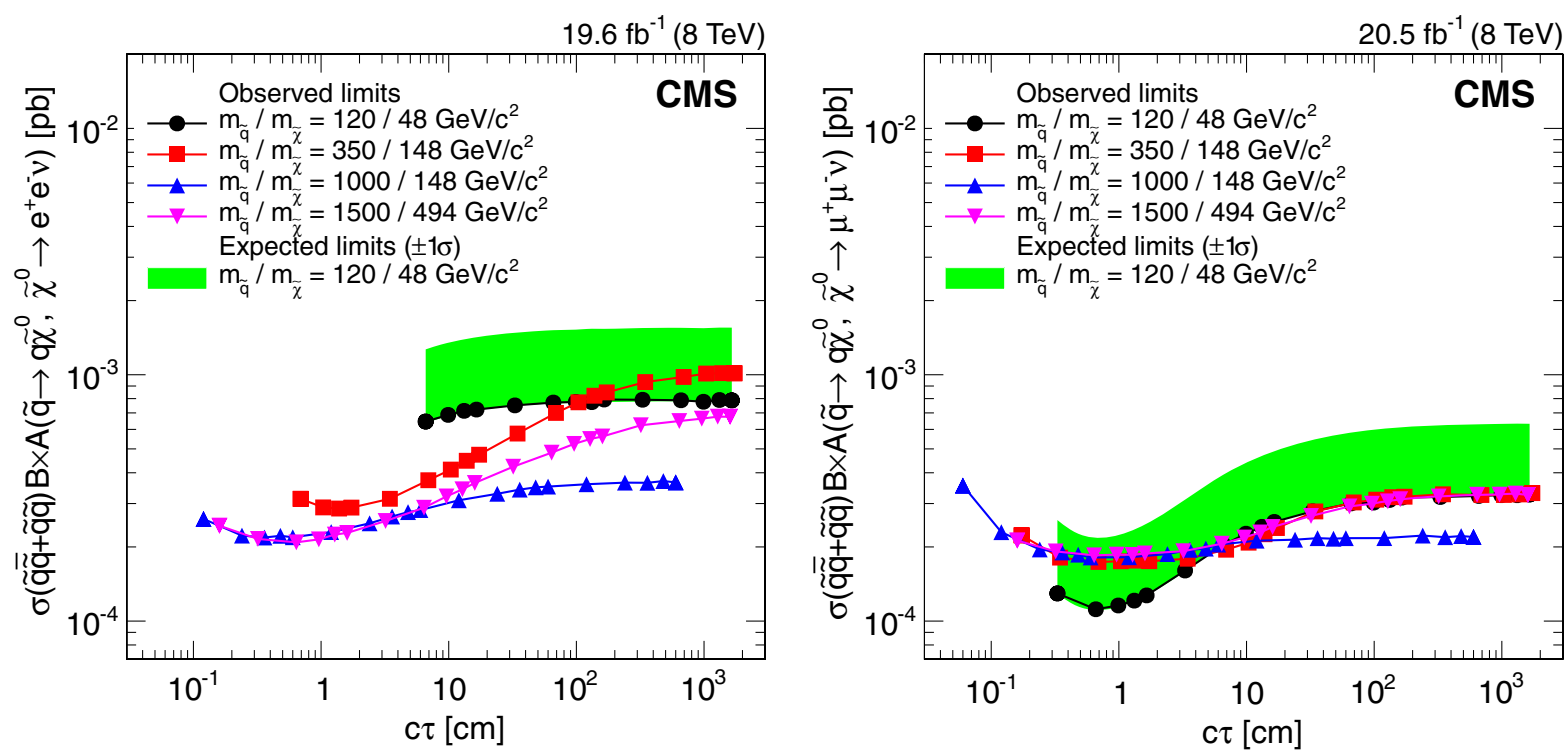

FIG. 9 (color online). The 95\% C.L. upper limits on $\sigma\left(\tilde{q} \tilde{q}^{*}+\tilde{q} \tilde{q}\right) \mathcal{B}\left(\tilde{q} \rightarrow q \tilde{\chi}^{0}, \tilde{\chi}^{0} \rightarrow \ell^{+} \ell^{-} \nu\right) A\left(\tilde{q} \rightarrow q \tilde{\chi}^{0}, \tilde{\chi}^{0} \rightarrow \ell^{+} \ell^{-} \nu\right)$ for the electron (left), and muon (right) channels, as a function of the mean proper decay length of the neutralino. The shaded band shows the $\pm 1 \sigma$ range of variation of the expected $95 \%$ C.L. limits for the case of a $120 \mathrm{GeV} / c^{2}$ squark and a $48 \mathrm{GeV} / c^{2}$ neutralino mass. Corresponding bands for the other squark and neutralino masses, omitted for clarity of presentation, show similar agreement with the respective observed limits.

a corresponding improvement in the limits on $\sigma \mathcal{B}$. The change is smaller for larger $H$ boson masses.

\section{SUMMARY}

A search has been performed, using proton-proton collision data collected at $\sqrt{s}=8 \mathrm{TeV}$, for long-lived particles that decay to a final state that includes a pair of electrons or a pair of muons. No such events have been seen. Quantitative limits have been placed on the product of the cross section and branching fraction of such a signal in the context of two specific models. In the first model, a Higgs boson, in the mass range $125-1000 \mathrm{GeV} / c^{2}$, decays into a pair of hypothetical, long-lived neutral bosons in the mass range $20-350 \mathrm{GeV} / c^{2}$, each of which can decay to dileptons. The upper limits obtained are typically in the range $0.2-10 \mathrm{fb}$ for long-lived particles with mean proper decay lengths in the range $0.01-100 \mathrm{~cm}$, and weaken to $250 \mathrm{fb}$ for the lowest considered Higgs mass of $125 \mathrm{GeV} / c^{2}$. In the second model, based on R-parity violating supersymmetry, a pair of squarks each decays to a quark and a long-lived neutralino $\tilde{\chi}^{0}$; the neutralino can subsequently decay to $e^{+} e^{-} \nu$ or $\mu^{+} \mu^{-} \nu$. In this case, the upper limits are typically in the range $0.2-5 \mathrm{fb}$ for $\tilde{\chi}^{0}$ mean proper decay lengths in the range $0.1-100 \mathrm{~cm}$ and squark masses above $350 \mathrm{GeV} / c^{2}$. For a lower squark mass of $120 \mathrm{GeV} / c^{2}$, the limits are typically a factor of ten weaker. These limits are sensitive to branching fractions as low as $10^{-4}$ and $10^{-6}$ in the Higgs boson and supersymmetric models, respectively. To allow the results to be reinterpreted in the context of other models, limits that are restricted to the detector acceptance are also presented, reducing the model dependence. Over much of the investigated parameter space, these limits are the most stringent to date.

\section{ACKNOWLEDGMENTS}

We congratulate our colleagues in the CERN accelerator departments for the excellent performance of the LHC and thank the technical and administrative staffs at CERN and at other CMS institutes for their contributions to the success of the CMS effort. In addition, we gratefully acknowledge the computing centers and personnel of the Worldwide LHC Computing Grid for delivering so effectively the computing infrastructure essential to our analyses. Finally, we acknowledge the enduring support for the construction and operation of the LHC and the CMS detector provided by the following funding agencies: BMWFW and FWF (Austria); FNRS and FWO (Belgium); CNPq, CAPES, FAPERJ, and FAPESP (Brazil); MES (Bulgaria); CERN; CAS, MoST, and NSFC (China); COLCIENCIAS (Colombia); MSES and CSF (Croatia); RPF (Cyprus); MoER, ERC IUT and ERDF (Estonia); Academy of Finland, MEC, and HIP (Finland); CEA and CNRS/ IN2P3 (France); BMBF, DFG, and HGF (Germany); GSRT (Greece); OTKA and NIH (Hungary); DAE and DST (India); IPM (Iran); SFI (Ireland); INFN (Italy); MSIP and NRF (Republic of Korea); LAS (Lithuania); MOE and UM (Malaysia); CINVESTAV, CONACYT, SEP, and UASLP-FAI (Mexico); MBIE (New Zealand); PAEC (Pakistan); MSHE and NSC (Poland); FCT (Portugal); JINR (Dubna); MON, RosAtom, RAS and RFBR (Russia); 
MESTD (Serbia); SEIDI and CPAN (Spain); Swiss Funding Agencies (Switzerland); MST (Taipei); ThEPCenter, IPST, STAR and NSTDA (Thailand); TUBITAK and TAEK (Turkey); NASU and SFFR (Ukraine); STFC (United Kingdom); DOE and NSF (USA). Individuals have received support from the Marie Curie program and the European Research Council and EPLANET (European Union); the Leventis Foundation; the A. P. Sloan Foundation; the Alexander von Humboldt Foundation; the Belgian Federal Science Policy Office; the Fonds pour la Formation à la Recherche dans l'Industrie et dans l'Agriculture (FRIA Belgium); the
Agentschap voor Innovatie door Wetenschap en Technologie (IWT Belgium); the Ministry of Education, Youth and Sports (MEYS) of the Czech Republic; the Council of Science and Industrial Research, India; the HOMING PLUS program of Foundation for Polish Science, cofinanced by the European Union, Regional Development Fund; the Compagnia di San Paolo (Torino); the Consorzio per la Fisica (Trieste); MIUR Grant No. 20108T4XTM (Italy); the Thalis and Aristeia programmes cofinanced by EU-ESF and the Greek NSRF; and the National Priorities Research Program by Qatar National Research Fund.
[1] J. L. Hewett, B. Lillie, M. Masip, and T. G. Rizzo, Signatures of long-lived gluinos in split supersymmetry, J. High Energy Phys. 09 (2004) 070.

[2] R. Barbier, C. Bérat, M. Besançon, M. Chemtob, A. Deandrea, E. Dudas, P. Fayet, S. Lavignac, G. Moreau, E. Perez, and Y. Sirois, $R$-parity violating supersymmetry, Phys. Rep. 420, 1 (2005).

[3] T. Han, Z. Si, K. M. Zurek, and M. J. Strassler, Phenomenology of hidden valleys at hadron colliders, J. High Energy Phys. 07 (2008) 008.

[4] L. Basso, A. Belyaev, S. Moretti, and C. H. ShepherdThemistocleous, Phenomenology of the minimal $B-L$ extension of the standard model: $Z^{\prime}$ and neutrinos, Phys. Rev. D 80, 055030 (2009).

[5] M. J. Strassler and K. M. Zurek, Discovering the Higgs through highly-displaced vertices, Phys. Lett. B 661, 263 (2008).

[6] B. C. Allanach, M. A. Bernhardt, H. K. Dreiner, C. H. Kom, and P. Richardson, Mass spectrum in $R$-parity violating minimal supergravity and benchmark points, Phys. Rev. D 75, 035002 (2007).

[7] CMS Collaboration, Search in leptonic channels for heavy resonances decaying to long-lived neutral particles, J. High Energy Phys. 02 (2013) 085.

[8] CMS Collaboration, Search for Displaced Supersymmetry in Events with an Electron and a Muon with Large Impact Parameters, Phys. Rev. Lett. 114, 061801 (2015).

[9] CMS Collaboration, Search for long-lived neutral particles decaying to dijets, Phys. Rev. D 91, 012007 (2015).

[10] V. M. Abazov et al. (D0 Collaboration), Search for Neutral, Long-Lived Particles Decaying into Two Muons in $p \bar{p}$ Collisions at $\sqrt{s}=1.96 \mathrm{TeV}$, Phys. Rev. Lett. 97, 161802 (2006).

[11] V. M. Abazov et al. (D0 Collaboration), Search for LongLived Particles Decaying into Electron or Photon Pairs with the D0 Detector, Phys. Rev. Lett. 101, 111802 (2008).

[12] ATLAS Collaboration, Search for A Light Higgs Boson Decaying to Long-Lived Weakly-Interacting Particles in Proton-Proton Collisions at $\sqrt{s}=7 \mathrm{TeV}$ with the ATLAS Detector, Phys. Rev. Lett. 108, 251801 (2012).
[13] ATLAS Collaboration, Search for long-lived, heavy particles in final states with a muon and multi-track displaced vertex in proton-proton collisions at $\sqrt{s}=7 \mathrm{TeV}$ with the ATLAS detector, Phys. Lett. B 719, 280 (2013).

[14] ATLAS Collaboration, Search for long-lived neutral particles decaying into lepton jets in proton-proton collisions at $\sqrt{s}=8 \mathrm{TeV}$ with the ATLAS detector, J. High Energy Phys. 11 (2014) 088.

[15] CMS Collaboration, The CMS experiment at the CERN LHC, J. Instrum. 3, S08004 (2008).

[16] S. Chatrchyan et al. (CMS Collaboration), Description and performance of track and primary-vertex reconstruction with the CMS tracker, J. Instrum. 9, P10009 (2014).

[17] CMS Collaboration, CMS tracking performance results from early LHC operation, Eur. Phys. J. C 70, 1165 (2010).

[18] CMS Collaboration, Energy calibration and resolution of the CMS electromagnetic calorimeter in pp collisions at $\sqrt{s}=7 \mathrm{TeV}$, J. Instrum. 8, P09009 (2013).

[19] CMS Collaboration, Observation of the diphoton decay of the Higgs boson and measurement of its properties, Eur. Phys. J. C 74, 3076 (2014).

[20] T. Sjöstrand, S. Mrenna, and P. Z. Skands, PYTHIA 6.4 physics and manual, J. High Energy Phys. 05 (2006) 026.

[21] S. Agostinelli et al. (GEANT4 Collaboration), GEANT4-a simulation toolkit, Nucl. Instrum. Methods Phys. Res., Sect. A 506, 250 (2003).

[22] CMS Collaboration, CMS luminosity based on pixel cluster counting-Summer 2013 update, Report No. CMS-PASLUM-13-001, 2013, http://cdsweb.cern.ch/record/1598864.

[23] D. Bourilkov, R. C. Group, and M. R. Whalley, LHAPDF: PDF use from the Tevatron to the LHC, arXiv:hep-ph/ 0605240

[24] CMS Collaboration, Studies of tracker material, Report No. CMS-PAS-TRK-10-003, 2010, http://cdsweb.cern.ch/ record/1279138.

[25] CMS Collaboration, Measurements of inclusive $\mathrm{W}$ and $\mathrm{Z}$ cross sections in pp collisions at $\sqrt{s}=7 \mathrm{TeV}$, J. High Energy Phys. 01 (2011) 080.

[26] P. Nason, A new method for combining NLO QCD with shower Monte Carlo algorithms, J. High Energy Phys. 11 (2004) 040. 
[27] S. Frixione, P. Nason, and C. Oleari, Matching NLO QCD computations with parton shower simulations: the POWHEG method, J. High Energy Phys. 11 (2007) 070.

[28] S. Alioli, P. Nason, C. Oleari, and E. Re, A general framework for implementing NLO calculations in shower Monte Carlo programs: the POWHEG BOX, J. High Energy Phys. 06 (2010) 043.

[29] ATLAS Collaboration, CMS Collaboration, and LHC Higgs Combination Group, Procedure for the LHC Higgs boson search combination in Summer 2011, Reports No. ATLPHYS-PUB-2011-011 and No. CMS-NOTE-2011-005, 2011, http://cdsweb.cern.ch/record/1598864.

[30] S. Heinemeyer et al., Handbook of LHC Higgs cross sections: 3. Higgs properties, CERN Report No. CERN-2013004, 2013, http://dx.doi.org/10.5170/CERN-2013-004.

[31] W. Beenakker, R. Höpker, M. Spira, and P. M. Zerwas, Squark and gluino production at hadron colliders, Nucl. Phys. B492, 51 (1997).

V. Khachatryan, ${ }^{1}$ A. M. Sirunyan, ${ }^{1}$ A. Tumasyan, ${ }^{1}$ W. Adam, ${ }^{2}$ T. Bergauer, ${ }^{2}$ M. Dragicevic, ${ }^{2}$ J. Erö, ${ }^{2}$ M. Friedl, ${ }^{2}$ R. Frühwirth, ${ }^{2, b}$ V. M. Ghete, ${ }^{2}$ C. Hartl, ${ }^{2}$ N. Hörmann, ${ }^{2}$ J. Hrubec, ${ }^{2}$ M. Jeitler, ${ }^{2, b}$ W. Kiesenhofer, ${ }^{2}$ V. Knünz, ${ }^{2}$ M. Krammer, ${ }^{2, b}$ I. Krätschmer, ${ }^{2}$ D. Liko, ${ }^{2}$ I. Mikulec, ${ }^{2}$ D. Rabady, ${ }^{2, \mathrm{c}}$ B. Rahbaran, ${ }^{2}$ H. Rohringer, ${ }^{2}$ R. Schöfbeck, ${ }^{2}$ J. Strauss, ${ }^{2}$ W. Treberer-Treberspurg, ${ }^{2}$ W. Waltenberger, ${ }^{2}$ C.-E. Wulz, ${ }^{2, b}$ V. Mossolov, ${ }^{3}$ N. Shumeiko, ${ }^{3}$ J. Suarez Gonzalez, ${ }^{3}$ S. Alderweireldt, ${ }^{4}$ M. Bansal, ${ }^{4}$ S. Bansal, ${ }^{4}$ T. Cornelis, ${ }^{4}$ E. A. De Wolf, ${ }^{4}$ X. Janssen, ${ }^{4}$ A. Knutsson, ${ }^{4}$ J. Lauwers, ${ }^{4}$ S. Luyckx, ${ }^{4}$

S. Ochesanu, ${ }^{4}$ R. Rougny, ${ }^{4}$ M. Van De Klundert, ${ }^{4}$ H. Van Haevermaet, ${ }^{4}$ P. Van Mechelen, ${ }^{4}$ N. Van Remortel, ${ }^{4}$ A. Van Spilbeeck, ${ }^{4}$ F. Blekman, ${ }^{5}$ S. Blyweert, ${ }^{5}$ J. D'Hondt, ${ }^{5}$ N. Daci, ${ }^{5}$ N. Heracleous, ${ }^{5}$ J. Keaveney, ${ }^{5}$ S. Lowette, ${ }^{5}$ M. Maes, ${ }^{5}$ A. Olbrechts, ${ }^{5}$ Q. Python, ${ }^{5}$ D. Strom, ${ }^{5}$ S. Tavernier, ${ }^{5}$ W. Van Doninck, ${ }^{5}$ P. Van Mulders, ${ }^{5}$ G. P. Van Onsem, ${ }^{5}$ I. Villella, ${ }^{5}$ C. Caillol, ${ }^{6}$ B. Clerbaux, ${ }^{6}$ G. De Lentdecker, ${ }^{6}$ D. Dobur, ${ }^{6}$ L. Favart, ${ }^{6}$ A. P. R. Gay, ${ }^{6}$ A. Grebenyuk, ${ }^{6}$ A. Léonard, ${ }^{6}$ A. Mohammadi, ${ }^{6}$ L. Perniè, ${ }^{6, c}$ A. Randle-conde, ${ }^{6}$ T. Reis, ${ }^{6}$ T. Seva, ${ }^{6}$ L. Thomas, ${ }^{6}$ C. Vander Velde, ${ }^{6}$ P. Vanlaer, ${ }^{6}$ J. Wang, ${ }^{6}$ F. Zenoni, ${ }^{6}$ V. Adler, ${ }^{7}$ K. Beernaert, ${ }^{7}$ L. Benucci, ${ }^{7}$ A. Cimmino, ${ }^{7}$ S. Costantini, ${ }^{7}$ S. Crucy, ${ }^{7}$ S. Dildick, ${ }^{7}$ A. Fagot, ${ }^{7}$ G. Garcia, ${ }^{7}$ J. Mccartin, ${ }^{7}$ A. A. Ocampo Rios, ${ }^{7}$ D. Ryckbosch, ${ }^{7}$ S. Salva Diblen, ${ }^{7}$ M. Sigamani, ${ }^{7}$ N. Strobbe, ${ }^{7}$ F. Thyssen, ${ }^{7}$ M. Tytgat, ${ }^{7}$ E. Yazgan, ${ }^{7}$ N. Zaganidis, ${ }^{7}$ S. Basegmez, ${ }^{8}$ C. Beluffi, ${ }^{8, d}$ G. Bruno, ${ }^{8}$ R. Castello, ${ }^{8}$ A. Caudron, ${ }^{8}$ L. Ceard, ${ }^{8}$ G. G. Da Silveira, ${ }^{8}$ C. Delaere, ${ }^{8}$ T. du Pree, ${ }^{8}$ D. Favart, ${ }^{8}$ L. Forthomme, ${ }^{8}$ A. Giammanco, ${ }^{8, e}$ J. Hollar, ${ }^{8}$ A. Jafari, ${ }^{8}$ P. Jez, ${ }^{8}$ M. Komm, ${ }^{8}$ V. Lemaitre, ${ }^{8}$ C. Nuttens, ${ }^{8}$ D. Pagano, ${ }^{8}$ L. Perrini, ${ }^{8}$ A. Pin, ${ }^{8}$ K. Piotrzkowski, ${ }^{8}$ A. Popov, ${ }^{8, f}$ L. Quertenmont, ${ }^{8}$ M. Selvaggi, ${ }^{8}$ M. Vidal Marono, ${ }^{8}$ J. M. Vizan Garcia,${ }^{8}$ N. Beliy, ${ }^{9}$ T. Caebergs, ${ }^{9}$ E. Daubie, ${ }^{9}$ G. H. Hammad, ${ }^{9}$ W. L. Aldá Júnior, ${ }^{10}$ G. A. Alves, ${ }^{10}$ L. Brito, ${ }^{10}$ M. Correa Martins Junior, ${ }^{10}$ T. Dos Reis Martins,${ }^{10}$ C. Mora Herrera, ${ }^{10}$ M. E. Pol, ${ }^{10}$ P. Rebello Teles, ${ }^{10}$ W. Carvalho, ${ }^{11}$ J. Chinellato, ${ }^{11, \mathrm{~g}}$ A. Custódio, ${ }^{11}$ E. M. Da Costa, ${ }^{11}$ D. De Jesus Damiao, ${ }^{11}$ C. De Oliveira Martins, ${ }^{11}$ S. Fonseca De Souza, ${ }^{11}$ H. Malbouisson, ${ }^{11}$ D. Matos Figueiredo, ${ }^{11}$ L. Mundim, ${ }^{11}$ H. Nogima,${ }^{11}$ W. L. Prado Da Silva, ${ }^{11}$ J. Santaolalla, ${ }^{11}$ A. Santoro, ${ }^{11}$ A. Sznajder, ${ }^{11}$ E. J. Tonelli Manganote, ${ }^{11, g}$ A. Vilela Pereira, ${ }^{11}$ C. A. Bernardes, ${ }^{12 b}$ S. Dogra, ${ }^{12 a}$ T. R. Fernandez Perez Tomei, ${ }^{12 a}$ E. M. Gregores, ${ }^{12 b}$ P. G. Mercadante, ${ }^{12 b}$ S. F. Novaes, ${ }^{12 a}$ Sandra S. Padula, ${ }^{12 a}$ A. Aleksandrov, ${ }^{13}$ V. Genchev, ${ }^{13, c}$ R. Hadjiiska, ${ }^{13}$ P. Iaydjiev, ${ }^{13}$ A. Marinov, ${ }^{13}$ S. Piperov, ${ }^{13}$ M. Rodozov, ${ }^{13}$ G. Sultanov, ${ }^{13}$ M. Vutova, ${ }^{13}$ A. Dimitrov, ${ }^{14}$ I. Glushkov, ${ }^{14}$ L. Litov, ${ }^{14}$ B. Pavlov, ${ }^{14}$ P. Petkov, ${ }^{14}$ J. G. Bian, ${ }^{15}$ G. M. Chen, ${ }^{15}$ H. S. Chen, ${ }^{15}$ M. Chen, ${ }^{15}$ T. Cheng, ${ }^{15}$ R. Du, ${ }^{15}$ C. H. Jiang, ${ }^{15}$ R. Plestina,${ }^{15}$ h. F. Romeo, ${ }^{15}$ J. Tao, ${ }^{15}$ Z. Wang, ${ }^{15}$ C. Asawatangtrakuldee,${ }^{16}$ Y. Ban,${ }^{16}$ Q. Li ${ }^{16}$ S. Liu, ${ }^{16}$ Y. Mao, ${ }^{16}$ S. J. Qian, ${ }^{16}$ D. Wang, ${ }^{16}$ W. Zou, ${ }^{16}$ C. Avila,${ }^{17}$ A. Cabrera, ${ }^{17}$ L. F. Chaparro Sierra,${ }^{17}$ C. Florez,${ }^{17}$ J. P. Gomez,${ }^{17}$ B. Gomez Moreno, ${ }^{17}$ J. C. Sanabria,${ }^{17}$ N. Godinovic,${ }^{18}$ D. Lelas, ${ }^{18}$ D. Polic, ${ }^{18}$ I. Puljak, ${ }^{18}$ Z. Antunovic, ${ }^{19}$ M. Kovac, ${ }^{19}$ V. Brigljevic, ${ }^{20}$ K. Kadija,${ }^{20}$ J. Luetic,${ }^{20}$ D. Mekterovic,${ }^{20}$ L. Sudic,${ }^{20}$ A. Attikis, ${ }^{21}$ G. Mavromanolakis ${ }^{21}$ J. Mousa, ${ }^{21}$ C. Nicolaou, ${ }^{21}$ F. Ptochos,${ }^{21}$ P. A. Razis, ${ }^{21}$ M. Bodlak,${ }^{22}$ M. Finger, ${ }^{22}$ M. Finger Jr., ${ }^{22, \mathrm{i}}$ Y. Assran, ${ }^{23, \mathrm{j}}$ S. Elgammal, ${ }^{23, \mathrm{k}}$ M. A. Mahmoud, ${ }^{23,1}$ A. Radi, ${ }^{23, \mathrm{~m}, \mathrm{n}}$ M. Kadastik ${ }^{24}$ M. Murumaa, ${ }^{24}$ M. Raidal, ${ }^{24}$ A. Tiko, ${ }^{24}$ P. Eerola, ${ }^{25}$ G. Fedi, ${ }^{25}$ M. Voutilainen, ${ }^{25}$ J. Härkönen, ${ }^{26}$ V. Karimäki,${ }^{26}$ R. Kinnunen, ${ }^{26}$ M. J. Kortelainen, ${ }^{26}$ T. Lampén, ${ }^{26}$ K. Lassila-Perini, ${ }^{26}$ S. Lehti, ${ }^{26}$ T. Lindén, ${ }^{26}$ P. Luukka, ${ }^{26}$ T. Mäenpääa ${ }^{26}$ T. Peltola, ${ }^{26}$ E. Tuominen, ${ }^{26}$ J. Tuominiemi, ${ }^{26}$ E. Tuovinen, ${ }^{26}$ L. Wendland, ${ }^{26}$ J. Talvitie, ${ }^{27}$ T. Tuuva, ${ }^{27}$ M. Besancon, ${ }^{28}$ F. Couderc, ${ }^{28}$ M. Dejardin, ${ }^{28}$ D. Denegri, ${ }^{28}$ B. Fabbro, ${ }^{28}$ J. L. Faure, ${ }^{28}$ C. Favaro, ${ }^{28}$ F. Ferri, ${ }^{28}$ S. Ganjour, ${ }^{28}$ A. Givernaud,${ }^{28}$ P. Gras,${ }^{28}$ G. Hamel de Monchenault, ${ }^{28}$ P. Jarry, ${ }^{28}$ E. Locci, ${ }^{28}$ J. Malcles,${ }^{28}$ J. Rander ${ }^{28}$ A. Rosowsky, ${ }^{28}$ M. Titov,${ }^{28}$ S. Baffioni, ${ }^{29}$ F. Beaudette, ${ }^{29}$ P. Busson, ${ }^{29}$ C. Charlot, ${ }^{29}$ T. Dahms, ${ }^{29}$ M. Dalchenko, ${ }^{29}$ L. Dobrzynski, ${ }^{29}$ N. Filipovic,${ }^{29}$ A. Florent, ${ }^{29}$ R. Granier de Cassagnac, ${ }^{29}$ L. Mastrolorenzo, ${ }^{29}$ P. Miné, ${ }^{29}$ C. Mironov, ${ }^{29}$ I. N. Naranjo, ${ }^{29}$ M. Nguyen, ${ }^{29}$ C. Ochando, ${ }^{29}$ P. Paganini ${ }^{29}$ S. Regnard, ${ }^{29}$ R. Salerno,${ }^{29}$ J. B. Sauvan, ${ }^{29}$ Y. Sirois,${ }^{29}$ C. Veelken, ${ }^{29}$ Y. Yilmaz,${ }^{29}$ A. Zabi, ${ }^{29}$ J.-L. Agram, ${ }^{30,0}$ J. Andrea, ${ }^{30}$ A. Aubin, ${ }^{30}$ D. Bloch,${ }^{30}$ J.-M. Brom,${ }^{30}$ E. C. Chabert,${ }^{30}$ C. Collard ${ }^{30}$ E. Conte, ${ }^{30,0}$ J.-C. Fontaine,${ }^{30,0}$ D. Gelé, ${ }^{30}$ 
U. Goerlach ${ }^{30}$ C. Goetzmann, ${ }^{30}$ A.-C. Le Bihan, ${ }^{30}$ K. Skovpen, ${ }^{30}$ P. Van Hove,${ }^{30}$ S. Gadrat,${ }^{31}$ S. Beauceron, ${ }^{32}$ N. Beaupere, ${ }^{32}$ G. Boudoul, ${ }^{32, \mathrm{c}}$ E. Bouvier, ${ }^{32}$ S. Brochet,${ }^{32}$ C. A. Carrillo Montoya, ${ }^{32}$ J. Chasserat, ${ }^{32}$ R. Chierici, ${ }^{32}$ D. Contardo, ${ }^{32, \mathrm{c}}$ P. Depasse, ${ }^{32}$ H. El Mamouni, ${ }^{32}$ J. Fan, ${ }^{32}$ J. Fay, ${ }^{32}$ S. Gascon,${ }^{32}$ M. Gouzevitch, ${ }^{32}$ B. Ille, ${ }^{32}$ T. Kurca, ${ }^{32}$ M. Lethuillier, ${ }^{32}$ L. Mirabito, ${ }^{32}$ S. Perries, ${ }^{32}$ J. D. Ruiz Alvarez, ${ }^{32}$ D. Sabes,${ }^{32}$ L. Sgandurra, ${ }^{32}$ V. Sordini, ${ }^{32}$ M. Vander Donckt, ${ }^{32}$ P. Verdier, ${ }^{32}$ S. Viret ${ }^{32}$ H. Xiao, ${ }^{32}$ Z. Tsamalaidze, ${ }^{33, i}$ C. Autermann, ${ }^{34}$ S. Beranek,${ }^{34}$ M. Bontenackels, ${ }^{34}$ M. Edelhoff, ${ }^{34}$ L. Feld,${ }^{34}$ A. Heister, ${ }^{34}$ O. Hindrichs,${ }^{34}$ K. Klein, ${ }^{34}$ A. Ostapchuk, ${ }^{34}$ F. Raupach, ${ }^{34}$ J. Sammet,${ }^{34}$ S. Schael,${ }^{34}$ H. Weber ${ }^{34}$ B. Wittmer,${ }^{34}$ V. Zhukov, ${ }^{34, f}$ M. Ata,${ }^{35}$ M. Brodski, ${ }^{35}$ E. Dietz-Laursonn, ${ }^{35}$ D. Duchardt, ${ }^{35}$ M. Erdmann, ${ }^{35}$ R. Fischer, ${ }^{35}$ A. Güth, ${ }^{35}$ T. Hebbeker, ${ }^{35}$ C. Heidemann, ${ }^{35}$ K. Hoepfner, ${ }^{35}$ D. Klingebiel,${ }^{35}$ S. Knutzen, ${ }^{35}$ P. Kreuzer, ${ }^{35}$ M. Merschmeyer, ${ }^{35}$ A. Meyer, ${ }^{35}$ P. Millet,${ }^{35}$ M. Olschewski, ${ }^{35}$ K. Padeken, ${ }^{35}$ P. Papacz, ${ }^{35}$ H. Reithler, ${ }^{35}$ S. A. Schmitz, ${ }^{35}$ L. Sonnenschein, ${ }^{35}$ D. Teyssier, ${ }^{35}$ S. Thüer, ${ }^{35}$ M. Weber, ${ }^{35}$ V. Cherepanov, ${ }^{36}$ Y. Erdogan, ${ }^{36}$ G. Flügge ${ }^{36}$ H. Geenen, ${ }^{36}$ M. Geisler, ${ }^{36}$ W. Haj Ahmad, ${ }^{36}$ F. Hoehle, ${ }^{36}$ B. Kargoll,${ }^{36}$ T. Kress, ${ }^{36}$ Y. Kuessel, ${ }^{36}$ A. Künsken,${ }^{36}$ J. Lingemann, ${ }^{36, \mathrm{c}}$ A. Nowack, ${ }^{36}$ I. M. Nugent, ${ }^{36}$ L. Perchalla, ${ }^{36}$ O. Pooth, ${ }^{36}$ A. Stahl, ${ }^{36}$ M. Aldaya Martin, ${ }^{37}$ I. Asin, ${ }^{37}$ N. Bartosik, ${ }^{37}$ J. Behr, ${ }^{37}$ U. Behrens, ${ }^{37}$ A. J. Bell, ${ }^{37}$ A. Bethani, ${ }^{37}$ K. Borras, ${ }^{37}$ A. Burgmeier, ${ }^{37}$ A. Cakir, ${ }^{37}$ L. Calligaris, ${ }^{37}$ A. Campbell, ${ }^{37}$ S. Choudhury, ${ }^{37}$ F. Costanza,${ }^{37}$ C. Diez Pardos,${ }^{37}$ G. Dolinska, ${ }^{37}$ S. Dooling,${ }^{37}$ T. Dorland, ${ }^{37}$ G. Eckerlin,,${ }^{37}$ D. Eckstein, ${ }^{37}$ T. Eichhorn, ${ }^{37}$ G. Flucke, ${ }^{37}$ J. Garay Garcia, ${ }^{37}$ A. Geiser, ${ }^{37}$ P. Gunnellini, ${ }^{37}$ J. Hauk, ${ }^{37}$ M. Hempel, ${ }^{37, p}$ H. Jung, ${ }^{37}$ A. Kalogeropoulos,${ }^{37}$ M. Kasemann, ${ }^{37}$ P. Katsas, ${ }^{37}$ J. Kieseler, ${ }^{37}$ C. Kleinwort, ${ }^{37}$ I. Korol, ${ }^{37}$ D. Krücker, ${ }^{37}$

W. Lange, ${ }^{37}$ J. Leonard, ${ }^{37}$ K. Lipka, ${ }^{37}$ A. Lobanov, ${ }^{37}$ W. Lohmann, ${ }^{37, p}$ B. Lutz, ${ }^{37}$ R. Mankel, ${ }^{37}$ I. Marfin, ${ }^{37, p}$ I.-A. Melzer-Pellmann, ${ }^{37}$ A. B. Meyer, ${ }^{37}$ G. Mittag, ${ }^{37}$ J. Mnich, ${ }^{37}$ A. Mussgiller, ${ }^{37}$ S. Naumann-Emme, ${ }^{37}$ A. Nayak, ${ }^{37}$ E. Ntomari,${ }^{37}$ H. Perrey, ${ }^{37}$ D. Pitzl,${ }^{37}$ R. Placakyte, ${ }^{37}$ A. Raspereza, ${ }^{37}$ P. M. Ribeiro Cipriano, ${ }^{37}$ B. Roland, ${ }^{37}$ E. Ron, ${ }^{37}$ M. Ö. Sahin, ${ }^{37}$ J. Salfeld-Nebgen, ${ }^{37}$ P. Saxena, ${ }^{37}$ T. Schoerner-Sadenius, ${ }^{37}$ M. Schröder,${ }^{37}$ C. Seitz, ${ }^{37}$ S. Spannagel, ${ }^{37}$ A. D. R. Vargas Trevino, ${ }^{37}$ R. Walsh, ${ }^{37}$ C. Wissing, ${ }^{37}$ V. Blobel, ${ }^{38}$ M. Centis Vignali, ${ }^{38}$ A.r. Draeger, ${ }^{38}$ J. Erfle, ${ }^{38}$ E. Garutti, ${ }^{38}$ K. Goebel, ${ }^{38}$ M. Görner, ${ }^{38}$ J. Haller, ${ }^{38}$ M. Hoffmann, ${ }^{38}$ R. S. Höing, ${ }^{38}$ A. Junkes,${ }^{38}$ H. Kirschenmann, ${ }^{38}$ R. Klanner, ${ }^{38}$ R. Kogler, ${ }^{38}$ J. Lange, ${ }^{38}$ T. Lapsien, ${ }^{38}$ T. Lenz, ${ }^{38}$ I. Marchesini, ${ }^{38}$ J. Ott, ${ }^{38}$ T. Peiffer, ${ }^{38}$ A. Perieanu, ${ }^{38}$ N. Pietsch, ${ }^{38}$ J. Poehlsen, ${ }^{38}$ T. Poehlsen, ${ }^{38}$ D. Rathjens, ${ }^{38}$ C. Sander ${ }^{38}$ H. Schettler, ${ }^{38}$ P. Schleper, ${ }^{38}$ E. Schlieckau, ${ }^{38}$ A. Schmidt,${ }^{38}$ M. Seidel,${ }^{38}$ V. Sola,${ }^{38}$ H. Stadie,${ }^{38}$ G. Steinbrück, ${ }^{38}$ D. Troendle, ${ }^{38}$ E. Usai, ${ }^{38}$ L. Vanelderen, ${ }^{38}$ A. Vanhoefer,${ }^{38}$ C. Barth, ${ }^{39}$ C. Baus, ${ }^{39}$ J. Berger, ${ }^{39}$ C. Böser, ${ }^{39}$ E. Butz,${ }^{39}$ T. Chwalek, ${ }^{39}$ W. De Boer ${ }^{39}$ A. Descroix,${ }^{39}$ A. Dierlamm, ${ }^{39}$ M. Feindt, ${ }^{39}$ F. Frensch, ${ }^{39}$ M. Giffels, ${ }^{39}$ A. Gilbert, ${ }^{39}$ F. Hartmann,,${ }^{39, c}$ T. Hauth, ${ }^{39}$ U. Husemann, ${ }^{39}$ I. Katkov, ${ }^{39, f}$ A. Kornmayer, ${ }^{39, c}$ E. Kuznetsova ${ }^{39}$ P. Lobelle Pardo,${ }^{39}$ M. U. Mozer,${ }^{39}$ T. Müller, ${ }^{39}$ Th. Müller, ${ }^{39}$ A. Nürnberg, ${ }^{39}$ G. Quast,${ }^{39}$ K. Rabbertz, ${ }^{39}$ S. Röcker, ${ }^{39}$ H. J. Simonis, ${ }^{39}$ F. M. Stober, ${ }^{39}$ R. Ulrich, ${ }^{39}$ J. Wagner-Kuhr, ${ }^{39}$ S. Wayand, ${ }^{39}$ T. Weiler, ${ }^{39}$ R. Wolf, ${ }^{39}$ G. Anagnostou, ${ }^{40}$ G. Daskalakis, ${ }^{40}$ T. Geralis, ${ }^{40}$ V. A. Giakoumopoulou, ${ }^{40}$ A. Kyriakis, ${ }^{40}$ D. Loukas, ${ }^{40}$ A. Markou, ${ }^{40}$ C. Markou, ${ }^{40}$ A. Psallidas, ${ }^{40}$ I. Topsis-Giotis ${ }^{40}$ A. Agapitos, ${ }^{41}$ S. Kesisoglou, ${ }^{41}$ A. Panagiotou, ${ }^{41}$ N. Saoulidou, ${ }^{41}$ E. Stiliaris, ${ }^{41}$ X. Aslanoglou, ${ }^{42}$ I. Evangelou, ${ }^{42}$ G. Flouris,${ }^{42}$ C. Foudas, ${ }^{42}$ P. Kokkas, ${ }^{42}$ N. Manthos, ${ }^{42}$ I. Papadopoulos, ${ }^{42}$ E. Paradas,${ }^{42}$ J. Strologas, ${ }^{42}$ G. Bencze, ${ }^{43}$ C. Hajdu, ${ }^{43}$ P. Hidas, ${ }^{43}$ D. Horvath, ${ }^{43, q}$ F. Sikler, ${ }^{43}$ V. Veszpremi, ${ }^{43}$ G. Vesztergombi, ${ }^{43, r}$ A. J. Zsigmond, ${ }^{43}$ N. Beni, ${ }^{44}$ S. Czellar, ${ }^{44}$ J. Karancsii, ${ }^{44, s}$ J. Molnar, ${ }^{44}$ J. Palinkas, ${ }^{44}$ Z. Szillasi, ${ }^{44}$ A. Makovec ${ }^{45}$ P. Raics, ${ }^{45}$ Z. L. Trocsanyi, ${ }^{45}$ B. Ujvari, ${ }^{45}$ S. K. Swain, ${ }^{46}$ S. B. Beri, ${ }^{47}$ V. Bhatnagar, ${ }^{47}$ R. Gupta, ${ }^{47}$ U. Bhawandeep, ${ }^{47}$ A. K. Kalsi,${ }^{47}$ M. Kaur, ${ }^{47}$ R. Kumar, ${ }^{47}$ M. Mittal, ${ }^{47}$ N. Nishu, ${ }^{47}$ J. B. Singh,${ }^{47}$ Ashok Kumar, ${ }^{48}$ Arun Kumar, ${ }^{48}$ S. Ahuja, ${ }^{48}$ A. Bhardwaj, ${ }^{48}$ B. C. Choudhary, ${ }^{48}$ A. Kumar, ${ }^{48}$ S. Malhotra, ${ }^{48}$ M. Naimuddin, ${ }^{48}$ K. Ranjan, ${ }^{48}$ V. Sharma, ${ }^{48}$ S. Banerjee, ${ }^{49}$ S. Bhattacharya ${ }^{49}$ K. Chatterjee, ${ }^{49}$ S. Dutta, ${ }^{49}$ B. Gomber ${ }^{49}$ Sa. Jain, ${ }^{49}$ Sh. Jain, ${ }^{49}$ R. Khurana,${ }^{49}$ A. Modak, ${ }^{49}$ S. Mukherjee, ${ }^{49}$ D. Roy, ${ }^{49}$ S. Sarkar, ${ }^{49}$ M. Sharan, ${ }^{49}$ A. Abdulsalam, ${ }^{50}$ D. Dutta, ${ }^{50}$ S. Kailas,${ }^{50}$ V. Kumar,${ }^{50}$ A. K. Mohanty, ${ }^{50, \mathrm{c}}$ L. M. Pant,${ }^{50}$ P. Shukla ${ }^{50}$ A. Topkar, ${ }^{50}$ T. Aziz, ${ }^{51}$ S. Banerjee,${ }^{51}$ S. Bhowmik,${ }^{51, t}$ R. M. Chatterjee, ${ }^{51}$ R. K. Dewanjee, ${ }^{51}$ S. Dugad, ${ }^{51}$ S. Ganguly, ${ }^{51}$ S. Ghosh, ${ }^{51}$ M. Guchait, ${ }^{51}$ A. Gurtu, ${ }^{51, u}$ G. Kole, ${ }^{51}$ S. Kumar, ${ }^{51}$ M. Maity, ${ }^{51, t}$ G. Majumder, ${ }^{51}$ K. Mazumdar, ${ }^{51}$ G. B. Mohanty, ${ }^{51}$ B. Parida, ${ }^{51}$ K. Sudhakar, ${ }^{51}$ N. Wickramage, ${ }^{51, v}$ H. Bakhshiansohi, ${ }^{52}$ H. Behnamian, ${ }^{52}$ S. M. Etesami, ${ }^{52, w}$ A. Fahim, ${ }^{52, x}$ R. Goldouzian, ${ }^{52}$ M. Khakzad, ${ }^{52}$ M. Mohammadi Najafabadi, ${ }^{52}$ M. Naseri, ${ }^{52}$ S. Paktinat Mehdiabadi ${ }^{52}$ F. Rezaei Hosseinabadi, ${ }^{52}$ B. Safarzadeh, ${ }^{52, y}$ M. Zeinali, ${ }^{52}$ M. Felcini, ${ }^{53}$ M. Grunewald, ${ }^{53}$ M. Abbrescia, ${ }^{54,54 b}$ C. Calabria, ${ }^{54 a, 54 b}$ S. S. Chhibra, ${ }^{54 a, 54 b}$ A. Colaleo, ${ }^{54 a}$ D. Creanza, ${ }^{54 a, 54 c}$ N. De Filippis ${ }^{54 a, 54 c}$ M. De Palma, ${ }^{54 a, 54 b}$ L. Fiore,${ }^{54 a}$ G. Iaselli, ${ }^{54 a, 54 c}$ G. Maggi,${ }^{54,54 c}$ M. Maggi, ${ }^{54 a}$ S. My, ${ }^{54 a, 54 c}$ S. Nuzzo, ${ }^{54 a, 54 b}$ A. Pompili, ${ }^{54 a, 54 b}$ G. Pugliese,${ }^{54,54 c}$ R. Radogna, ${ }^{54 a, 54 b, c}$ G. Selvaggi, ${ }^{54,54 b}$ A. Sharma, ${ }^{54 a}$ L. Silvestris, ${ }^{54, c}$ R. Venditti, ${ }^{54 a, 54 b}$ P. Verwilligen, ${ }^{54 a}$ G. Abbiendi, ${ }^{55 a}$ A. C. Benvenuti, ${ }^{55 a}$ D. Bonacorsi, ${ }^{55 a, 55 b}$ S. Braibant-Giacomelli, ${ }^{55 a, 55 b}$ L. Brigliadori, ${ }^{55 a, 55 b}$ R. Campanini, ${ }^{55 a, 55 b}$ P. Capiluppi,${ }^{55 a, 55 b}$ A. Castro, ${ }^{55 a, 55 b}$ F. R. Cavallo, ${ }^{55 a}$ G. Codispoti, ${ }^{55 a, 55 b}$ 
M. Cuffiani, ${ }^{55,55 b}$ G. M. Dallavalle, ${ }^{55 a}$ F. Fabbri, ${ }^{55 a}$ A. Fanfani, ${ }^{55 a, 55 b}$ D. Fasanella ${ }^{55 a, 55 b}$ P. Giacomelli, ${ }^{55 a}$ C. Grandi, ${ }^{55 a}$ L. Guiducci, ${ }^{55 a, 55 b}$ S. Marcellini, ${ }^{55 a}$ G. Masetti, ${ }^{55 a}$ A. Montanari ${ }^{55 a}$ F. L. Navarria, ${ }^{55 a, 55 b}$ A. Perrotta, ${ }^{55 a}$ F. Primavera, ${ }^{55 a, 55 b}$ A. M. Rossi, ${ }^{55 a, 55 b}$ T. Rovelli, ${ }^{55 a, 55 b}$ G. P. Siroli,${ }^{55,55 b}$ N. Tosi, ${ }^{55,55 b}$ R. Travaglini, ${ }^{55 a, 55 b}$ S. Albergo, ${ }^{56 a, 56 b}$ G. Cappello, ${ }^{56 a}$ M. Chiorboli, ${ }^{56 a, 56 b}$ S. Costa ${ }^{56 a, 56 b}$ F. Giordano, ${ }^{56 a, 56 c, c}$ R. Potenza, ${ }^{56 a, 56 b}$ A. Tricomi, ${ }^{56 a, 56 b}$ C. Tuve, ${ }^{56 a, 56 b}$ G. Barbagli, ${ }^{57 a}$ V. Ciulli, ${ }^{57 a, 57 b}$ C. Civinini, ${ }^{57 a}$ R. D’Alessandro, ${ }^{57,57 b}$ E. Focardi, ${ }^{57,57 b}$ E. Gallo, ${ }^{57 a}$ S. Gonzi, ${ }^{57 a, 57 b}$ V. Gori, ${ }^{57 a, 57 b}$ P. Lenzi, ${ }^{57 a, 57 b}$ M. Meschini, ${ }^{57 a}$ S. Paoletti, ${ }^{57 a}$ G. Sguazzoni, ${ }^{57 a}$ A. Tropiano, ${ }^{57 a, 57 b}$ L. Benussi, ${ }^{58}$ S. Bianco, ${ }^{58}$ F. Fabbri, ${ }^{58}$

D. Piccolo, ${ }^{58}$ R. Ferretti ${ }^{59 a, 59 b}$ F. Ferro, ${ }^{59 a}$ M. Lo Vetere,${ }^{5 a, 59 b}$ E. Robutti, ${ }^{59 a}$ S. Tosi, ${ }^{59 a, 59 b}$ M. E. Dinardo, ${ }^{60 a, 60 b}$ S. Fiorendi, ${ }^{60 a, 60 b}$ S. Gennai, ${ }^{60 a, c}$ R. Gerosa,${ }^{60 a, 60 b, c}$ A. Ghezzi, ${ }^{60 a, 60 b}$ P. Govoni, ${ }^{60 a, 60 b}$ M. T. Lucchini, ${ }^{60 a, 60 b, c}$ S. Malvezzi, ${ }^{60 a}$ R. A. Manzoni,${ }^{60 a, 60 b}$ A. Martelli, ${ }^{60 a, 60 b}$ B. Marzocchi, ${ }^{60 a, 60 b, c}$ D. Menasce,${ }^{60 a}$ L. Moroni, ${ }^{60 a}$ M. Paganoni, ${ }^{60 a, 60 b}$ D. Pedrini, ${ }^{60 a}$ S. Ragazzi ${ }^{60 a, 60 b}$ N. Redaelli, ${ }^{60 \mathrm{a}}$ T. Tabarelli de Fatis,${ }^{60 \mathrm{a}, 60 \mathrm{~b}}$ S. Buontempo ${ }^{61 \mathrm{a}}$ N. Cavallo, ${ }^{61 \mathrm{a}, 61 \mathrm{c}} \mathrm{S}$. Di Guida, ${ }^{61 \mathrm{a}, 61 \mathrm{~d}, \mathrm{c}}$ F. Fabozzi, ${ }^{61,61 \mathrm{c}}$ A. O. M. Iorio, ${ }^{61 \mathrm{a}, 61 \mathrm{~b}}$ L. Lista, ${ }^{61 \mathrm{a}} \mathrm{S}$. Meola,${ }^{61 \mathrm{a}, 61 \mathrm{~d}, \mathrm{c}}$ M. Merola, ${ }^{61 \mathrm{a}}$ P. Paolucci, ${ }^{61 \mathrm{a}, \mathrm{c}} \mathrm{P}$. Azzi, ${ }^{62 \mathrm{a}} \mathrm{N}$. Bacchetta, ${ }^{62 \mathrm{a}}$ M. Biasotto ${ }^{62 a, z}$ D. Bisello, ${ }^{62 a, 62 b}$ A. Branca ${ }^{62 a, 62 b}$ R. Carlin, ${ }^{62 a, 62 b}$ P. Checchia ${ }^{62 a}$ M. Dall’Osso, ${ }^{62 a, 62 b}$ T. Dorigo, ${ }^{62 a}$ U. Dosselli, ${ }^{62 a}$ F. Fanzago, ${ }^{62 a}$ M. Galanti, ${ }^{62 a, 62 b}$ U. Gasparini, ${ }^{62 a, 62 b}$ P. Giubilato, ${ }^{62 a, 62 b}$ A. Gozzelino, ${ }^{62 a}$ S. Lacaprara, ${ }^{62 a}$ M. Margoni, ${ }^{62 a, 62 b}$ A. T. Meneguzzo, ${ }^{62 a, 62 b}$ J. Pazzini, ${ }^{62 a, 62 b}$ N. Pozzobon, ${ }^{62 a, 62 b}$ P. Ronchese, ${ }^{62 a, 62 b}$ F. Simonetto, ${ }^{62 a, 62 b}$ E. Torassa, ${ }^{62 a}$ M. Tosi, ${ }^{62 a, 62 b}$ S. Ventura,${ }^{62 a}$ P. Zotto,${ }^{62 a, 62 b}$ A. Zucchetta, ${ }^{62 a, 62 b}$ M. Gabusi, ${ }^{63 a, 63 b}$ S. P. Ratti, ${ }^{63 a, 63 b}$ V. Re,${ }^{63 a}$ C. Riccardi, ${ }^{63 a, 63 b}$ P. Salvini, ${ }^{63 a}$ P. Vitulo, ${ }^{63 a, 63 b}$ M. Biasini, ${ }^{64 a, 64 b}$ G. M. Bilei ${ }^{64 a}$ D. Ciangottini, ${ }^{64 a, 64 b, c}$ L. Fanò, ${ }^{64 a, 64 b}$ P. Lariccia,${ }^{64 a, 64 b}$ G. Mantovani ${ }^{64 a, 64 b}$ M. Menichelli, ${ }^{64 a}$ A. Saha ${ }^{64 a}$ A. Santocchia,${ }^{64 a, 64 b}$ A. Spiezia, ${ }^{64 a, 64 b, c}$ K. Androsov, ${ }^{65 a, a a}$ P. Azzurri, ${ }^{65 a}$ G. Bagliesi, ${ }^{65 a}$ J. Bernardini, ${ }^{65 a}$ T. Boccali, ${ }^{65 a}$ G. Broccolo $,{ }^{65 a}, 65 \mathrm{c}$ R. Castaldi, ${ }^{65 a}$ M. A. Ciocci, ${ }^{65 a, a a}$ R. Dell'Orso, ${ }^{65 a}$ S. Donato,${ }^{65 a, 65 c, c}$ F. Fiori, ${ }^{65 a, 65 c}$ L. Foà,${ }^{65 a, 65 c}$ A. Giassi, ${ }^{65 a}$ M. T. Grippo, ${ }^{65 a, a a}$ F. Ligabue ${ }^{65 a, 65 c}$ T. Lomtadze, ${ }^{65 a}$ L. Martini, ${ }^{65 a, 65 b}$ A. Messineo, ${ }^{65 a, 65 b}$ C. S. Moon, ${ }^{65 a, b b}$ F. Palla, ${ }^{65, c}$ A. Rizzi, ${ }^{65 a, 65 b}$ A. Savoy-Navarro, ${ }^{65 a, c c}$ A. T. Serban, ${ }^{65 a}$ P. Spagnolo, ${ }^{65 a}$ P. Squillacioti, ${ }^{65 a, a a}$ R. Tenchini, ${ }^{65 a}$ G. Tonelli, ${ }^{65 a, 65 b}$ A. Venturi, ${ }^{65 a}$ P. G. Verdini, ${ }^{65 \mathrm{a}}$ C. Vernieri, ${ }^{65 a, 65 \mathrm{c}}$ L. Barone ${ }^{66 \mathrm{a}, 66 \mathrm{~b}}$ F. Cavallari, ${ }^{66 \mathrm{a}}$ G. D’imperio, ${ }^{66 \mathrm{a}, 66 \mathrm{~b}}$ D. Del Re,${ }^{66 a, 66 \mathrm{~b}}$ M. Diemoz, ${ }^{66 \mathrm{a}}$ C. Jorda, ${ }^{66 \mathrm{a}}$ E. Longo, ${ }^{66 \mathrm{a}, 66 \mathrm{~b}}$ F. Margaroli, ${ }^{66 \mathrm{a}, 66 \mathrm{~b}}$ P. Meridiani, ${ }^{66 \mathrm{a}}$ F. Micheli, ${ }^{66 a, 66 \mathrm{~b}, \mathrm{c}}$ S. Nourbakhsh, ${ }^{66 \mathrm{a}, 66 \mathrm{~b}}$ G. Organtini, ${ }^{66 \mathrm{a}, 66 \mathrm{~b}}$ R. Paramatti, ${ }^{66 \mathrm{a}}$ S. Rahatlou, ${ }^{66 \mathrm{a}, 66 \mathrm{~b}}$ C. Rovelli, ${ }^{66 \mathrm{a}}$ F. Santanastasio, ${ }^{66 \mathrm{a}, 66 \mathrm{~b}}$ L. Soffi, ${ }^{66 a, 66 \mathrm{~b}}$ P. Traczyk, ${ }^{66 a, 66 b, c}$ N. Amapane, ${ }^{67 a, 67 b}$ R. Arcidiacono, ${ }^{67 \mathrm{a}, 67 \mathrm{c}}$ S. Argiro, ${ }^{67 a, 67 \mathrm{~b}}$ M. Arneodo, ${ }^{67 \mathrm{a}, 67 \mathrm{c}}$ R. Bellan, ${ }^{67 \mathrm{a}, 67 \mathrm{~b}}$ C. Biino, ${ }^{67 \mathrm{a}} \mathrm{N}$. Cartiglia, ${ }^{67 \mathrm{a}}$ S. Casasso, ${ }^{67 \mathrm{a}, 67 \mathrm{~b}, \mathrm{c}}$ M. Costa, ${ }^{67 a, 67 b}$ A. Degano, ${ }^{67 a, 67 b}$ N. Demaria, ${ }^{67 a}$ L. Finco, ${ }^{67 a, 67 b, c}$ C. Mariotti, ${ }^{67 a}$ S. Maselli, ${ }^{67 a}$ E. Migliore,${ }^{67 a, 67 b}$ V. Monaco, ${ }^{67 a, 67 b}$ M. Musich, ${ }^{67 a}$ M. M. Obertino, ${ }^{67 a, 67 c}$ G. Ortona, ${ }^{67 a, 67 b}$ L. Pacher, ${ }^{67 a, 67 b}$ N. Pastrone, ${ }^{67 a}$ M. Pelliccioni, ${ }^{67 a}$

G. L. Pinna Angioni, ${ }^{67 a, 67 b}$ A. Potenza, ${ }^{67 a, 67 b}$ A. Romero, ${ }^{67 a, 67 b}$ M. Ruspa ${ }^{67 a, 67 c}$ R. Sacchi, ${ }^{67 a, 67 b}$ A. Solano, ${ }^{67 a, 67 b}$ A. Staiano, ${ }^{67 \mathrm{a}}$ U. Tamponi, ${ }^{67 \mathrm{a}}$ S. Belforte, ${ }^{68 \mathrm{a}}$ V. Candelise,${ }^{68 \mathrm{a}, 68 \mathrm{~b}, \mathrm{c}}$ M. Casarsa, ${ }^{68 \mathrm{a}}$ F. Cossutti, ${ }^{68 \mathrm{a}}$ G. Della Ricca, ${ }^{68 \mathrm{a}, 68 \mathrm{~b}}$ B. Gobbo, ${ }^{68 \mathrm{a}}$ C. La Licata, ${ }^{68 \mathrm{a}, 68 \mathrm{~b}}$ M. Marone, ${ }^{68 \mathrm{a}, 68 \mathrm{~b}}$ A. Schizzi, ${ }^{68 \mathrm{a}, 68 \mathrm{~b}}$ T. Umer, ${ }^{68 \mathrm{a}, 68 \mathrm{~b}}$ A. Zanetti, ${ }^{68 \mathrm{a}} \mathrm{S}$. Chang, ${ }^{69}$ A. Kropivnitskaya, ${ }^{69}$ S. K. Nam, ${ }^{69}$ D. H. Kim, ${ }^{70}$ G. N. Kim, ${ }^{70}$ M. S. Kim, ${ }^{70}$ D. J. Kong, ${ }^{70}$ S. Lee, ${ }^{70}$ Y. D. Oh ${ }^{70}$ H. Park, ${ }^{70}$ A. Sakharov, ${ }^{70}$ D. C. Son, ${ }^{70}$ T. J. Kim, ${ }^{71}$ J. Y. Kim, ${ }^{72}$ S. Song, ${ }^{72}$ S. Choi, ${ }^{73}$ D. Gyun, ${ }^{73}$ B. Hong,${ }^{73}$ M. Jo, ${ }^{73}$ H. Kim, ${ }^{73}$ Y. Kim, ${ }^{73}$ B. Lee, ${ }^{73}$ K. S. Lee, ${ }^{73}$ S. K. Park ${ }^{73}$ Y. Roh, ${ }^{73}$ H. D. Yoo, ${ }^{74}$ M. Choi, ${ }^{75}$ J. H. Kim, ${ }^{75}$ I. C. Park,${ }^{75}$ G. Ryu ${ }^{75}$ M. S. Ryu, ${ }^{75}$ Y. Choi, ${ }^{76}$ Y. K. Choi,${ }^{76}$ J. Goh,${ }^{76}$ D. Kim, ${ }^{76}$ E. Kwon, ${ }^{76}$ J. Lee, ${ }^{76}$ I. Yu, ${ }^{76}$ A. Juodagalvis, ${ }^{77}$ J. R. Komaragiri, ${ }^{78}$ M. A. B. Md Ali, ${ }^{78}$ E. Casimiro Linares, ${ }^{79}$ H. Castilla-Valdez, ${ }^{79}$ E. De La Cruz-Burelo, ${ }^{79}$ I. Heredia-de La Cruz, ${ }^{79, d d}$ A. Hernandez-Almada, ${ }^{79}$ R. Lopez-Fernandez ${ }^{79}$ A. Sanchez-Hernandez ${ }^{79}$ S. Carrillo Moreno, ${ }^{80}$ F. Vazquez Valencia,${ }^{80}$ I. Pedraza, ${ }^{81}$ H. A. Salazar Ibarguen, ${ }^{81}$ A. Morelos Pineda, ${ }^{82}$ D. Krofcheck, ${ }^{83}$ P. H. Butler, ${ }^{84}$ S. Reucroft, ${ }^{84}$ A. Ahmad ${ }^{85}$ M. Ahmad, ${ }^{85}$ Q. Hassan ${ }^{85}$ H. R. Hoorani,${ }^{85}$ W. A. Khan,${ }^{85}$ T. Khurshid ${ }^{85}$ M. Shoaib, ${ }^{85}$ H. Bialkowska, ${ }^{86}$ M. Bluj ${ }^{86}$ B. Boimska, ${ }^{86}$ T. Frueboes, ${ }^{86}$ M. Górski, ${ }^{86}$ M. Kazana, ${ }^{86}$ K. Nawrocki ${ }^{86}$ K. Romanowska-Rybinska ${ }^{86}$ M. Szleper ${ }^{86}$ P. Zalewski, ${ }^{86}$ G. Brona,${ }^{87}$ K. Bunkowski, ${ }^{87}$ M. Cwiok ${ }^{87}$ W. Dominik ${ }^{87}$ K. Doroba, ${ }^{87}$ A. Kalinowski, ${ }^{87}$ M. Konecki ${ }^{87}$ J. Krolikowski, ${ }^{87}$ M. Misiura, ${ }^{87}$ M. Olszewski, ${ }^{87}$ W. Wolszczak, ${ }^{87}$ P. Bargassa, ${ }^{88}$ C. Beirão Da Cruz E Silva, ${ }^{88}$ P. Faccioli, ${ }^{88}$ P. G. Ferreira Parracho, ${ }^{88}$ M. Gallinaro, ${ }^{88}$ L. Lloret Iglesias, ${ }^{88}$ F. Nguyen, ${ }^{88}$ J. Rodrigues Antunes, ${ }^{88}$ J. Seixas,${ }^{88}$ J. Varela, ${ }^{88}$ P. Vischia, ${ }^{88}$ I. Golutvin, ${ }^{89}$ I. Gorbunov, ${ }^{89}$ A. Kamenev ${ }^{89}$ V. Karjavin, ${ }^{89}$ V. Konoplyanikov, ${ }^{89}$ G. Kozlov, ${ }^{89}$ A. Lanev, ${ }^{89}$ A. Malakhov, ${ }^{89}$ V. Matveev, ${ }^{89, e e}$ P. Moisenz,${ }^{89}$ V. Palichik, ${ }^{89}$ V. Perelygin, ${ }^{89}$ M. Savina, ${ }^{89}$ S. Shmatov ${ }^{89}$ S. Shulha, ${ }^{89}$ N. Skatchkov, ${ }^{89}$ V. Smirnov, ${ }^{89}$ A. Zarubin, ${ }^{89}$ V. Golovtsov, ${ }^{90}$ Y. Ivanov, ${ }^{90}$ V. Kim,,${ }^{90, f f}$ P. Levchenko, ${ }^{90}$ V. Murzin,${ }^{90}$ V. Oreshkin, ${ }^{90}$ I. Smirnov, ${ }^{90}$ V. Sulimov, ${ }^{90}$ L. Uvarov,${ }^{90}$ S. Vavilov, ${ }^{90}$ A. Vorobyev, ${ }^{90}$ An. Vorobyev,${ }^{90}$ Yu. Andreev, ${ }^{91}$ A. Dermenev, ${ }^{91}$ S. Gninenko, ${ }^{91}$ N. Golubev, ${ }^{91}$ M. Kirsanov, ${ }^{91}$ N. Krasnikov, ${ }^{91}$ A. Pashenkov, ${ }^{91}$ D. Tlisov, ${ }^{91}$ A. Toropin, ${ }^{91}$ V. Epshteyn,${ }^{92}$ V. Gavrilov, ${ }^{92}$ N. Lychkovskaya, ${ }^{92}$ V. Popov, ${ }^{92}$ I. Pozdnyakov, ${ }^{92}$ G. Safronov, ${ }^{92}$ S. Semenov, ${ }^{92}$ A. Spiridonov, ${ }^{92}$ V. Stolin, ${ }^{92}$ E. Vlasov, ${ }^{92}$ A. Zhokin, ${ }^{92}$ V. Andreev, ${ }^{93}$ M. Azarkin, ${ }^{93}$ I. Dremin, ${ }^{93}$ M. Kirakosyan, ${ }^{93}$ 
A. Leonidov, ${ }^{93}$ G. Mesyats, ${ }^{93}$ S. V. Rusakov, ${ }^{93}$ A. Vinogradov, ${ }^{93}$ A. Belyaev, ${ }^{94}$ E. Boos,${ }^{94}$ V. Bunichev,${ }^{94}$ M. Dubinin, ${ }^{94, g g}$ L. Dudko, ${ }^{94}$ A. Gribushin, ${ }^{94}$ V. Klyukhin, ${ }^{94}$ O. Kodolova,${ }^{94}$ I. Lokhtin,${ }^{94}$ S. Obraztsov, ${ }^{94}$ M. Perfilov, ${ }^{94}$ S. Petrushanko, ${ }^{94}$ V. Savrin, ${ }^{94}$ I. Azhgirey, ${ }^{95}$ I. Bayshev,${ }^{95}$ S. Bitioukov, ${ }^{95}$ V. Kachanov, ${ }^{95}$ A. Kalinin, ${ }^{95}$ D. Konstantinov,${ }^{95}$ V. Krychkine, ${ }^{95}$ V. Petrov ${ }^{95}$ R. Ryutin, ${ }^{95}$ A. Sobol, ${ }^{95}$ L. Tourtchanovitch, ${ }^{95}$ S. Troshin,${ }^{95}$ N. Tyurin, ${ }^{95}$ A. Uzunian, ${ }^{95}$ A. Volkov, ${ }^{95}$ P. Adzic, ${ }^{96, h h}$ M. Ekmedzic, ${ }^{96}$ J. Milosevic,${ }^{96}$ V. Rekovic,${ }^{96}$ J. Alcaraz Maestre, ${ }^{97}$ C. Battilana, ${ }^{97}$ E. Calvo, ${ }^{97}$ M. Cerrada, ${ }^{97}$ M. Chamizo Llatas, ${ }^{97}$ N. Colino, ${ }^{97}$ B. De La Cruz,${ }^{97}$ A. Delgado Peris, ${ }^{97}$ D. Domínguez Vázquez, ${ }^{97}$

A. Escalante Del Valle, ${ }^{97}$ C. Fernandez Bedoya, ${ }^{97}$ J. P. Fernández Ramos, ${ }^{97}$ J. Flix, ${ }^{97}$ M. C. Fouz, ${ }^{97}$ P. Garcia-Abia, ${ }^{97}$ O. Gonzalez Lopez, ${ }^{97}$ S. Goy Lopez, ${ }^{97}$ J. M. Hernandez,${ }^{97}$ M. I. Josa, ${ }^{97}$ E. Navarro De Martino, ${ }^{97}$

A. Pérez-Calero Yzquierdo, ${ }^{97}$ J. Puerta Pelayo, ${ }^{97}$ A. Quintario Olmeda, ${ }^{97}$ I. Redondo, ${ }^{97}$ L. Romero, ${ }^{97}$ M. S. Soares, ${ }^{97}$ C. Albajar, ${ }^{98}$ J. F. de Trocóniz, ${ }^{98}$ M. Missiroli, ${ }^{98}$ D. Moran, ${ }^{98}$ H. Brun, ${ }^{99}$ J. Cuevas, ${ }^{99}$ J. Fernandez Menendez,${ }^{99}$

S. Folgueras, ${ }^{99}$ I. Gonzalez Caballero, ${ }^{99}$ J. A. Brochero Cifuentes, ${ }^{100}$ I. J. Cabrillo, ${ }^{100}$ A. Calderon, ${ }^{100}$

J. Duarte Campderros, ${ }^{100}$ M. Fernandez, ${ }^{100}$ G. Gomez, ${ }^{100}$ A. Graziano, ${ }^{100}$ A. Lopez Virto, ${ }^{100}$ J. Marco,${ }^{100}$ R. Marco, ${ }^{100}$ C. Martinez Rivero, ${ }^{100}$ F. Matorras, ${ }^{100}$ F. J. Munoz Sanchez, ${ }^{100}$ J. Piedra Gomez, ${ }^{100}$ T. Rodrigo, ${ }^{100}$

A. Y. Rodríguez-Marrero, ${ }^{100}$ A. Ruiz-Jimeno, ${ }^{100}$ L. Scodellaro, ${ }^{100}$ I. Vila, ${ }^{100}$ R. Vilar Cortabitarte, ${ }^{100}$ D. Abbaneo, ${ }^{101}$ E. Auffray, ${ }^{101}$ G. Auzinger, ${ }^{101}$ M. Bachtis, ${ }^{101}$ P. Baillon, ${ }^{101}$ A. H. Ball, ${ }^{101}$ D. Barney, ${ }^{101}$ A. Benaglia, ${ }^{101}$ J. Bendavid, ${ }^{101}$ L. Benhabib, ${ }^{101}$ J. F. Benitez, ${ }^{101}$ C. Bernet, ${ }^{101, h}$ P. Bloch,${ }^{101}$ A. Bocci, ${ }^{101}$ A. Bonato, ${ }^{101}$ O. Bondu, ${ }^{101}$ C. Botta, ${ }^{101}$ H. Breuker, ${ }^{101}$ T. Camporesi, ${ }^{101}$ G. Cerminara, ${ }^{101}$ S. Colafranceschi, ${ }^{101, i i}$ M. D’Alfonso, ${ }^{101}$ D. d'Enterria, ${ }^{101}$ A. Dabrowski, ${ }^{101}$ A. David, ${ }^{101}$ F. De Guio, ${ }^{101}$ A. De Roeck, ${ }^{101}$ S. De Visscher, ${ }^{101}$ E. Di Marco, ${ }^{101}$ M. Dobson, ${ }^{101}$ M. Dordevic, ${ }^{101}$ B. Dorney, ${ }^{101}$ N. Dupont-Sagorin, ${ }^{101}$ A. Elliott-Peisert, ${ }^{101}$ J. Eugster, ${ }^{101}$ G. Franzoni, ${ }^{101}$ W. Funk, ${ }^{101}$ D. Gigi,${ }^{101}$ K. Gill, ${ }^{101}$ D. Giordano, ${ }^{101}$ M. Girone, ${ }^{101}$ F. Glege,${ }^{101}$ R. Guida,${ }^{101}$ S. Gundacker, ${ }^{101}$ M. Guthoff, ${ }^{101}$ J. Hammer, ${ }^{101}$ M. Hansen, ${ }^{101}$ P. Harris, ${ }^{101}$ J. Hegeman, ${ }^{101}$ V. Innocente,${ }^{101}$ P. Janot, ${ }^{101}$ K. Kousouris,${ }^{101}$ K. Krajczar, ${ }^{101}$ P. Lecoq, ${ }^{101}$ C. Lourenço, ${ }^{101}$ N. Magini, ${ }^{101}$ L. Malgeri, ${ }^{101}$ M. Mannelli, ${ }^{101}$ J. Marrouche, ${ }^{101}$ L. Masetti, ${ }^{101}$ F. Meijers, ${ }^{101}$ S. Mersi, ${ }^{101}$ E. Meschi,${ }^{101}$ F. Moortgat,${ }^{101}$ S. Morovic,${ }^{101}$ M. Mulders, ${ }^{101}$ L. Orsini, ${ }^{101}$ L. Pape,${ }^{101}$ E. Perez,${ }^{101}$ L. Perrozzi,${ }^{101}$ A. Petrilli, ${ }^{101}$ G. Petrucciani, ${ }^{101}$ A. Pfeiffer,${ }^{101}$ M. Pimiä, ${ }^{101}$ D. Piparo,${ }^{101}$ M. Plagge,${ }^{101}$ A. Racz, ${ }^{101}$ G. Rolandi, ${ }^{101, j j}$ M. Rovere, ${ }^{101}$ H. Sakulin, ${ }^{101}$ C. Schäfer, ${ }^{101}$ C. Schwick, ${ }^{101}$ A. Sharma, ${ }^{101}$ P. Siegrist, ${ }^{101}$ P. Silva, ${ }^{101}$ M. Simon, ${ }^{101}$ P. Sphicas ${ }^{101, \mathrm{kk}}$ D. Spiga, ${ }^{101}$ J. Steggemann, ${ }^{101}$ B. Stieger ${ }^{101}$ M. Stoye,${ }^{101}$ Y. Takahashi, ${ }^{101}$ D. Treille, ${ }^{101}$ A. Tsirou, ${ }^{101}$ G. I. Veres, ${ }^{101, \mathrm{r}}$ N. Wardle, ${ }^{101}$ H. K. Wöhri, ${ }^{101}$ H. Wollny, ${ }^{101}$ W. D. Zeuner, ${ }^{101}$ W. Bertl, ${ }^{102}$ K. Deiters, ${ }^{102}$ W. Erdmann, ${ }^{102}$ R. Horisberger, ${ }^{102}$ Q. Ingram, ${ }^{102}$ H. C. Kaestli, ${ }^{102}$ D. Kotlinski, ${ }^{102}$ U. Langenegger, ${ }^{102}$ D. Renker,${ }^{102}$ T. Rohe,${ }^{102}$ F. Bachmair, ${ }^{103}$ L. Bäni, ${ }^{103}$ L. Bianchini, ${ }^{103}$ M. A. Buchmann, ${ }^{103}$ B. Casal, ${ }^{103}$ N. Chanon, ${ }^{103}$ G. Dissertori, ${ }^{103}$ M. Dittmar, ${ }^{103}$ M. Donegà, ${ }^{103}$ M. Dünser, ${ }^{103}$ P. Eller, ${ }^{103}$ C. Grab,${ }^{103}$ D. Hits, ${ }^{103}$ J. Hoss, ${ }^{103}$ W. Lustermann,,${ }^{103}$ B. Mangano, ${ }^{103}$ A. C. Marini, ${ }^{103}$ M. Marionneau, ${ }^{103}$ P. Martinez Ruiz del Arbol, ${ }^{103}$ M. Masciovecchio, ${ }^{103}$ D. Meister, ${ }^{103}$ N. Mohr, ${ }^{103}$ P. Musella, ${ }^{103}$ C. Nägeli, ${ }^{103,11}$ F. Nessi-Tedaldi, ${ }^{103}$ F. Pandolfi, ${ }^{103}$ F. Pauss, ${ }^{103}$ M. Peruzzi, ${ }^{103}$ M. Quittnat,${ }^{103}$ L. Rebane, ${ }^{103}$ M. Rossini, ${ }^{103}$ A. Starodumov, ${ }^{103, \mathrm{~mm}}$ M. Takahashi, ${ }^{103}$ K. Theofilatos, ${ }^{103}$ R. Wallny ${ }^{103}$ H. A. Weber ${ }^{103}$ C. Amsler,${ }^{104, n n}$ M. F. Canelli, ${ }^{104}$ V. Chiochia,${ }^{104}$ A. De Cosa, ${ }^{104}$ A. Hinzmann, ${ }^{104}$ T. Hreus, ${ }^{104}$ B. Kilminster,${ }^{104}$ C. Lange,${ }^{104}$ B. Millan Mejias, ${ }^{104}$ J. Ngadiuba, ${ }^{104}$ D. Pinna, ${ }^{104}$ P. Robmann, ${ }^{104}$ F. J. Ronga, ${ }^{104}$ S. Taroni, ${ }^{104}$ M. Verzetti, ${ }^{104}$ Y. Yang, ${ }^{104}$ M. Cardaci, ${ }^{105}$ K. H. Chen, ${ }^{105}$ C. Ferro, ${ }^{105}$ C. M. Kuo, ${ }^{105}$ W. Lin, ${ }^{105}$ Y. J. Lu, ${ }^{105}$ R. Volpe, ${ }^{105}$ S. S. Yu, ${ }^{105}$ P. Chang, ${ }^{106}$ Y. H. Chang, ${ }^{106}$ Y. W. Chang, ${ }^{106}$ Y. Chao, ${ }^{106}$ K. F. Chen, ${ }^{106}$ P. H. Chen, ${ }^{106}$ C. Dietz, ${ }^{106}$ U. Grundler, ${ }^{106}$ W.-S. Hou, ${ }^{106}$ K. Y. Kao, ${ }^{106}$ Y. F. Liu, ${ }^{106}$ R.-S. Lu ${ }^{106}$ D. Majumder, ${ }^{106}$ E. Petrakou, ${ }^{106}$ Y. M. Tzeng, ${ }^{106}$ R. Wilken, ${ }^{106}$ B. Asavapibhop, ${ }^{107}$ G. Singh, ${ }^{107}$ N. Srimanobhas, ${ }^{107}$ N. Suwonjandee, ${ }^{107}$ A. Adiguzel,${ }^{108}$ M. N. Bakirci, ${ }^{108,00}$ S. Cerci, ${ }^{108, p p}$ C. Dozen,${ }^{108}$ I. Dumanoglu, ${ }^{108}$ E. Eskut, ${ }^{108}$ S. Girgis, ${ }^{108}$ G. Gokbulut, ${ }^{108}$ E. Gurpinar, ${ }^{108}$ I. Hos, ${ }^{108}$ E. E. Kangal, ${ }^{108}$ A. Kayis Topaksu, ${ }^{108}$ G. Onengut, ${ }^{108, q q}$ K. Ozdemir, ${ }^{108}$ S. Ozturk, ${ }^{108, \text { oo }}$ A. Polatoz, ${ }^{108}$ D. Sunar Cerci, ${ }^{108, p p}$ B. Tali, ${ }^{108, p p}$ H. Topakli, ${ }^{108,00}$ M. Vergili, ${ }^{108}$ I. V. Akin, ${ }^{109}$ B. Bilin, ${ }^{109}$ S. Bilmis, ${ }^{109}$ H. Gamsizkan, ${ }^{109, \text { rr }}$ B. Isildak, ${ }^{109, s s}$ G. Karapinar, ${ }^{109, t t}$ K. Ocalan, ${ }^{109, \text { uu }}$ S. Sekmen, ${ }^{109}$ U. E. Surat, ${ }^{109}$ M. Yalvac,${ }^{109}$ M. Zeyrek, ${ }^{109}$ E. A. Albayrak,,${ }^{110, v v}$ E. Gülmez,${ }^{110}$ M. Kaya, ${ }^{110, w w}$ O. Kaya, ${ }^{110, x x}$ T. Yetkin, ${ }^{110, y y}$ K. Cankocak, ${ }^{111}$ F. I. Vardarl1, ${ }^{111}$ L. Levchuk, ${ }^{112}$ P. Sorokin, ${ }^{112}$ J. J. Brooke, ${ }^{113}$ E. Clement, ${ }^{113}$ D. Cussans, ${ }^{113}$ H. Flacher, ${ }^{113}$ J. Goldstein, ${ }^{113}$ M. Grimes, ${ }^{113}$ G. P. Heath, ${ }^{113}$ H. F. Heath, ${ }^{113}$ J. Jacob,${ }^{113}$ L. Kreczko, ${ }^{113}$ C. Lucas, ${ }^{113}$ Z. Meng, ${ }^{113}$ D. M. Newbold, ${ }^{113, z z}$ S. Paramesvaran, ${ }^{113}$ A. Poll, ${ }^{113}$ T. Sakuma, ${ }^{113}$ S. Senkin, ${ }^{113}$ V. J. Smith, ${ }^{113}$ T. Williams, ${ }^{113}$

K. W. Bell, ${ }^{114}$ A. Belyaev, ${ }^{114, \text { aaa }}$ C. Brew, ${ }^{114}$ R. M. Brown, ${ }^{114}$ D. J. A. Cockerill, ${ }^{114}$ J. A. Coughlan, ${ }^{114}$ K. Harder, ${ }^{114}$ S. Harper, ${ }^{114}$ E. Olaiya, ${ }^{114}$ D. Petyt, ${ }^{114}$ C. H. Shepherd-Themistocleous, ${ }^{114}$ A. Thea, ${ }^{114}$ I. R. Tomalin, ${ }^{114}$ W. J. Womersley, ${ }^{114}$ S. D. Worm, ${ }^{114}$ M. Baber, ${ }^{115}$ R. Bainbridge, ${ }^{115}$ O. Buchmuller, ${ }^{115}$ D. Burton, ${ }^{115}$ D. Colling, ${ }^{115}$ N. Cripps, ${ }^{115}$ P. Dauncey, ${ }^{115}$ 
G. Davies, ${ }^{115}$ M. Della Negra, ${ }^{115}$ P. Dunne, ${ }^{115}$ W. Ferguson, ${ }^{115}$ J. Fulcher, ${ }^{115}$ D. Futyan, ${ }^{115}$ G. Hall, ${ }^{115}$ G. Iles, ${ }^{115}$ M. Jarvis, ${ }^{115}$ G. Karapostoli, ${ }^{115}$ M. Kenzie, ${ }^{115}$ R. Lane, ${ }^{115}$ R. Lucas, ${ }^{115, z z}$ L. Lyons, ${ }^{115}$ A.-M. Magnan, ${ }^{115}$ S. Malik, ${ }^{115}$ B. Mathias, ${ }^{115}$ J. Nash, ${ }^{115}$ A. Nikitenko, ${ }^{115, \mathrm{~mm}}$ J. Pela, ${ }^{115}$ M. Pesaresi, ${ }^{115}$ K. Petridis, ${ }^{115}$ D. M. Raymond ${ }^{115}$ S. Rogerson, ${ }^{115}$ A. Rose, ${ }^{115}$ C. Seez, ${ }^{115}$ P. Sharp, ${ }^{115, a}$ A. Tapper, ${ }^{115}$ M. Vazquez Acosta, ${ }^{115}$ T. Virdee, ${ }^{115}$ S. C. Zenz, ${ }^{115}$ J. E. Cole, ${ }^{116}$ P. R. Hobson, ${ }^{116}$ A. Khan, ${ }^{116}$ P. Kyberd, ${ }^{116}$ D. Leggat,${ }^{116}$ D. Leslie, ${ }^{116}$ I. D. Reid, ${ }^{116}$ P. Symonds, ${ }^{116}$ L. Teodorescu, ${ }^{116}$ M. Turner, ${ }^{116}$

J. Dittmann, ${ }^{117}$ K. Hatakeyama, ${ }^{117}$ A. Kasmi ${ }^{117}$ H. Liu, ${ }^{117}$ T. Scarborough, ${ }^{117}$ O. Charaf, ${ }^{118}$ S. I. Cooper, ${ }^{118}$ C. Henderson, ${ }^{118}$ P. Rumerio, ${ }^{118}$ A. Avetisyan, ${ }^{119}$ T. Bose, ${ }^{119}$ C. Fantasia, ${ }^{119}$ P. Lawson, ${ }^{119}$ C. Richardson, ${ }^{119}$ J. Rohlf, ${ }^{119}$ J. St. John, ${ }^{119}$ L. Sulak, ${ }^{119}$ J. Alimena, ${ }^{120}$ E. Berry, ${ }^{120}$ S. Bhattacharya, ${ }^{120}$ G. Christopher, ${ }^{120}$ D. Cutts, ${ }^{120}$ Z. Demiragli, ${ }^{120}$ N. Dhingra, ${ }^{120}$ A. Ferapontov, ${ }^{120}$ A. Garabedian, ${ }^{120}$ U. Heintz, ${ }^{120}$ G. Kukartsev,${ }^{120}$ E. Laird, ${ }^{120}$ G. Landsberg, ${ }^{120}$ M. Luk, ${ }^{120}$ M. Narain, ${ }^{120}$ M. Segala, ${ }^{120}$ T. Sinthuprasith, ${ }^{120}$ T. Speer, ${ }^{120}$ J. Swanson, ${ }^{120}$ R. Breedon, ${ }^{121}$ G. Breto, ${ }^{121}$

M. Calderon De La Barca Sanchez, ${ }^{121}$ S. Chauhan, ${ }^{121}$ M. Chertok, ${ }^{121}$ J. Conway, ${ }^{121}$ R. Conway, ${ }^{121}$ P. T. Cox ${ }^{121}$ R. Erbacher, ${ }^{121}$ M. Gardner, ${ }^{121}$ W. Ko, ${ }^{121}$ R. Lander, ${ }^{121}$ T. Miceli, ${ }^{121}$ M. Mulhearn, ${ }^{121}$ D. Pellett, ${ }^{121}$ J. Pilot, ${ }^{121}$

F. Ricci-Tam, ${ }^{121}$ M. Searle, ${ }^{121}$ S. Shalhout, ${ }^{121}$ J. Smith, ${ }^{121}$ M. Squires, ${ }^{121}$ D. Stolp, ${ }^{121}$ M. Tripathi, ${ }^{121}$ S. Wilbur, ${ }^{121}$ R. Yohay, ${ }^{121}$ R. Cousins,${ }^{122}$ P. Everaerts, ${ }^{122}$ C. Farrell, ${ }^{122}$ J. Hauser, ${ }^{122}$ M. Ignatenko, ${ }^{122}$ G. Rakness, ${ }^{122}$ E. Takasugi, ${ }^{122}$ V. Valuev, ${ }^{122}$ M. Weber, ${ }^{122}$ K. Burt, ${ }^{123}$ R. Clare,${ }^{123}$ J. Ellison, ${ }^{123}$ J. W. Gary, ${ }^{123}$ G. Hanson, ${ }^{123}$ J. Heilman, ${ }^{123}$ M. Ivova Rikova, ${ }^{123}$ P. Jandir, ${ }^{123}$ E. Kennedy, ${ }^{123}$ F. Lacroix, ${ }^{123}$ O. R. Long ${ }^{123}$ A. Luthra, ${ }^{123}$ M. Malberti, ${ }^{123}$ M. Olmedo Negrete, ${ }^{123}$ A. Shrinivas, ${ }^{123}$ S. Sumowidagdo, ${ }^{123}$ S. Wimpenny, ${ }^{123}$ J. G. Branson, ${ }^{124}$ G. B. Cerati, ${ }^{124}$ S. Cittolin, ${ }^{124}$ R. T. D’Agnolo, ${ }^{124}$ A. Holzner, ${ }^{124}$ R. Kelley, ${ }^{124}$ D. Klein, ${ }^{124}$ J. Letts, ${ }^{124}$ I. Macneill, ${ }^{124}$ D. Olivito, ${ }^{124}$ S. Padhi, ${ }^{124}$ C. Palmer, ${ }^{124}$ M. Pieri, ${ }^{124}$ M. Sani, ${ }^{124}$ V. Sharma, ${ }^{124}$ S. Simon, ${ }^{124}$ M. Tadel,${ }^{124}$ Y. Tu, ${ }^{124}$ A. Vartak, ${ }^{124}$ C. Welke, ${ }^{124}$ F. Würthwein, ${ }^{124}$ A. Yagil, ${ }^{124}$ D. Barge, ${ }^{125}$ J. Bradmiller-Feld, ${ }^{125}$ C. Campagnari, ${ }^{125}$ T. Danielson, ${ }^{125}$ A. Dishaw, ${ }^{125}$ V. Dutta, ${ }^{125}$ K. Flowers, ${ }^{125}$ M. Franco Sevilla, ${ }^{125}$ P. Geffert, ${ }^{125}$ C. George, ${ }^{125}$ F. Golf, ${ }^{125}$ L. Gouskos, ${ }^{125}$ J. Incandela, ${ }^{125}$ C. Justus, ${ }^{125}$ N. Mccoll,,${ }^{125}$ J. Richman, ${ }^{125}$ D. Stuart, ${ }^{125}$ W. To, ${ }^{125}$ C. West, ${ }^{125}$ J. Yoo, ${ }^{125}$ A. Apresyan, ${ }^{126}$ A. Bornheim, ${ }^{126}$ J. Bunn, ${ }^{126}$ Y. Chen, ${ }^{126}$ J. Duarte, ${ }^{126}$ A. Mott, ${ }^{126}$ H. B. Newman, ${ }^{126}$ C. Pena, ${ }^{126}$ M. Pierini, ${ }^{126}$ M. Spiropulu, ${ }^{126}$ J. R. Vlimant, ${ }^{126}$ R. Wilkinson, ${ }^{126}$ S. Xie, ${ }^{126}$ R. Y. Zhu, ${ }^{126}$ V. Azzolini, ${ }^{127}$ A. Calamba, ${ }^{127}$ B. Carlson, ${ }^{127}$ T. Ferguson, ${ }^{127}$ Y. Iiyama, ${ }^{127}$ M. Paulini, ${ }^{127}$ J. Russ, ${ }^{127}$ H. Vogel,${ }^{127}$ I. Vorobiev, ${ }^{127}$ J. P. Cumalat, ${ }^{128}$ W. T. Ford, ${ }^{128}$ A. Gaz, ${ }^{128}$ M. Krohn, ${ }^{128}$ E. Luiggi Lopez, ${ }^{128}$ U. Nauenberg, ${ }^{128}$ J. G. Smith, ${ }^{128}$ K. Stenson, ${ }^{128}$ K. A. Ulmer, ${ }^{128}$ S. R. Wagner, ${ }^{128}$ J. Alexander, ${ }^{129}$ A. Chatterjee, ${ }^{129}$ J. Chaves, ${ }^{129}$ J. Chu ${ }^{129}$ S. Dittmer, ${ }^{129}$ N. Eggert, ${ }^{129}$ N. Mirman, ${ }^{129}$ G. Nicolas Kaufman, ${ }^{129}$ J. R. Patterson, ${ }^{129}$ A. Ryd ${ }^{129}$ E. Salvati, ${ }^{129}$ L. Skinnari, ${ }^{129}$ W. Sun, ${ }^{129}$ W. D. Teo, ${ }^{129}$ J. Thom, ${ }^{129}$ J. Thompson, ${ }^{129}$ J. Tucker, ${ }^{129}$ Y. Weng, ${ }^{129}$ L. Winstrom, ${ }^{129}$ P. Wittich, ${ }^{129}$ D. Winn, ${ }^{130}$ S. Abdullin, ${ }^{131}$ M. Albrow ${ }^{131}$ J. Anderson, ${ }^{131}$ G. Apollinari, ${ }^{131}$

L. A. T. Bauerdick, ${ }^{131}$ A. Beretvas, ${ }^{131}$ J. Berryhill, ${ }^{131}$ P. C. Bhat, ${ }^{131}$ G. Bolla, ${ }^{131}$ K. Burkett, ${ }^{131}$ J. N. Butler, ${ }^{131}$

H. W. K. Cheung, ${ }^{131}$ F. Chlebana, ${ }^{131}$ S. Cihangir, ${ }^{131}$ V. D. Elvira, ${ }^{131}$ I. Fisk, ${ }^{131}$ J. Freeman, ${ }^{131}$ Y. Gao, ${ }^{131}$ E. Gottschalk, ${ }^{131}$ L. Gray, ${ }^{131}$ D. Green, ${ }^{131}$ S. Grünendahl, ${ }^{131}$ O. Gutsche, ${ }^{131}$ J. Hanlon, ${ }^{131}$ D. Hare, ${ }^{131}$ R. M. Harris, ${ }^{131}$ J. Hirschauer, ${ }^{131}$ B. Hooberman, ${ }^{131}$ S. Jindariani, ${ }^{131}$ M. Johnson, ${ }^{131}$ U. Joshi, ${ }^{131}$ K. Kaadze, ${ }^{131}$ B. Klima, ${ }^{131}$ B. Kreis, ${ }^{131}$ S. Kwan, ${ }^{131, a}$ J. Linacre, ${ }^{131}$ D. Lincoln, ${ }^{131}$ R. Lipton, ${ }^{131}$ T. Liu, ${ }^{131}$ J. Lykken, ${ }^{131}$ K. Maeshima, ${ }^{131}$ J. M. Marraffino, ${ }^{131}$ V. I. Martinez Outschoorn, ${ }^{131}$ S. Maruyama, ${ }^{131}$ D. Mason, ${ }^{131}$ P. McBride, ${ }^{131}$ P. Merkel, ${ }^{131}$ K. Mishra ${ }^{131}$ S. Mrenna, ${ }^{131}$ S. Nahn, ${ }^{131}$ C. Newman-Holmes, ${ }^{131}$ V. O’Dell, ${ }^{131}$ O. Prokofyev, ${ }^{131}$ E. Sexton-Kennedy, ${ }^{131}$ S. Sharma, ${ }^{131}$ A. Soha, ${ }^{131}$ W. J. Spalding, ${ }^{131}$ L. Spiegel, ${ }^{131}$ L. Taylor, ${ }^{131}$ S. Tkaczyk, ${ }^{131}$ N. V. Tran,${ }^{131}$ L. Uplegger, ${ }^{131}$ E. W. Vaandering,,${ }^{131}$ R. Vidal, ${ }^{131}$ A. Whitbeck, ${ }^{131}$ J. Whitmore, ${ }^{131}$ F. Yang, ${ }^{131}$ D. Acosta, ${ }^{132}$ P. Avery, ${ }^{132}$ P. Bortignon, ${ }^{132}$ D. Bourilkov, ${ }^{132}$ M. Carver, ${ }^{132}$ D. Curry, ${ }^{132}$ S. Das, ${ }^{132}$ M. De Gruttola, ${ }^{132}$ G. P. Di Giovanni, ${ }^{132}$ R. D. Field, ${ }^{132}$ M. Fisher, ${ }^{132}$ I. K. Furic, ${ }^{132}$ J. Hugon, ${ }^{132}$ J. Konigsberg, ${ }^{132}$ A. Korytov, ${ }^{132}$ T. Kypreos, ${ }^{132}$ J. F. Low, ${ }^{132}$ K. Matchev, ${ }^{132}$ H. Mei, ${ }^{132}$ P. Milenovic, ${ }^{132, b b b}$ G. Mitselmakher, ${ }^{132}$ L. Muniz, ${ }^{132}$ A. Rinkevicius, ${ }^{132}$ L. Shchutska, ${ }^{132}$ M. Snowball, ${ }^{132}$ D. Sperka, ${ }^{132}$ J. Yelton, ${ }^{132}$ M. Zakaria, ${ }^{132}$ S. Hewamanage, ${ }^{133}$ S. Linn, ${ }^{133}$ P. Markowitz, ${ }^{133}$ G. Martinez, ${ }^{133}$ J. L. Rodriguez, ${ }^{133}$ T. Adams, ${ }^{134}$ A. Askew, ${ }^{134}$ J. Bochenek, ${ }^{134}$ B. Diamond, ${ }^{134}$ J. Haas, ${ }^{134}$ S. Hagopian, ${ }^{134}$ V. Hagopian, ${ }^{134}$ K. F. Johnson, ${ }^{134}$ H. Prosper, ${ }^{134}$ V. Veeraraghavan, ${ }^{134}$ M. Weinberg, ${ }^{134}$ M. M. Baarmand ${ }^{135}$ M. Hohlmann,${ }^{135}$ H. Kalakhety, ${ }^{135}$ F. Yumiceva, ${ }^{135}$ M. R. Adams, ${ }^{136}$ L. Apanasevich, ${ }^{136}$ D. Berry, ${ }^{136}$ R. R. Betts, ${ }^{136}$ I. Bucinskaite, ${ }^{136}$ R. Cavanaugh, ${ }^{136}$ O. Evdokimov, ${ }^{136}$ L. Gauthier, ${ }^{136}$ C. E. Gerber, ${ }^{136}$ D. J. Hofman, ${ }^{136}$ P. Kurt, ${ }^{136}$ D. H. Moon, ${ }^{136}$ C. O’Brien, ${ }^{136}$ I. D. Sandoval Gonzalez, ${ }^{136}$ C. Silkworth, ${ }^{136}$ P. Turner, ${ }^{136}$ N. Varelas, ${ }^{136}$ B. Bilki, ${ }^{137, c c c}$ W. Clarida,${ }^{137}$ K. Dilsiz, ${ }^{137}$ M. Haytmyradov, ${ }^{137}$ J.-P. Merlo, ${ }^{137}$ H. Mermerkaya, ${ }^{137, \text { ddd }}$ A. Mestvirishvili, ${ }^{137}$ A. Moeller, ${ }^{137}$ J. Nachtman, ${ }^{137}$ H. Ogul, ${ }^{137}$ Y. Onel,,${ }^{137}$ F. Ozok, ${ }^{137, v v}$ A. Penzo, ${ }^{137}$ R. Rahmat, ${ }^{137}$ S. Sen, ${ }^{137}$ P. Tan, ${ }^{137}$ E. Tiras, ${ }^{137}$ J. Wetzel, ${ }^{137}$ K. Yi, ${ }^{137}$ B. A. Barnett, ${ }^{138}$ B. Blumenfeld, ${ }^{138}$ 
S. Bolognesi ${ }^{138}$ D. Fehling, ${ }^{138}$ A. V. Gritsan, ${ }^{138}$ P. Maksimovic, ${ }^{138}$ C. Martin, ${ }^{138}$ M. Swartz,${ }^{138}$ P. Baringer, ${ }^{139}$ A. Bean, ${ }^{139}$ G. Benelli, ${ }^{139}$ C. Bruner, ${ }^{139}$ R. P. Kenny III, ${ }^{139}$ M. Malek, ${ }^{139}$ M. Murray, ${ }^{139}$ D. Noonan, ${ }^{139}$ S. Sanders, ${ }^{139}$ J. Sekaric,,${ }^{139}$ R. Stringer, ${ }^{139}$ Q. Wang, ${ }^{139}$ J. S. Wood ${ }^{139}$ I. Chakaberia, ${ }^{140}$ A. Ivanov, ${ }^{140}$ S. Khalil, ${ }^{140}$ M. Makouski, ${ }^{140}$ Y. Maravin, ${ }^{140}$ L. K. Saini, ${ }^{140}$ N. Skhirtladze, ${ }^{140}$ I. Svintradze, ${ }^{140}$ J. Gronberg, ${ }^{141}$ D. Lange,${ }^{141}$ F. Rebassoo, ${ }^{141}$ D. Wright,${ }^{141}$ A. Baden, ${ }^{142}$ A. Belloni, ${ }^{142}$ B. Calvert, ${ }^{142}$ S. C. Eno, ${ }^{142}$ J. A. Gomez, ${ }^{142}$ N. J. Hadley, ${ }^{142}$ R. G. Kellogg, ${ }^{142}$ T. Kolberg, ${ }^{142}$ Y. Lu, ${ }^{142}$ A. C. Mignerey, ${ }^{142}$ K. Pedro, ${ }^{142}$ A. Skuja, ${ }^{142}$ M. B. Tonjes, ${ }^{142}$ S. C. Tonwar, ${ }^{142}$ A. Apyan,${ }^{143}$ R. Barbieri, ${ }^{143}$ G. Bauer, ${ }^{143}$ W. Busza, ${ }^{143}$ I. A. Cali, ${ }^{143}$ M. Chan, ${ }^{143}$ L. Di Matteo, ${ }^{143}$ G. Gomez Ceballos,${ }^{143}$ M. Goncharov,${ }^{143}$ D. Gulhan ${ }^{143}$ M. Klute, ${ }^{143}$ Y. S. Lai, ${ }^{143}$ Y.-J. Lee, ${ }^{143}$ A. Levin, ${ }^{143}$ P. D. Luckey, ${ }^{143}$ T. Ma,${ }^{143}$ C. Paus,${ }^{143}$ D. Ralph,${ }^{143}$ C. Roland, ${ }^{143}$ G. Roland, ${ }^{143}$ G. S. F. Stephans, ${ }^{143}$ F. Stöckli, ${ }^{143}$ K. Sumorok, ${ }^{143}$ D. Velicanu, ${ }^{143}$ J. Veverka, ${ }^{143}$ B. Wyslouch,${ }^{143}$ M. Yang, ${ }^{143}$ M. Zanetti, ${ }^{143}$ V. Zhukova, ${ }^{143}$ B. Dahmes,${ }^{144}$ A. Gude, ${ }^{144}$ S. C. Kao,${ }^{144}$ K. Klapoetke,${ }^{144}$ Y. Kubota, ${ }^{144}$ J. Mans, ${ }^{144}$ N. Pastika, ${ }^{144}$

R. Rusack, ${ }^{144}$ A. Singovsky, ${ }^{144}$ N. Tambe, ${ }^{144}$ J. Turkewitz, ${ }^{144}$ J. G. Acosta, ${ }^{145}$ S. Oliveros, ${ }^{145}$ E. Avdeeva, ${ }^{146}$ K. Bloom, ${ }^{146}$ S. Bose,${ }^{146}$ D. R. Claes, ${ }^{146}$ A. Dominguez, ${ }^{146}$ R. Gonzalez Suarez, ${ }^{146}$ J. Keller, ${ }^{146}$ D. Knowlton, ${ }^{146}$ I. Kravchenko, ${ }^{146}$ J. Lazo-Flores, ${ }^{146}$ F. Meier, ${ }^{146}$ F. Ratnikov, ${ }^{146}$ G. R. Snow, ${ }^{146}$ M. Zvada, ${ }^{146}$ J. Dolen, ${ }^{147}$ A. Godshalk, ${ }^{147}$ I. Iashvili, ${ }^{147}$ A. Kharchilava, ${ }^{147}$ A. Kumar, ${ }^{147}$ S. Rappoccio, ${ }^{147}$ G. Alverson, ${ }^{148}$ E. Barberis, ${ }^{148}$ D. Baumgartel, ${ }^{148}$ M. Chasco, ${ }^{148}$ A. Massironi, ${ }^{148}$ D. M. Morse, ${ }^{148}$ D. Nash, ${ }^{148}$ T. Orimoto, ${ }^{148}$ D. Trocino, ${ }^{148}$ R.-J. Wang, ${ }^{148}$ D. Wood, ${ }^{148}$ J. Zhang, ${ }^{148}$ K. A. Hahn, ${ }^{149}$ A. Kubik, ${ }^{149}$ N. Mucia, ${ }^{149}$ N. Odell, ${ }^{149}$ B. Pollack, ${ }^{149}$ A. Pozdnyakov, ${ }^{149}$ M. Schmitt, ${ }^{149}$ S. Stoynev, ${ }^{149}$ K. Sung, ${ }^{149}$ M. Velasco, ${ }^{149}$ S. Won, ${ }^{149}$ A. Brinkerhoff, ${ }^{150}$ K. M. Chan, ${ }^{150}$ A. Drozdetskiy, ${ }^{150}$ M. Hildreth, ${ }^{150}$ C. Jessop, ${ }^{150}$ D. J. Karmgard, ${ }^{150}$ N. Kellams, ${ }^{150}$ K. Lannon, ${ }^{150}$ S. Lynch, ${ }^{150}$ N. Marinelli, ${ }^{150}$ Y. Musienko, ${ }^{150, \text { ee }}$ T. Pearson,,${ }^{150}$ M. Planer, ${ }^{150}$

R. Ruchti ${ }^{150}$ G. Smith ${ }^{150}$ N. Valls,${ }^{150}$ M. Wayne,${ }^{150}$ M. Wolf ${ }^{150}$ A. Woodard, ${ }^{150}$ L. Antonelli, ${ }^{151}$ J. Brinson, ${ }^{151}$ B. Bylsma, ${ }^{151}$ L. S. Durkin, ${ }^{151}$ S. Flowers, ${ }^{151}$ A. Hart ${ }^{151}$ C. Hill,${ }^{151}$ R. Hughes, ${ }^{151}$ K. Kotov, ${ }^{151}$ T. Y. Ling, ${ }^{151}$ W. Luo, ${ }^{151}$ D. Puigh, ${ }^{151}$ M. Rodenburg, ${ }^{151}$ B. L. Winer, ${ }^{151}$ H. Wolfe, ${ }^{151}$ H. W. Wulsin, ${ }^{151}$ O. Driga, ${ }^{152}$ P. Elmer, ${ }^{152}$ J. Hardenbrook, ${ }^{152}$ P. Hebda ${ }^{152}$ A. Hunt, ${ }^{152}$ S. A. Koay, ${ }^{152}$ P. Lujan, ${ }^{152}$ D. Marlow, ${ }^{152}$ T. Medvedeva,${ }^{152}$ M. Mooney, ${ }^{152}$ J. Olsen, ${ }^{152}$ P. Piroué, ${ }^{152}$ X. Quan, ${ }^{152}$ H. Saka, ${ }^{152}$ D. Stickland, ${ }^{152, c}$ C. Tully, ${ }^{152}$ J. S. Werner, ${ }^{152}$ A. Zuranski, ${ }^{152}$ E. Brownson, ${ }^{153}$ S. Malik, ${ }^{153}$ H. Mendez, ${ }^{153}$ J. E. Ramirez Vargas, ${ }^{153}$ V. E. Barnes, ${ }^{154}$ D. Benedetti, ${ }^{154}$ D. Bortoletto, ${ }^{154}$ M. De Mattia, ${ }^{154}$ L. Gutay, ${ }^{154}$ Z. Hu, ${ }^{154}$ M. K. Jha ${ }^{154}$ M. Jones, ${ }^{154}$ K. Jung, ${ }^{154}$ M. Kress, ${ }^{154}$ N. Leonardo,${ }^{154}$ D. Lopes Pegna, ${ }^{154}$ V. Maroussov, ${ }^{154}$ D. H. Miller, ${ }^{154}$ N. Neumeister, ${ }^{154}$ B. C. Radburn-Smith, ${ }^{154}$ X. Shi, ${ }^{154}$ I. Shipsey, ${ }^{154}$ D. Silvers, ${ }^{154}$ A. Svyatkovskiy, ${ }^{154}$ F. Wang, ${ }^{154}$ W. Xie,${ }^{154}$ L. Xu, ${ }^{154}$ J. Zablocki, ${ }^{154}$ Y. Zheng, ${ }^{154}$ N. Parashar, ${ }^{155}$ J. Stupak, ${ }^{155}$ A. Adair, ${ }^{156}$ B. Akgun, ${ }^{156}$ K. M. Ecklund, ${ }^{156}$ F. J. M. Geurts, ${ }^{156}$ W. Li ${ }^{156}$ B. Michlin, ${ }^{156}$ B. P. Padley, ${ }^{156}$ R. Redjimi, ${ }^{156}$ J. Roberts, ${ }^{156}$ J. Zabel, ${ }^{156}$ B. Betchart, ${ }^{157}$ A. Bodek, ${ }^{157}$ R. Covarelli, ${ }^{157}$ P. de Barbaro, ${ }^{157}$ R. Demina, ${ }^{157}$ Y. Eshaq,${ }^{157}$ T. Ferbel, ${ }^{157}$ A. Garcia-Bellido, ${ }^{157}$ P. Goldenzweig, ${ }^{157}$ J. Han, ${ }^{157}$ A. Harel, ${ }^{157}$ A. Khukhunaishvili, ${ }^{157}$ S. Korjenevski, ${ }^{157}$ G. Petrillo, ${ }^{157}$ D. Vishnevskiy, ${ }^{157}$ R. Ciesielski, ${ }^{158}$ L. Demortier, ${ }^{158}$ K. Goulianos, ${ }^{158}$ C. Mesropian, ${ }^{158}$ S. Arora,${ }^{159}$ A. Barker ${ }^{159}$ J. P. Chou, ${ }^{159}$

C. Contreras-Campana, ${ }^{159}$ E. Contreras-Campana, ${ }^{159}$ D. Duggan, ${ }^{159}$ D. Ferencek, ${ }^{159}$ Y. Gershtein, ${ }^{159}$ R. Gray, ${ }^{159}$ E. Halkiadakis, ${ }^{159}$ D. Hidas, ${ }^{159}$ S. Kaplan, ${ }^{159}$ A. Lath,${ }^{159}$ S. Panwalkar, ${ }^{159}$ M. Park, ${ }^{159}$ R. Patel, ${ }^{159}$ S. Salur, ${ }^{159}$ S. Schnetzer, ${ }^{159}$ S. Somalwar, ${ }^{159}$ R. Stone, ${ }^{159}$ S. Thomas, ${ }^{159}$ P. Thomassen, ${ }^{159}$ M. Walker, ${ }^{159}$ K. Rose, ${ }^{160}$ S. Spanier ${ }^{160}$ A. York, ${ }^{160}$ O. Bouhali, ${ }^{161, \text { eee }}$ A. Castaneda Hernandez, ${ }^{161}$ R. Eusebi, ${ }^{161}$ W. Flanagan, ${ }^{161}$ J. Gilmore, ${ }^{161}$ T. Kamon, ${ }^{161, f f f}$ V. Khotilovich, ${ }^{161}$ V. Krutelyov, ${ }^{161}$ R. Montalvo, ${ }^{161}$ I. Osipenkov, ${ }^{161}$ Y. Pakhotin, ${ }^{161}$ A. Perloff,,${ }^{161}$ J. Roe ${ }^{161}$ A. Rose, ${ }^{161}$ A. Safonov, ${ }^{161}$ I. Suarez, ${ }^{161}$ A. Tatarinov ${ }^{161}$ N. Akchurin, ${ }^{162}$ C. Cowden, ${ }^{162}$ J. Damgov, ${ }^{162}$ C. Dragoiu, ${ }^{162}$ P. R. Dudero, ${ }^{162}$ J. Faulkner, ${ }^{162}$ K. Kovitanggoon, ${ }^{162}$ S. Kunori, ${ }^{162}$ S. W. Lee, ${ }^{162}$ T. Libeiro, ${ }^{162}$ I. Volobouev, ${ }^{162}$ E. Appelt, ${ }^{163}$ A. G. Delannoy, ${ }^{163}$ S. Greene, ${ }^{163}$ A. Gurrola, ${ }^{163}$ W. Johns, ${ }^{163}$ C. Maguire,${ }^{163}$ Y. Mao, ${ }^{163}$ A. Melo, ${ }^{163}$ M. Sharma, ${ }^{163}$ P. Sheldon, ${ }^{163}$ B. Snook, ${ }^{163}$ S. Tuo, ${ }^{163}$ J. Velkovska, ${ }^{163}$ M. W. Arenton, ${ }^{164}$ S. Boutle, ${ }^{164}$ B. Cox,${ }^{164}$ B. Francis, ${ }^{164}$ J. Goodell, ${ }^{164}$ R. Hirosky, ${ }^{164}$ A. Ledovskoy, ${ }^{164}$ H. Li,${ }^{164}$ C. Lin, ${ }^{164}$ C. Neu, ${ }^{164}$ J. Wood, ${ }^{164}$ C. Clarke, ${ }^{165}$ R. Harr, ${ }^{165}$ P. E. Karchin, ${ }^{165}$ C. Kottachchi Kankanamge Don, ${ }^{165}$ P. Lamichhane, ${ }^{165}$ J. Sturdy, ${ }^{165}$ D. A. Belknap, ${ }^{166}$ D. Carlsmith, ${ }^{166}$ M. Cepeda, ${ }^{166}$ S. Dasu, ${ }^{166}$ L. Dodd, ${ }^{166}$ S. Duric, ${ }^{166}$ E. Friis, ${ }^{166}$ R. Hall-Wilton, ${ }^{166}$ M. Herndon,${ }^{166}$ A. Hervé,${ }^{166}$ P. Klabbers, ${ }^{166}$ A. Lanaro, ${ }^{166}$ C. Lazaridis, ${ }^{166}$ A. Levine, ${ }^{166}$ R. Loveless, ${ }^{166}$ A. Mohapatra, ${ }^{166}$ I. Ojalvo, ${ }^{166}$ T. Perry, ${ }^{166}$ G. A. Pierro, ${ }^{166}$ G. Polese, ${ }^{166}$ I. Ross, ${ }^{166}$ T. Sarangi, ${ }^{166}$ A. Savin, ${ }^{166}$ W. H. Smith,${ }^{166}$ D. Taylor, ${ }^{166}$ C. Vuosalo, ${ }^{166}$ and N. Woods ${ }^{166}$

\title{
(CMS Collaboration)
}

\author{
${ }^{1}$ Yerevan Physics Institute, Yerevan, Armenia \\ ${ }^{2}$ Institut für Hochenergiephysik der OeAW, Wien, Austria
}


${ }^{3}$ National Centre for Particle and High Energy Physics, Minsk, Belarus

${ }^{4}$ Universiteit Antwerpen, Antwerpen, Belgium

${ }^{5}$ Vrije Universiteit Brussel, Brussel, Belgium

${ }^{6}$ Université Libre de Bruxelles, Bruxelles, Belgium

${ }^{7}$ Ghent University, Ghent, Belgium

${ }^{8}$ Université Catholique de Louvain, Louvain-la-Neuve, Belgium

${ }^{9}$ Université de Mons, Mons, Belgium

${ }^{10}$ Centro Brasileiro de Pesquisas Fisicas, Rio de Janeiro, Brazil

${ }^{11}$ Universidade do Estado do Rio de Janeiro, Rio de Janeiro, Brazil

${ }^{12 \mathrm{a}}$ Universidade Estadual Paulista, São Paulo, Brazil

${ }^{12 \mathrm{~b}}$ Universidade Federal do ABC, São Paulo, Brazil

${ }^{13}$ Institute for Nuclear Research and Nuclear Energy, Sofia, Bulgaria

${ }^{14}$ University of Sofia, Sofia, Bulgaria

${ }^{15}$ Institute of High Energy Physics, Beijing, China

${ }^{16}$ State Key Laboratory of Nuclear Physics and Technology, Peking University, Beijing, China

${ }^{17}$ Universidad de Los Andes, Bogota, Colombia

${ }^{18}$ University of Split, Faculty of Electrical Engineering, Mechanical Engineering and Naval Architecture, Split, Croatia

${ }^{19}$ University of Split, Faculty of Science, Split, Croatia

${ }^{20}$ Institute Rudjer Boskovic, Zagreb, Croatia

${ }^{21}$ University of Cyprus, Nicosia, Cyprus

${ }^{22}$ Charles University, Prague, Czech Republic

${ }^{23}$ Academy of Scientific Research and Technology of the Arab Republic of Egypt,

Egyptian Network of High Energy Physics, Cairo, Egypt

${ }^{24}$ National Institute of Chemical Physics and Biophysics, Tallinn, Estonia

${ }^{25}$ Department of Physics, University of Helsinki, Helsinki, Finland

${ }^{26}$ Helsinki Institute of Physics, Helsinki, Finland

${ }^{27}$ Lappeenranta University of Technology, Lappeenranta, Finland

${ }^{28}$ DSM/IRFU, CEA/Saclay, Gif-sur-Yvette, France

${ }^{29}$ Laboratoire Leprince-Ringuet, Ecole Polytechnique, IN2P3-CNRS, Palaiseau, France

${ }^{30}$ Institut Pluridisciplinaire Hubert Curien, Université de Strasbourg,

Université de Haute Alsace Mulhouse, CNRS/IN2P3, Strasbourg, France

${ }^{31}$ Centre de Calcul de l'Institut National de Physique Nucleaire et de Physique des Particules, CNRS/IN2P3, Villeurbanne, France

${ }^{32}$ Université de Lyon, Université Claude Bernard Lyon 1, CNRS-IN2P3, Institut de Physique Nucléaire de Lyon, Villeurbanne, France

${ }^{33}$ Institute of High Energy Physics and Informatization, Tbilisi State University, Tbilisi, Georgia

${ }^{34}$ RWTH Aachen University, I. Physikalisches Institut, Aachen, Germany

${ }^{35}$ RWTH Aachen University, III. Physikalisches Institut A, Aachen, Germany

${ }^{36}$ RWTH Aachen University, III. Physikalisches Institut B, Aachen, Germany

${ }^{37}$ Deutsches Elektronen-Synchrotron, Hamburg, Germany

${ }^{38}$ University of Hamburg, Hamburg, Germany

${ }^{39}$ Institut für Experimentelle Kernphysik, Karlsruhe, Germany

${ }^{40}$ Institute of Nuclear and Particle Physics (INPP), NCSR Demokritos, Aghia Paraskevi, Greece

${ }^{41}$ University of Athens, Athens, Greece

${ }^{42}$ University of Ioánnina, Ioánnina, Greece

${ }^{43}$ Wigner Research Centre for Physics, Budapest, Hungary

${ }^{44}$ Institute of Nuclear Research ATOMKI, Debrecen, Hungary

${ }^{45}$ University of Debrecen, Debrecen, Hungary

${ }^{46}$ National Institute of Science Education and Research, Bhubaneswar, India

${ }^{47}$ Panjab University, Chandigarh, India

${ }^{48}$ University of Delhi, Delhi, India

${ }^{49}$ Saha Institute of Nuclear Physics, Kolkata, India

${ }^{50}$ Bhabha Atomic Research Centre, Mumbai, India

${ }^{51}$ Tata Institute of Fundamental Research, Mumbai, India

${ }^{52}$ Institute for Research in Fundamental Sciences (IPM), Tehran, Iran

${ }^{53}$ University College Dublin, Dublin, Ireland

${ }^{54 a}$ INFN Sezione di Bari, Bari, Italy

${ }^{54 \mathrm{~b}}$ Università di Bari, Bari, Italy

${ }^{54}$ Politecnico di Bari, Bari, Italy 
${ }^{55 a}$ INFN Sezione di Bologna, Bologna, Italy ${ }^{55 \mathrm{~b}}$ Università di Bologna, Bologna, Italy

${ }^{56 a}$ INFN Sezione di Catania, Catania, Italy

${ }^{56 \mathrm{~b}}$ Università di Catania, Catania, Italy

${ }^{56}$ CSFNSM, Catania, Italy

${ }^{57 \mathrm{a}}$ INFN Sezione di Firenze, Firenze, Italy

${ }^{57 \mathrm{~b}}$ Università di Firenze, Firenze, Italy

${ }^{58}$ INFN Laboratori Nazionali di Frascati, Frascati, Italy

${ }^{59 a}$ INFN Sezione di Genova, Genova, Italy

${ }^{59 \mathrm{~b}}$ Università di Genova, Genova, Italy

${ }^{60 a}$ INFN Sezione di Milano-Bicocca, Milano, Italy

${ }^{60 \mathrm{~b}}$ Università di Milano-Bicocca, Milano, Italy

${ }^{61 a}$ INFN Sezione di Napoli, Napoli, Italy

${ }^{61 \mathrm{~b}}$ Università di Napoli 'Federico II', Napoli, Italy

${ }^{61 \mathrm{c}}$ Università della Basilicata (Potenza), Napoli, Italy

${ }^{61 \mathrm{~d}}$ Università G. Marconi (Roma), Napoli, Italy

${ }^{62 \mathrm{a}}$ INFN Sezione di Padova, Padova, Italy

${ }^{62 \mathrm{~b}}$ Università di Padova, Padova, Italy

${ }^{62 \mathrm{c}}$ Università di Trento (Trento), Padova, Italy

${ }^{63 a}$ INFN Sezione di Pavia, Pavia, Italy

${ }^{63 \mathrm{~b}}$ Università di Pavia, Pavia, Italy

${ }^{64 a}$ INFN Sezione di Perugia, Perugia, Italy

${ }^{64 \mathrm{~b}}$ Università di Perugia, Perugia, Italy

${ }^{65}$ INFN Sezione di Pisa, Pisa, Italy

${ }^{65 \mathrm{~b}}$ Università di Pisa, Pisa, Italy

${ }^{65 \mathrm{c}}$ Scuola Normale Superiore di Pisa, Pisa, Italy

${ }^{66 a}$ INFN Sezione di Roma, Roma, Italy

${ }^{66 \mathrm{~b}}$ Università di Roma, Roma, Italy

${ }^{67 a}$ INFN Sezione di Torino, Torino, Italy

${ }^{67 b}$ Università di Torino, Torino, Italy

${ }^{67 \mathrm{c}}$ Università del Piemonte Orientale (Novara), Torino, Italy

${ }^{68 a}$ INFN Sezione di Trieste, Trieste, Italy

${ }^{68 \mathrm{~b}}$ Università di Trieste, Trieste, Italy

${ }^{69}$ Kangwon National University, Chunchon, Korea

${ }^{70}$ Kyungpook National University, Daegu, Korea

${ }^{71}$ Chonbuk National University, Jeonju, Korea

${ }^{72}$ Chonnam National University, Institute for Universe and Elementary Particles, Kwangju, Korea

${ }^{73}$ Korea University, Seoul, Korea

${ }^{74}$ Seoul National University, Seoul, Korea

${ }^{75}$ University of Seoul, Seoul, Korea

${ }^{76}$ Sungkyunkwan University, Suwon, Korea

${ }^{77}$ Vilnius University, Vilnius, Lithuania

${ }^{78}$ National Centre for Particle Physics, Universiti Malaya, Kuala Lumpur, Malaysia

${ }^{79}$ Centro de Investigacion y de Estudios Avanzados del IPN, Mexico City, Mexico

${ }^{80}$ Universidad Iberoamericana, Mexico City, Mexico

${ }^{81}$ Benemerita Universidad Autonoma de Puebla, Puebla, Mexico

${ }^{82}$ Universidad Autónoma de San Luis Potosí, San Luis Potosí, Mexico

${ }^{83}$ University of Auckland, Auckland, New Zealand

${ }^{84}$ University of Canterbury, Christchurch, New Zealand

${ }^{85}$ National Centre for Physics, Quaid-I-Azam University, Islamabad, Pakistan

${ }^{86}$ National Centre for Nuclear Research, Swierk, Poland

${ }^{87}$ Institute of Experimental Physics, Faculty of Physics, University of Warsaw, Warsaw, Poland

${ }^{88}$ Laboratório de Instrumentação e Física Experimental de Partículas, Lisboa, Portugal

${ }^{89}$ Joint Institute for Nuclear Research, Dubna, Russia

${ }^{90}$ Petersburg Nuclear Physics Institute, Gatchina (St. Petersburg), Russia

${ }_{91}$ Institute for Nuclear Research, Moscow, Russia

${ }^{92}$ Institute for Theoretical and Experimental Physics, Moscow, Russia

${ }^{93}$ P.N. Lebedev Physical Institute, Moscow, Russia

${ }^{94}$ Skobeltsyn Institute of Nuclear Physics, Lomonosov Moscow State University, Moscow, Russia ${ }^{95}$ State Research Center of Russian Federation, Institute for High Energy Physics, Protvino, Russia 
${ }^{96}$ University of Belgrade, Faculty of Physics and Vinca Institute of Nuclear Sciences, Belgrade, Serbia

${ }^{97}$ Centro de Investigaciones Energéticas Medioambientales y Tecnológicas (CIEMAT), Madrid, Spain

${ }^{98}$ Universidad Autónoma de Madrid, Madrid, Spain

${ }^{99}$ Universidad de Oviedo, Oviedo, Spain

${ }^{100}$ Instituto de Física de Cantabria (IFCA), CSIC-Universidad de Cantabria, Santander, Spain

${ }^{101}$ CERN, European Organization for Nuclear Research, Geneva, Switzerland

${ }^{102}$ Paul Scherrer Institut, Villigen, Switzerland

${ }^{103}$ Institute for Particle Physics, ETH Zurich, Zurich, Switzerland

${ }^{104}$ Universität Zürich, Zurich, Switzerland

${ }^{105}$ National Central University, Chung-Li, Taiwan

${ }^{106}$ National Taiwan University (NTU), Taipei, Taiwan

${ }^{107}$ Chulalongkorn University, Faculty of Science, Department of Physics, Bangkok, Thailand

${ }^{108}$ Cukurova University, Adana, Turkey

${ }^{109}$ Middle East Technical University, Physics Department, Ankara, Turkey

${ }^{110}$ Bogazici University, Istanbul, Turkey

${ }^{111}$ Istanbul Technical University, Istanbul, Turkey

${ }^{112}$ National Scientific Center, Kharkov Institute of Physics and Technology, Kharkov, Ukraine

${ }^{113}$ University of Bristol, Bristol, United Kingdom

${ }^{114}$ Rutherford Appleton Laboratory, Didcot, United Kingdom

${ }^{115}$ Imperial College, London, United Kingdom

${ }^{116}$ Brunel University, Uxbridge, United Kingdom

${ }^{117}$ Baylor University, Waco, Texas 76798, USA

${ }^{118}$ The University of Alabama, Tuscaloosa, Alabama 35487, USA

${ }^{119}$ Boston University, Boston, Massachusetts 02215, USA

${ }^{120}$ Brown University, Providence, Rhode Island 02912, USA

${ }^{121}$ University of California, Davis, Davis, California 95616, USA

${ }^{122}$ University of California, Los Angeles, California 90095, USA

${ }^{123}$ University of California, Riverside, Riverside, California 92521, USA

${ }^{124}$ University of California, San Diego, La Jolla, California 92093, USA

${ }^{125}$ University of California, Santa Barbara, Santa Barbara, California 93106, USA

${ }^{126}$ California Institute of Technology, Pasadena, California 91125, USA

${ }^{127}$ Carnegie Mellon University, Pittsburgh, Pennsylvania 15213, USA

${ }^{128}$ University of Colorado at Boulder, Boulder, Colorado 80309, USA

${ }^{129}$ Cornell University, Ithaca, New York 14853, USA

${ }^{130}$ Fairfield University, Fairfield, Connecticut 06430, USA

${ }^{131}$ Fermi National Accelerator Laboratory, Batavia, Illinois 60510, USA

${ }^{132}$ University of Florida, Gainesville, Florida 32611, USA

${ }^{133}$ Florida International University, Miami, Florida 33199, USA

${ }^{134}$ Florida State University, Tallahassee, Florida 32306, USA

${ }^{135}$ Florida Institute of Technology, Melbourne, Florida 32901, USA

${ }^{136}$ University of Illinois at Chicago (UIC), Chicago, Illinois 60637, USA

${ }^{137}$ The University of Iowa, Iowa 52242, USA

${ }^{138}$ Johns Hopkins University, Baltimore, Maryland 21218, USA

${ }^{139}$ The University of Kansas, Lawrence, Kansas 66045, USA

${ }^{140}$ Kansas State University, Manhattan, Kansas 66506, USA

${ }^{141}$ Lawrence Livermore National Laboratory, Livermore, Michigan 48201, USA

${ }^{142}$ University of Maryland, College Park, Maryland 20742, USA

${ }^{143}$ Massachusetts Institute of Technology, Cambridge, Massachusetts 02139, USA

${ }^{144}$ University of Minnesota, Minneapolis, Minnesota 55455, USA

${ }^{145}$ University of Mississippi, Oxford, Mississippi 38677, USA

${ }^{146}$ University of Nebraska-Lincoln, Lincoln, Nebraska 68588, USA

${ }^{147}$ State University of New York at Buffalo, Buffalo, New York 14260, USA

${ }^{148}$ Northeastern University, Boston, Massachusetts 02115, USA

${ }^{149}$ Northwestern University, Evanston, Illinois 60208, USA

${ }^{150}$ University of Notre Dame, Notre Dame, Indiana 46556, USA

${ }^{151}$ The Ohio State University, Columbus, Ohio 43210, USA

${ }^{152}$ Princeton University, Princeton, New Jersey 08544, USA

${ }^{153}$ University of Puerto Rico, Mayaguez, Puerto Rico 00681, USA

${ }^{154}$ Purdue University, West Lafayette, Indiana 47907, USA

${ }^{155}$ Purdue University Calumet, Hammond, Indiana 46323, USA 


\author{
${ }^{156}$ Rice University, Houston, Texas 77251, USA \\ ${ }^{157}$ University of Rochester, Rochester, New York 14627, USA \\ ${ }^{158}$ The Rockefeller University, New York 10021, USA \\ ${ }^{159}$ Rutgers, The State University of New Jersey, Piscataway, New Jersey 8854, USA \\ ${ }^{160}$ University of Tennessee, Knoxville, Tennessee 37996, USA \\ ${ }^{161}$ Texas A\&M University, College Station, Texas 77843, USA \\ ${ }^{162}$ Texas Tech University, Lubbock, Texas 79409, USA \\ ${ }^{163}$ Vanderbilt University, Nashville, Tennessee 37235, USA \\ ${ }^{164}$ University of Virginia, Charlottesville, Virginia 22904, USA \\ ${ }^{165}$ Wayne State University, Detroit, Michigan 48202, USA \\ ${ }^{166}$ University of Wisconsin, Madison, Wisconsin 53706, USA
}

${ }^{\mathrm{a}}$ Deceased.

${ }^{\mathrm{b}}$ Also at Vienna University of Technology, Vienna, Austria.

${ }^{\mathrm{c}}$ Also at CERN, European Organization for Nuclear Research, Geneva, Switzerland.

${ }^{\mathrm{d}}$ Also at Institut Pluridisciplinaire Hubert Curien, Université de Strasbourg, Université de Haute Alsace Mulhouse, CNRS/IN2P3, Strasbourg, France.

eAlso at National Institute of Chemical Physics and Biophysics, Tallinn, Estonia.

${ }_{\mathrm{f}}^{\mathrm{f}}$ Also at Skobeltsyn Institute of Nuclear Physics, Lomonosov Moscow State University, Moscow, Russia.

${ }^{\mathrm{g}}$ Also at Universidade Estadual de Campinas, Campinas, Brazil.

${ }^{\mathrm{h}}$ Also at Laboratoire Leprince-Ringuet, Ecole Polytechnique, IN2P3-CNRS, Palaiseau, France.

${ }^{\mathrm{i}}$ Also at Joint Institute for Nuclear Research, Dubna, Russia.

${ }^{\mathrm{j}}$ Also at Suez University, Suez, Egypt.

${ }^{\mathrm{k}}$ Also at British University in Egypt, Cairo, Egypt.

${ }^{1}$ Also at Fayoum University, El-Fayoum, Egypt.

${ }^{\mathrm{m}}$ Also at Ain Shams University, Cairo, Egypt.

${ }^{\mathrm{n}}$ Also at Sultan Qaboos University, Muscat, Oman.

${ }^{\circ}$ Also at Université de Haute Alsace, Mulhouse, France.

${ }^{\mathrm{p}}$ Also at Brandenburg University of Technology, Cottbus, Germany.

${ }^{\mathrm{q}}$ Also at Institute of Nuclear Research ATOMKI, Debrecen, Hungary.

${ }^{\mathrm{r}}$ Also at Eötvös Loránd University, Budapest, Hungary.

${ }^{\mathrm{s}}$ Also at University of Debrecen, Debrecen, Hungary.

${ }^{\mathrm{t}}$ Also at University of Visva-Bharati, Santiniketan, India.

${ }^{u}$ Also at King Abdulaziz University, Jeddah, Saudi Arabia.

${ }^{v}$ Also at University of Ruhuna, Matara, Sri Lanka.

${ }^{w}$ Also at Isfahan University of Technology, Isfahan, Iran.

${ }^{\mathrm{x}}$ Also at University of Tehran, Department of Engineering Science, Tehran, Iran.

${ }^{y}$ Also at Plasma Physics Research Center, Science and Research Branch, Islamic Azad University, Tehran, Iran.

${ }^{\mathrm{z}}$ Also at Laboratori Nazionali di Legnaro dell'INFN, Legnaro, Italy.

${ }^{\text {aa } A l s o ~ a t ~ U n i v e r s i t a ̀ ~ d e g l i ~ S t u d i ~ d i ~ S i e n a, ~ S i e n a, ~ I t a l y . ~}$

${ }^{\mathrm{bb}}$ Also at Centre National de la Recherche Scientifique (CNRS) - IN2P3, Paris, France.

${ }^{\mathrm{cc}}$ Also at Purdue University, West Lafayette, USA.

${ }^{\mathrm{dd}}$ Also at Universidad Michoacana de San Nicolas de Hidalgo, Morelia, Mexico.

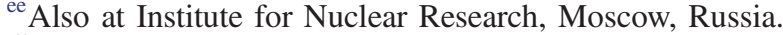

${ }^{\mathrm{ff}}$ Also at St. Petersburg State Polytechnical University, St. Petersburg, Russia.

${ }^{g g}$ Also at California Institute of Technology, Pasadena, USA.

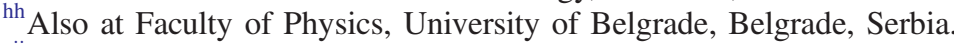

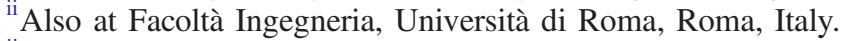

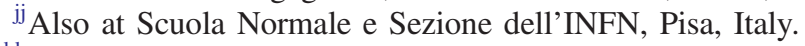

${ }^{\mathrm{kk}}$ Also at University of Athens, Athens, Greece.

${ }^{11}$ Also at Paul Scherrer Institut, Villigen, Switzerland.

${ }^{\mathrm{mm}}$ Also at Institute for Theoretical and Experimental Physics, Moscow, Russia.

${ }^{n n}$ Also at Albert Einstein Center for Fundamental Physics, Bern, Switzerland.

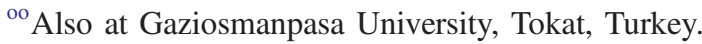

${ }^{\mathrm{pp}}$ Also at Adiyaman University, Adiyaman, Turkey.

${ }^{\mathrm{qq}}$ Also at Cag University, Mersin, Turkey.

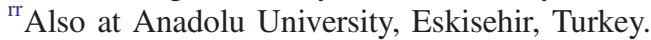

${ }^{\text {ss }}$ Also at Ozyegin University, Istanbul, Turkey.

${ }^{t t}$ Also at Izmir Institute of Technology, Izmir, Turkey.

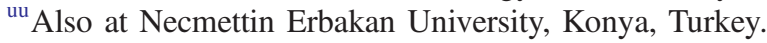


${ }^{\mathrm{vv}}$ Also at Mimar Sinan University, Istanbul, Istanbul, Turkey.

${ }^{w w}$ Also at Marmara University, Istanbul, Turkey.

${ }^{\mathrm{xx}}$ Also at Kafkas University, Kars, Turkey.

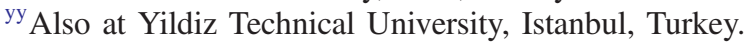

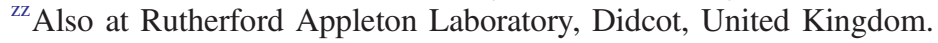

${ }^{\text {aaa }}$ Also at School of Physics and Astronomy, University of Southampton, Southampton, United Kingdom.

${ }^{b b b}$ Also at University of Belgrade, Faculty of Physics and Vinca Institute of Nuclear Sciences, Belgrade, Serbia.

${ }^{c c c}$ Also at Argonne National Laboratory, Argonne, USA.

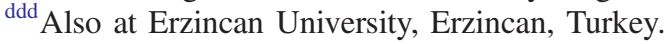

${ }^{e e e}$ Also at Texas A\&M University at Qatar, Doha, Qatar.

${ }^{\mathrm{fff}}$ Also at Kyungpook National University, Daegu, Korea. 